\title{
ON ASYMPTOTIC STABILITY OF SOLITARY WAVES FOR NONLINEAR SCHRÖDINGER EQUATIONS
}

\section{STABILITÉ ASYMPTOTIQUE DES ONDES SOLITAIRES POUR LES ÉQUATIONS DE SCHRÖDINGER NONLINÉAIRES}

\author{
Vladimir S. BUSLAEV ${ }^{\text {a }}$, Catherine SULEM ${ }^{\text {b,* }}$ \\ ${ }^{a}$ Institute for Physics, St. Petersburg State University, St. Petersburg-Petrodvorets, 198904, Russia \\ ${ }^{\mathrm{b}}$ Department of Mathematics, University of Toronto, Toronto, M5S 3G3 Canada
}

Received 31 October 2001, revised 1 April 2002

\begin{abstract}
We study the long-time behavior of solutions of the nonlinear Schrödinger equation in one space dimension for initial conditions in a small neighborhood of a stable solitary wave. Under some hypothesis on the structure of the spectrum of the linearized operator, we prove that, asymptotically in time, the solution decomposes into a solitary wave with slightly modified parameters and a dispersive part described by the free Schrödinger equation. We explicitly calculate the time behavior of the correction.

(C) 2003 L'Association Publications de l'Institut Henri Poincaré. Published by Elsevier B.V. All rights reserved
\end{abstract}

MSC: 35Q55; 37K40

Keywords: Nonlinear Schrödinger equations; Solitary waves; Asymptotic stability

RÉSUMÉ. - On étudie le comportement aux temps longs des solutions de l'équation de Schrödinger nonlinéaire unidimensionnelle pour des conditions initiales proches d'une onde solitaire stable. Moyennant des hypothèses sur la structure du spectre de l'opérateur linearisé autour du soliton, on montre qu'asymptotiquement en temps, la solution se décompose en la somme d'une onde solitaire avec des paramètres faiblement modifiés et d'une composante dispersive, solution de l'équation de Schrödinger libre. On calcule explicitement le comportement en temps des termes correctifs.

(C) 2003 L'Association Publications de l'Institut Henri Poincaré. Published by Elsevier B.V. All rights reserved

\footnotetext{
* Corresponding author.

E-mail addresses: buslaev@ mph.phys.spbu.ru (V.S. Buslaev), sulem@ math.toronto.edu (C. Sulem).
} 


\section{Introduction}

This article deals with the scattering theory of the Nonlinear Schrödinger equation in one space dimension

$$
\begin{gathered}
i \psi_{t}=-\psi_{x x}+F\left(|\psi|^{2}\right) \psi, \quad x \in \mathbb{R}, \\
\psi(x, 0)=\psi_{0}(x)
\end{gathered}
$$

where $\psi(x, t)$ is complex-valued function. We suppose that it possesses solitary wave solutions of the form

$$
\psi(x, t)=\mathrm{e}^{i \omega t} \varphi(x, \omega)
$$

where $\varphi$ is the positive solution of the equation

$$
\varphi^{\prime \prime}-\omega \varphi-F\left(\varphi^{2}\right) \varphi=0
$$

vanishing exponentially at infinity. The problem of stability of solitary waves for nonlinear dispersive equations has been the object of numerous works $[1,8,16]$.

We suppose that (1.0.1) possesses stable solitary wave solutions and we investigate their asymptotic stability, that is the long-time behavior of solutions whose initial conditions are close to a stable solitary wave. In the case of integrable nonlinear equations (such as Korteweg-de Vries equation, cubic Schrödinger equation, BenjaminOno equation...), the inverse scattering method, under certain conditions, provides an asymptotic decomposition of the solution into a sum of solitary waves and a dispersive component. In this paper, we deal with non-integrable equations and the approach is completely different and local.

Our method, initiated in [2], is based on the spectral decomposition of the solution on the eigenspaces associated to the discrete and continuous spectrum of the linearized operator near the solitary wave. To present our result, we need to introduce some notations and state the hypothesis. It is convenient to rewrite the nonlinear Schrödinger equation in the vectorial form

$$
\begin{gathered}
j \psi_{t}=-\psi_{x x}+F\left(|\psi|^{2}\right) \psi, \\
\psi(x, 0)=\psi_{0}(x)
\end{gathered}
$$

where $j=\left(\begin{array}{cc}0 & -1 \\ 1 & 0\end{array}\right), \psi=\left(\begin{array}{l}\psi_{1} \\ \psi_{2}\end{array}\right), \psi_{1}=\operatorname{Re} \psi, \psi_{2}=\operatorname{Im} \psi$.

For technical reasons, we restrict ourselves to even solutions.

Assumption (NL). - We suppose that the nonlinearity $F(s)$ is a $C^{r}$-function of $s \geqslant 0$, such that $s=0$ is a root of multiplicity $r$ with $r \geqslant 4$, and that for $s>1$, it satisfies the lower estimate

$$
F(s) \geqslant-F_{1} s^{q}, \quad \text { with } F_{1}>0, q<2 .
$$

Assumption (1.0.7) ensures that for an initial condition $\psi_{0}$ in the Sobolev space $H^{1}(\mathbb{R})$, the solution $\psi(x, t)$ exists for all time as a continuous function of $t$ with values in $H^{1}(\mathbb{R})$. In addition, if initially $x \psi_{0} \in L^{2}(\mathbb{R})$, then $x \psi(x, t)$ remains in $L^{2}(\mathbb{R})$ for all time [6]. 
Assumption (SL). - Further assumption is made in terms of

$$
U(\varphi)=-\frac{\omega}{2} \varphi^{2}-\frac{1}{2} \int_{0}^{\varphi^{2}} F(s) d s .
$$

We assume that, for all $\omega$ in an interval centered at some $\omega_{0}$, the mapping $\varphi \rightarrow$ $U(\varphi)$ has a positive root and the smallest positive root $\varphi_{0}$ is simple. We also assume $U^{\prime}\left(\varphi_{0}\right) \neq 0$.

Under this assumption there exists a unique, even solution $\varphi(x, \omega)$ of $\varphi_{x x}=-U_{\varphi}$, decreasing like $A(\omega) \mathrm{e}^{-\sqrt{\omega} x}$ as $x \rightarrow+\infty$. Eq. (1.0.5) has solutions in the form of solitary waves $\mathrm{e}^{j \omega t} \phi, \phi=\left(\begin{array}{c}\varphi(x, \omega) \\ 0\end{array}\right)$.

The linearized operator near the solitary wave $\mathrm{e}^{j \omega t} \phi$ is

$$
B u=-\partial_{x x} u+\omega u+F\left(|\phi|^{2}\right) u+2 F^{\prime}\left(|\phi|^{2}\right)(u, \phi) \phi,
$$

where $(.,$.$) denotes the usual scalar product in \mathbb{C}^{2}$ defined by $(u, v)=u_{1} \bar{v}_{1}+u_{2} \bar{v}_{2}$.

Let $C=j^{-1} B=j^{-1}\left(-\partial_{x x}+\omega\right)+V$. In general, the spectrum of $C$ is located on the real and imaginary axis. The continuous spectrum is located on the two half axis $(-i \infty,-i \omega] \cup[i \omega, i \infty)$. Because the potential decreases exponentially fast at infinity, the discrete spectrum is composed of a finite number of eigenvalues. The corresponding invariant spaces are of finite dimension. The point 0 belongs to the discrete spectrum and the dimension of its invariant subspace is at least 2. Recall that we restrict the operator $C$ to even solutions. We assume more specific conditions:

Assumption (SP). - There is no real eigenvalue except $\lambda=0$, and the invariant subspace associated to the eigenvalue $\lambda=0$ is of dimension exactly 2 . In addition, there are 2 simple eigenvalues $\pm i \mu$, which satisfy the property $2 \mu>\omega$. Their corresponding eigenspaces are of dimension 1 . We assume the generic condition that the edges of the continuous spectrum $\pm i \omega$ are not resonances, or equivalently, that there are no solutions, bounded at infinity (virtual levels), nor bound states of $C u= \pm i \omega u$. We also assume that there are no embedded eigenvalues in the continuous spectrum.

If Assumptions (SL) and (SP) are true for a fixed value $\omega_{0}$, they are also true for values of $\omega$ in a small interval centered at $\omega_{0}$. A detailed analysis of the spectral theory of the operator $C$ was developed in [2]. A key point in the analysis of the non self-adjoint operator $C$ is that the coefficients of the matrix-potential $V$ decrease exponentially fast at infinity. The hypothesis on the spectrum of $C(\omega)$ ensures orbital stability of solitary waves.

We consider initial conditions $\psi_{0}$ in the form:

$$
\psi_{0}(x)=\phi\left(x, \omega_{0}\right)+\left(z_{0} u\left(x, \omega_{0}\right)+\bar{z}_{0} u^{*}\left(x, \omega_{0}\right)\right)+f_{0}(x)
$$

where $u\left(x, \omega_{0}\right)$ and $u^{*}\left(x, \omega_{0}\right)$ are the eigenvectors of $C\left(\omega_{0}\right)$ associated to the eigenvalues $\pm i \mu\left(\omega_{0}\right)$, and $f_{0}$ belongs to the eigenspace associated to the continuous spectrum of $C\left(\omega_{0}\right)$. We also assume a non-degeneracy condition. Let $\langle\cdot, \cdot\rangle$ denote the scalar product 
in $L^{2}$ of $\mathbb{C}^{2}$-valued functions: $\langle u, v\rangle=\int_{\mathbb{R}}(u, v) d x$, and $E_{2}[f, f]$ be the quadratic terms coming from the Taylor expansion of the nonlinearity:

$$
E_{2}[f, f]=F^{\prime}\left(|\phi|^{2}\right)|f|^{2} \phi+2 F^{\prime \prime}\left(|\phi|^{2}\right)(\phi, f)^{2} \phi+2 F^{\prime}\left(|\phi|^{2}\right)(\phi, f) f .
$$

The condition has the form

$$
\left\langle E_{2}[u, u], u\left(2 i \mu_{0}\right)\right\rangle \neq 0
$$

where $u\left(2 i \mu_{0}\right)$ is the eigenfunction associated to $\lambda=2 i \mu_{0}=2 i \mu\left(\omega_{0}\right)$. This condition expresses that the interaction of the term of double frequency $2 \mu_{0}$ generated by the nonlinearity with the continuous spectrum is nontrivial and is sometimes referred to as a nonlinear version of the Fermi Golden rule.

Let $\left|z_{0}\right|=\varepsilon^{1 / 2}$ and $N \equiv\left\|f_{0}\right\|_{H^{1}}+\left\|\left(1+x^{2}\right) f_{0}\right\|_{2} \leqslant c \varepsilon^{3 / 2}$ where $c$ is a constant. For $\varepsilon$ sufficiently small, we construct a solution in the form

$$
\psi(x, t)=\mathrm{e}^{j\left(\int_{0}^{t} \omega(s) d s+\gamma(t)\right)}(\phi(x, \omega(t))+w(x, t)+f(x, t)),
$$

where $w(x, t)=z(t) u(x, \omega(t))+\bar{z}(t) u^{*}(x, \omega(t))$, and $f(x, t)$ belongs to the subspace associated to the continuous spectrum of $C(\omega(t))$. The dependency on $t$ of $\omega$ and $\gamma$ is defined by the structure of the solution. We show that, as $t \rightarrow+\infty, \omega(t) \rightarrow \omega_{+}$, and

$$
\psi(x, t)=\mathrm{e}^{j \Phi_{+}(t)}\left[\phi\left(x, \omega_{+}\right)+z_{+}(t) u\left(x, \omega_{+}\right)+\bar{z}_{+}(t) u^{*}\left(x, \omega_{+}\right)\right]+\mathrm{e}^{j^{-1} L t} h_{+}+\mathrm{o}(1)
$$

in $L^{2}$, where o(1) is taken with respect to the variable $t$,

$$
\Phi_{+}(t)=\omega_{+} t+c_{+} \log \left(1+k_{+} \varepsilon t\right)+\gamma_{+},
$$

$\omega_{+}, c_{+}, k_{+}$and $\gamma_{+}$are constants, $k_{+}>0, L=-\frac{\partial^{2}}{\partial x^{2}}$,

$$
z_{+}(t)=\varepsilon^{1 / 2} \frac{\zeta_{+} \mathrm{e}^{i \mu_{+} t}}{\left(1+k_{+} \varepsilon t\right)^{1 / 2-i \delta}}
$$

$\mu_{+}=\mu\left(\omega_{+}\right), \delta$ a real constant and $\zeta_{+}=\mathrm{O}(1)$ as $\varepsilon \rightarrow 0$, and $h_{+} \in L^{2}$, independent of $t$. Asymptotically in $t, f(x, t)$ reduces to a purely dispersive wave. We have as $t \rightarrow \infty$,

$$
\|f\|_{2}=\left\|\mathrm{e}^{j^{-1} L t} h_{+}\right\|_{2}+\mathrm{o}(1) .
$$

If $x$ is bounded (i.e., when $f$ is estimated in a norm with a decreasing weight) then $f=\mathrm{O}\left(\left(\frac{\varepsilon}{1+\varepsilon t}\right)^{3 / 2}\right)$, while for large $x,\left(f\right.$ is then estimated in $L^{\infty}$-norm) $f=\mathrm{O}\left(\left(\frac{\varepsilon}{1+\varepsilon t}\right)^{1 / 2}\right)$. Furthermore, we have, for the conservation of mass

$$
\|\psi(., t)\|_{2}^{2}=\left\|\varphi_{\omega_{+}}\right\|_{2}^{2}+\left\|h_{+}\right\|_{2}^{2} .
$$

An analogous formula for the energy is also true. 
The result shows that in the neighborhood of a stable soliton state, the system is equivalent to the direct sum of two systems: the first one is a Hamiltonian system with one degree of freedom and the second is the free Schrödinger equation. The linear dispersion works like dissipation, leading to a locally vanishing radiation part and eventually to an independent behavior of the localized soliton and the spreading radiative term. Under the Fermi Golden rule assumption, the interaction between higher harmonics of the soliton and the radiative field remains always nontrivial.

The case where the discrete spectrum is reduced to $\lambda=0$ was studied in [2]. In a subsequent paper, the authors [3] proved the asymptotic stability of solitary waves when the operator $C\left(\omega_{0}\right)$ satisfies the spectral properties (SP). Following their ideas, we develop a more transparent and explicit calculation of the splitting of motions, and give a detailed description and explicit formulas for the correction terms. In particular, we calculate the period of oscillations of the phase as well as of the amplitude of the solution. An heuristic analysis of the phenomenon of amplitude oscillations was developed in [11].

The question of asymptotic stability has been investigated for various related problems. Soffer and Weinstein [12] considered the nonlinear Schrödinger equation with a potential term

$$
i \psi_{t}+\Delta \psi=\left(V(x)+\lambda|\psi|^{m-1}\right) \psi
$$

for $x \in \mathbb{R}^{d}$, and $1<m<(d+2) /(d-2)$. Under the assumptions that $V(x)$ decays fast enough at infinity and that the operator $-\Delta+V$ has exactly one bound state (isolated eigenvalue) in $L^{2}\left(\mathbb{R}^{2}\right)$, with strictly negative eigenvalue $E_{*}$, they proved that for a class of initial conditions, the solution of (1.0.19) is given by $\psi=\mathrm{e}^{-i \Theta(t)} \varphi_{E(t)}+f(t)$, $\Theta=\int_{0}^{t} E(s) d s-\gamma(t)$, where $\varphi_{E}$ is a spatially localized solitary wave and $f$ a purely dispersive wave. As $t \rightarrow \pm \infty, E(t) \rightarrow E_{ \pm}$and $\gamma(t) \rightarrow \gamma_{ \pm}$. The case where the operator $-\Delta+V$ has 2 bound states was investigated recently by Tsai and Yau [17] using ideas that Soffer and Weinstein [13] developed in the context of resonance solutions of the nonlinear Klein-Gordon equation.

The scattering theory of the Nonlinear Schrödinger equation

$$
i \psi_{t}+\Delta \psi-\varepsilon|\psi|^{l} \psi=0, \quad l>2, \varepsilon>0,
$$

with respect to the free Schrödinger equation

$$
i \psi_{t}+\Delta \psi=0
$$

was studied by Ginibre and Velo [6], Strauss [14], McKean and Shatah [9]. This case corresponds to the absence of bound states.

Deift and Zhou [5] considered a perturbation

$$
i \psi_{t}+\psi_{x x}-2|\psi|^{2} \psi-\varepsilon|\psi|^{l} \psi=0, \quad l>2, \varepsilon>0,
$$

of the defocusing one-dimensional cubic Schrödinger equation

$$
i \psi_{t}+\psi_{x x}-2|\psi|^{2} \psi=0
$$


Since (1.0.23) is completely integrable, they viewed (1.0.22) as a perturbation of an infinite dimensional integrable system on the line. They proved that as $t \rightarrow \infty$, solutions of (1.0.22) behave like solutions of (1.0.23) and that the long-time behavior is universal for a large class of initial data.

Cuccagna [4] extended the analysis of [2] to the case of spatial dimension larger or equal to 3. The method of decomposition of motion was recently used to prove rigorously the blow-up properties of the nonlinear Schrödinger equation with critical power nonlinearity in one space dimension [10] (see [15] for a review of the properties of blowing-up solutions).

The paper is organized as follows. In Section 2, we recall basic facts about the decomposition of motions and the linearized operator and we derive a system of equations for the various components of the solution in the form

$$
\begin{aligned}
& \dot{\omega}=\bar{\Omega}(\omega, z, f), \quad \dot{\gamma}=\bar{\Gamma}(\omega, z, f), \\
& \dot{z}=i \mu z+\bar{Z}(\omega, z, f), \quad \dot{f}=C(\omega) f+\bar{F}(\omega, z, f) .
\end{aligned}
$$

In Section 3, we calculate the leading terms in the equations, and estimate the remainders. In Section 4, we transform the evolution equations to a simpler, canonical form using the idea of normal coordinates, with the purpose of keeping unchanged the estimates for the remainders. In Section 5, we introduce the notion of majorants defined in terms of norms of $\omega(t), \gamma(t), z(t)$, and $f(x, t)$, with appropriate time dependent weights in a fixed interval of time $[0, T]$ and establish uniform bounds independent of $T$, for initial conditions sufficiently close to a solitary wave. In Section 6 , we find the precise long time behavior of the various components of the solution.

Notice that in general, it is not easy in practice to check the spectral properties of a given operator. For the purpose of examples, and for simplicity, let us restrict to a polynomial nonlinearity. The condition of orbital stability can be expressed in terms of sign of the derivative of the $L^{2}$-norm of the solitary wave with respect to $\omega$. In the case of a power law $F(s)=-s^{p}$ we have $\left\|\varphi_{\omega}\right\|_{2}^{2}=\omega^{2 / p-1}\left\|\varphi_{1}\right\|_{2}^{2}$, and the condition for orbital stability is that $\frac{d}{d \omega}\left\|\varphi_{\omega}\right\|_{2}^{2}>0$ or equivalently $p<2$. The case $p=1$ is the integrable case, with a discrete spectrum of the linearized operator reduced to $\lambda=0$ and the boundary of the continuous spectrum $\pm i \omega$ being resonances. For $1<p<2$, the linearized operator has the required spectrum: one eigenvalue between 0 and the boundary of the continuous spectrum, and no resonances. If $p$ is close to 1 , the eigenvalue is close to the boundary of the continuous spectrum. To satisfy that the nonlinearity has a root of high multiplicity at 0 (condition (NL)), one can perturbe slightly the nonlinearity $F(s)$ only near the origin. The soliton will change slightly and it follows from the general theory of linear ordinary differential operators that the general structure of the spectrum will not be modified and the eigenvalue remains close to its original location. Now we have to take care of the nonlinearity $F(s)$ at infinity. If we change the nonlinearity only for values that are larger than the maximum value of the soliton solution, this does not affect the structure of the soliton and therefore, the spectral properties of the linearized operator. Thus, by this modification of the nonlinearity, we can satisfy the conditions on $F(s)$ for large $s$. As for embedded eigenvalues, if they exist, a small perturbation of the nonlinearity will eliminate them. 
Grikurov [7] investigated numerically the spectral properties of the linearized operator near a solitary wave of the NLS equation with a saturated nonlinearity:

$$
i u_{t}+u_{x x}+|u|^{2 p} u-\alpha|u|^{2 q} u=0,
$$

with $p=3, q=6$, for various small values of the coefficient $\alpha$. He observed that the point spectrum is composed, in addition to the eigenvalue $\lambda=0$, of two opposite real eigenvalues when $\alpha$ is smaller than a specific value $\alpha_{*}$, while for $\alpha>\alpha_{*}$, it is composed of two complex conjugate imaginary eigenvalues.

We conclude this part by listing some open problems that can be seen as natural extensions to this work. One could replace the hypothesis $2 \mu>\omega$ of Assumption (SP) by $n \mu>\omega$, and also allow more than one pair of eigenvalues $\pm i \mu$. One could also drop the restriction to even solutions and consider general solutions. This would lead to two additional equations to (1.0.24)-(1.0.25) for the center and the velocity of the solitary wave. A more difficult problem is to allow the presence of resonances at the edge of the continuous spectrum.

Notations. - All integrals are taken over $\mathbb{R}$ unless indicated otherwise. Norms in $L^{p}$ spaces are denoted $\|\cdot\|_{p}$ and $\|f\|_{\rho}=\|\rho f\|_{2}$ denotes the weighted norm in $L^{2}(\rho)$ with the decreasing weight $\rho(x)=\left(1+x^{2}\right)^{-\alpha}$, where $\alpha>0$ will be fixed later.

\section{Decomposition of motion}

\subsection{The soliton and the linearization near the soliton}

The linearized operator near the solitary wave $\mathrm{e}^{j \omega t} \phi, \phi=\left(\begin{array}{l}\varphi \\ 0\end{array}\right)$, where $\varphi$ is the positive solution, decreasing like $A(\omega) \mathrm{e}^{-\sqrt{\omega}|x|}$ at infinity, of

$$
\left(-\frac{d^{2}}{d x^{2}}+\omega\right) \varphi+F\left(\varphi^{2}\right) \varphi=0
$$

is

$$
B u=\left(-\frac{\partial^{2}}{\partial x^{2}}+\omega+F\left(|\phi|^{2}\right)\right) u+2 F^{\prime}\left(|\phi|^{2}\right)(u, \phi) \phi
$$

Equivalently,

$$
B u=\left(\begin{array}{c}
\mathcal{D}_{1} u_{1} \\
\mathcal{D}_{2} u_{2}
\end{array}\right),
$$

where $\mathcal{D}_{1}=-\frac{\partial^{2}}{\partial x^{2}}+\omega+F\left(|\phi|^{2}\right)+2 F^{\prime}\left(|\phi|^{2}\right)|\phi|^{2}$, and $\mathcal{D}_{2}=-\frac{\partial^{2}}{\partial x^{2}}+\omega+F\left(|\phi|^{2}\right)$. The operator

$$
C=j^{-1} B=j^{-1}\left(-\frac{\partial^{2}}{\partial x^{2}}+\omega\right)+V
$$

is defined as

$$
C u=\left(\begin{array}{c}
\mathcal{D}_{2} u_{2} \\
-\mathcal{D}_{1} u_{1}
\end{array}\right)
$$


We assume that the spectrum of $C$ has the structure described in Assumption (SP). We denote by $X_{0}$ the invariant space associated to $\lambda=0$, and $X_{1}$ and $X_{c}$, the eigenspaces associated to $\lambda= \pm i \mu$, and the continuous spectrum respectively. It is also useful to denote $X_{d}=X_{0}+X_{1}$. Note that, if $C^{*}$ is the adjoint operator of $C$, we have $C^{*} j=-j C$, while $-C \sigma_{3}=\sigma_{3} C$, where $\sigma_{3}=\left(\begin{array}{cc}1 & 0 \\ 0 & -1\end{array}\right)$.

Define $\chi_{0}=j \phi$. We have $C \chi_{0}=0$. In addition, $\chi_{1}=\frac{\partial \phi}{\partial \omega}=\phi_{\omega}$ satisfies $C \chi_{1}=\chi_{0}$. The invariant space $X_{0}$ associated to $\lambda=0$ is spanned by $\chi_{0}$ and $\chi_{1}$. The spectral projection $P_{0}$ of a vector valued function $f$ on $X_{0}$ is defined by

$$
P_{0} f=\frac{1}{\left\langle\phi, \phi_{\omega}\right\rangle}\left(\left\langle f, j \phi_{\omega}\right\rangle j \phi+\langle f, \phi\rangle \phi_{\omega}\right) .
$$

Let $u=\left(\begin{array}{l}u_{1} \\ u_{2}\end{array}\right)$ be the eigenvector of $C$ associated to $i \mu$. We have

$$
i \mu u_{1}=\mathcal{D}_{2} u_{2}, \quad \text { and } \quad i \mu u_{2}=-\mathcal{D}_{1} u_{1} .
$$

This implies that $\mathcal{D}_{2} \mathcal{D}_{1} u_{1}=\mu^{2} u_{1}$. Since $\mathcal{D}_{2} \mathcal{D}_{1}$ is a real operator, it is possible to choose the function $u_{1}(x)$ real. From (2.1.5), we see that $u_{2}$ is then purely imaginary. This will be our choice throughout this paper. We denote by $u^{*}=\left(\begin{array}{c}u_{1} \\ -u_{2}\end{array}\right)$, the eigenvector associated to $-i \mu$. The spectral projection $P_{1}$ of an arbitrary vector-valued function $f$ on $X_{1}$ is

$$
P_{1} f=\frac{\langle f, j u\rangle}{\langle u, j u\rangle} u+\frac{\left\langle f, j u^{*}\right\rangle}{\left\langle u^{*}, j u^{*}\right\rangle} u^{*} .
$$

Finally, the spectral projection $P_{c}$ on $X_{c}$ is $P_{c}=I-P_{d}=I-P_{0}-P_{1}$. It is easy to see that the projection operators satisfy the property

$$
j P_{0}=P_{0}^{*} j, \quad j P_{1}=P_{1}^{*} j, \quad j P_{c}=P_{c}^{*} j .
$$

Denote by $u(i \lambda)$ and $u^{*}(i \lambda)$ the solutions of

$$
C u=i \lambda u, \quad C u^{*}=-i \lambda u^{*},
$$

where $\lambda>\omega$. For $f \in X_{c}$, we have the spectral representation

$$
f=\int_{\omega}^{\infty} d \lambda\left(\theta_{+}(\lambda)\langle f, j u(i \lambda)\rangle u(i \lambda)+\theta_{-}(\lambda)\left\langle f, j u^{*}(i \lambda)\right\rangle u^{*}(i \lambda)\right) .
$$

By orthogonality

$$
\begin{aligned}
\theta_{+}(\lambda)\left\langle u(i \lambda), j u\left(i \lambda^{\prime}\right)\right\rangle & =\delta\left(\lambda-\lambda^{\prime}\right), \\
\theta_{-}(\lambda)\left\langle u^{*}(i \lambda), j u^{*}\left(i \lambda^{\prime}\right)\right\rangle & =\delta\left(\lambda-\lambda^{\prime}\right)
\end{aligned}
$$

and $\theta_{+}(\lambda)=\theta_{-}(\lambda)$. In the limit $|x| \rightarrow \infty$, up to terms exponentially decreasing at infinity, we have, for $\lambda>\omega$,

$$
u_{1}(i \lambda) \sim N(\lambda) \cos (\sqrt{\lambda-\omega}|x|-\vartheta(\lambda))
$$


where $N(\lambda)$ is a real normalization constant, and $\vartheta(\lambda)$ a phase factor. For large $x, u_{2}(i \lambda) \sim i u_{1}(i \lambda)$. The smallest exponential rate $|\beta|$ of the decaying remainders $\exp (-|\beta||x|)$ is equal to $(2 \omega)^{1 / 2}$.

Calculating the improper scalar product $\langle u(i \lambda), j u(i \lambda)\rangle$ in this limit, one gets

$$
\theta_{-}(\lambda)=\theta_{+}(\lambda)=\frac{1}{i} \theta(\lambda), \quad \text { with } \theta(\lambda)=\frac{1}{2 \pi N^{2}(\lambda) \sqrt{\lambda-\omega}},
$$

and the spectral decomposition (2.1.9) rewrites

$$
P_{c} f=\frac{1}{i} \int_{\omega}^{\infty} \theta(\lambda) d \lambda\left(\langle f, j u(i \lambda)\rangle u(i \lambda)+\left\langle f, j u^{*}(i \lambda)\right\rangle u^{*}(i \lambda)\right) .
$$

Denoting by $P_{+}$and $P_{-}$the projection operators on the spectral space associated to the positive and negative part of the continuous spectrum respectively, we have:

Proposition 2.1 [3]. - For $f \in X_{c}$,

$$
\left\|\left(1+x^{2}\right)\left(P_{c} j^{-1} f-i\left(P_{+}-P_{-}\right) f\right)\right\|_{2} \leqslant K(\omega)\|f\|_{\rho},
$$

where $\rho(x)=\left(1+x^{2}\right)^{-\alpha}, \alpha>0$ arbitrary, and $K$ is a constant depending on $\omega$.

In Appendix A, we recall several properties of the spectral resolution (2.1.13) and give some remarks on the above proposition.

\subsection{The dynamical equations}

We look for a solution in the form

$$
\psi(x, t)=\mathrm{e}^{j\left(\int_{0}^{t} \omega(s) d s+\gamma(t)\right)} \Psi(x, t),
$$

with

$$
\Psi=\phi+\chi .
$$

In (2.2.2), $\phi=(\varphi, 0)$, with $\varphi$ solution of

$$
\left(-\frac{d^{2}}{d x^{2}}+\omega(t)\right) \varphi+F\left(\varphi^{2}\right) \varphi=0
$$

and $\chi=w(x, t)+f(x, t)$ where $w=z(t) u+\bar{z}(t) u^{*} \in X_{1}$ and $f \in X_{c}$. Notice that $u$ and $u^{*}$ depend on $\omega$ and thus on $t$. Substituting (2.2.2) into (1.0.1), one gets

$$
-\dot{\gamma} \Psi+j \dot{\Psi}=B \chi+E[\chi],
$$

where $B$ is the linearized operator defined in (2.1.2) and $E[\chi]$ contains all the remainder terms which are at least quadratic. Defining $Q[\chi]=j^{-1} E[\chi],(2.2 .4)$ is rewritten

$$
\dot{\gamma} j \Psi+\dot{\Psi}=C \chi+Q[\chi] .
$$


We now apply the spectral projections $P_{0}, P_{1}$ and $P_{c}$ to (2.2.5) and get a system of coupled equations for $\omega(t), \gamma(t), z(t)$ and $f(x, t)$ in the form

Proposition 2.2. - The functions $\omega(t), \gamma(t), z(t)$ and $f(x, t)$ satisfy the system

$$
\begin{aligned}
& \dot{\omega}=\frac{\left\langle P_{0} Q, \Psi\right\rangle}{\left\langle\left(\phi_{\omega}-P_{0 \omega} \chi\right), \Psi\right\rangle}, \\
& \dot{\gamma}=\frac{\left\langle j P_{0}\left(\phi_{\omega}-P_{0 \omega} \chi\right), P_{0} Q\right\rangle}{\left\langle\left(\phi_{\omega}-P_{0 \omega} \chi\right), \Psi\right\rangle}, \\
& \langle u, j u\rangle(\dot{z}-i \mu z)=\langle Q, j u\rangle-\left\langle w_{\omega}-P_{1 \omega} f, j u\right\rangle \dot{\omega}-\langle\chi, u\rangle \dot{\gamma}, \\
& \dot{f}=C f+P_{c} Q[\chi]+\dot{\omega} P_{c \omega} \chi-\dot{\gamma} P_{c}(j \chi) .
\end{aligned}
$$

Proof. - Taking the scalar product of (2.2.5) with $P_{0}^{*} \Psi$ leads to

$$
\dot{\gamma}\left\langle j \Psi, P_{0}^{*} \Psi\right\rangle+\left\langle\dot{\Psi}, P_{0}^{*} \Psi\right\rangle=\left\langle P_{0} Q, \Psi\right\rangle .
$$

Since

$$
\left\langle j \Psi, P_{0}^{*} \Psi\right\rangle=\left\langle j \Psi, P_{0}^{* 2} \Psi\right\rangle=\left\langle P_{0} j \Psi, P_{0}^{*} \Psi\right\rangle=\left\langle j P_{0}^{*} \Psi, P_{0}^{*} \Psi\right\rangle=0,
$$

we have,

$$
\left\langle P_{0} \dot{\Psi}, \Psi\right\rangle=\left\langle P_{0} Q, \Psi\right\rangle .
$$

Notice also that $\Psi=\phi+\chi$ and that $P_{0} \chi=0$. Therefore,

$$
P_{0} \dot{\chi}+\dot{\omega} P_{0 \omega} \chi=0
$$

and

$$
P_{0} \dot{\Psi}=\left(\phi_{\omega}-P_{0 \omega} \chi\right) \dot{\omega} .
$$

This immediately implies (2.2.6).

Taking the scalar product of (2.2.5) with $j P_{0} \dot{\Psi}$, we get

$$
\dot{\gamma}\left\langle\Psi, P_{0} \dot{\Psi}\right\rangle=\left\langle Q, j P_{0} \dot{\Psi}\right\rangle
$$

Substituting (2.2.14) in the above equation leads to (2.2.7).

Applying $P_{1}$ to (2.2.5) gives

$$
P_{1} \dot{\Psi}+P_{1}(j \Psi) \dot{\gamma}=C w+P_{1} Q[\chi]
$$

with $P_{1}(j \Psi)=P_{1}(j \chi)$ and $P_{1} \dot{\Psi}=P_{1} \dot{w}+P_{1} \dot{f}$. Since $P_{1} w=w$, we have

$$
\dot{w}=P_{1 \omega} \dot{\omega} w+P_{1} \dot{w} .
$$

Using that $P_{1}^{2}=P_{1}$, we have $P_{1 \omega} w=0$, thus $\dot{w}=P_{1} \dot{w}$. From $P_{1} f=0$, we get that

$$
P_{1} \dot{f}=-P_{1 \omega} f \dot{\omega}=-P_{1} P_{1 \omega} f \dot{\omega} .
$$


Finally, (2.2.16) is rewritten in the form

$$
P_{1} \dot{w}-P_{1} P_{1 \omega} f \dot{\omega}+\dot{\gamma} P_{1} j \chi=C w+P_{1} Q[\chi] .
$$

Using that $w=z u+\bar{z} u^{*}$, and that $u$ and $u^{*}$ depend on $t$ through $\omega$, we can write $\dot{w}=w_{t}+\dot{\omega} w_{\omega}$. Eq. (2.2.16) becomes

$$
w_{t}+\dot{\omega} P_{1}\left(w_{\omega}-P_{1 \omega} f\right)+\dot{\gamma} P_{1}(j \chi)=C w+P_{1} Q[\chi]
$$

Using that $w_{t}=\dot{z} u+\dot{\bar{z}} u^{*}$ and $C w=i \mu\left(z u-\bar{z} u^{*}\right)$ in (2.2.20), we get, after taking the scalar product of this equation with $j u$,

$$
\langle u, j u\rangle(\dot{z}-i \mu z)=\langle Q, j u\rangle-\left\langle w_{\omega}-P_{1 \omega} f, j u\right\rangle \dot{\omega}-\langle\chi, u\rangle \dot{\gamma}
$$

Finally, when the projection $P_{c}$ is applied to (2.2.5), one gets

$$
\dot{\gamma} P_{c}(j \Psi)+P_{c} \dot{\Psi}=C f+P_{c} Q
$$

Notice that $P_{c} j \phi=0$ and $P_{c} \dot{\phi}=P_{c} \phi_{\omega} \dot{\omega}=0$. Therefore

$$
\dot{\gamma} P_{c}(j \chi)+P_{c} \dot{\chi}=C f+P_{c} Q
$$

Furthermore, $P_{c} \dot{w}=-P_{c \omega} w \dot{\omega}, P_{c} \dot{f}=\dot{f}-P_{c \omega} f \dot{\omega}$, and $P_{c} \dot{\chi}=\dot{f}-P_{c \omega} \chi \dot{\omega}$. Substituting $P_{c} \dot{\chi}$ in (2.2.22) by the above expression, we get (2.2.9).

\section{Leading terms and remainders}

\subsection{Preliminaries}

This section is devoted to some preliminary useful estimates. We start with a bound for the denominator $\left\langle P_{0}\left(\phi_{\omega}-P_{0 \omega} \chi\right), \Psi\right\rangle$ that appears in the equations of motion (2.2.6)(2.2.9). We have

$$
\left\langle P_{0}\left(\phi_{\omega}-P_{0 \omega} \chi\right), \Psi\right\rangle=\left\langle\phi_{\omega}, \phi\right\rangle\left(1-\frac{\left\langle P_{0 \omega}(w+f), \phi\right\rangle}{\left\langle\phi_{\omega}, \phi\right\rangle}\right)
$$

with

$$
\frac{\left\langle P_{0 \omega} \chi, \phi\right\rangle}{\left\langle\phi_{\omega}, \phi\right\rangle}=\mathcal{R}(\omega)\left(|z|+\int \mathrm{e}^{-\sqrt{\omega}|x|}|f(x)| d x\right)
$$

where $\mathcal{R}(\omega)$ is a general notation for functions which remain bounded if $\omega$ is close to $\omega_{0}$. It could be unbounded and even infinite if $\omega$ is outside some vicinity of $\omega_{0}$. The formula $f=\mathcal{R} g$ implies that $|f| \leqslant \mathcal{R} g$. We have

$$
\frac{\left\langle P_{0 \omega} \chi, \phi\right\rangle}{\left\langle\phi_{\omega}, \phi\right\rangle}=\mathcal{R}(\omega)\left(|z|+\|f\|_{\rho}\right)
$$


where $\rho(x)=\left(1+x^{2}\right)^{-\alpha}, \alpha$ arbitrary. For $\alpha>1 / 4,\|f\|_{\rho} \leqslant C\|f\|_{\infty}$, and

$$
\frac{\left\langle P_{0 \omega} \chi, \phi\right\rangle}{\left\langle\phi_{\omega}, \phi\right\rangle}=\mathcal{R}(\omega)\left(|z|+\|f\|_{\infty}\right) .
$$

In the following, we will always assume $\alpha>1 / 4$ in the definition of $\rho$. We also need to expand the nonlinear term $F\left(|\psi|^{2}\right) \psi$ near the solitary wave. The Taylor expansion of $F\left(|\psi|^{2} \psi\right.$ near $\phi$ is

$$
F\left(|\psi|^{2}\right) \psi=F\left(|\phi|^{2}\right) \phi+F\left(|\phi|^{2}\right) \chi+2 F^{\prime}\left(|\phi|^{2}\right)(\chi, \phi) \phi+E[\chi],
$$

where $E[\chi]$ contains all the higher order terms which are at least quadratic in $\chi$, as $\chi \rightarrow 0$. Recalling that $r$ is the order of the zero of $F(s)$ at $s=0$, we expand $E[\chi]$ in the form

$$
E[\chi]=E_{2}+\cdots+E_{2 r}+E_{R}
$$

where $E_{j}$ is of order $j$ in $\chi$ and $E_{R}$ the remainder. For $j \leqslant 2 r$, all the terms $E_{j}$ contain powers of $\varphi$ (which decreases exponentially fast), thus

$$
E_{j}=\mathcal{R}(x, \omega)|\chi|^{j},
$$

where $\mathcal{R}(x, \omega)$ denotes functions that satisfy the estimate

$$
|\mathcal{R}(x, \omega)| \leqslant \mathcal{R}(\omega) \mathrm{e}^{-\sqrt{\omega}|x|} .
$$

However for $E_{R}$, we have only

$$
\left|E_{R}\right|=\mathcal{R}(\omega,|\chi(x)|)|\chi|^{2 r+1}=\mathcal{R}\left(\omega,|z|+\|f\|_{\infty}\right)|\chi|^{2 r+1} .
$$

The notation $\mathcal{R}$ when $\mathcal{R}$ depends on several variables has the same meaning as when it depends on one variable only.

More explicitly, the quadratic terms have the form

$$
E_{2}[\chi, \chi]=F^{\prime}\left(|\phi|^{2}\right)|\chi|^{2} \phi+2 F^{\prime \prime}\left(|\phi|^{2}\right)(\phi, \chi)^{2} \phi+2 F^{\prime}\left(|\phi|^{2}\right)(\phi, \chi) \chi
$$

and the cubic terms

$$
\begin{aligned}
E_{3}[\chi, \chi, \chi]= & \frac{4}{3} F^{\prime \prime \prime}\left(|\phi|^{2}\right)(\phi, \chi)^{3} \phi+2 F^{\prime \prime}\left(|\phi|^{2}\right)(\phi, \chi)^{2} \chi+F^{\prime}\left(|\phi|^{2}\right)|\chi|^{2} \chi \\
& +2 F^{\prime \prime}\left(|\phi|^{2}\right)(\phi, \chi)|\chi|^{2} \phi .
\end{aligned}
$$

It is also useful to define $E_{2}\left[\chi_{1}, \chi_{2}\right]$ as a symmetric quadratic form

$$
\begin{aligned}
E_{2}\left[\chi_{1}, \chi_{2}\right]= & \frac{1}{2} F^{\prime}\left(|\phi|^{2}\right)\left(\left(\chi_{1}, \chi_{2}\right)+\left(\chi_{2}, \chi_{1}\right)\right) \phi+2 F^{\prime \prime}\left(|\phi|^{2}\right)\left(\phi, \chi_{1}\right)\left(\phi, \chi_{2}\right) \phi \\
& +F^{\prime}\left(|\phi|^{2}\right)\left(\left(\phi, \chi_{2}\right) \chi_{1}+\left(\phi, \chi_{1}\right) \chi_{2}\right) .
\end{aligned}
$$

Notice that $E_{2}$ satisfies the following property

$$
\left\langle E_{2}[X, Y], Z\right\rangle=\left\langle X, E_{2}\left[Y^{*}, Z\right]\right\rangle
$$


where $X, Y, Z$ are complex valued vector functions and $X^{*}=\left(\bar{X}_{1}, \bar{X}_{2}\right)$.

\subsection{Leading terms and remainders for $\dot{\gamma}, \dot{\omega}$ and $\dot{z}$}

Using the calculations of the previous section, we can now rewrite Eqs. (2.2.6)-(2.2.8) describing the time evolution of $\gamma, \omega$ and $z$ and separate leading terms and remainders. We get

$$
\begin{aligned}
\dot{\gamma}= & -\frac{\left\langle E_{2}[w, w]+2 E_{2}[w, f], \phi_{\omega}\right\rangle}{\left\langle\phi, \phi_{\omega}\right\rangle} \\
& -\left\langle\phi, \phi_{\omega}\right\rangle^{-2}\left[\left(\left\langle E_{3}[w, w, w], \phi_{\omega}\right\rangle-\left\langle E_{2}[w, w], P_{0 \omega} w\right\rangle\right)\left\langle\phi, \phi_{\omega}\right\rangle\right. \\
& \left.+\left\langle E_{2}[w, w], \phi_{\omega}\right\rangle\left(\left\langle P_{0 \omega} w, \phi\right\rangle-\left\langle w, \phi_{\omega}\right\rangle\right)\right]+\Gamma_{R},
\end{aligned}
$$

where

$$
\Gamma_{R}=\mathcal{R}\left(\omega,|z|+\|f\|_{\infty}\right)\left(|z|^{2}+\|f\|_{\rho}\right)^{2} .
$$

In the above equation, $\mathcal{R}\left(\omega,|z|+\|f\|_{\infty}\right)$ is a quantity that remains bounded if $\omega$ is in the vicinity of $\omega_{0}$ and if $|z|+\|f\|_{\infty}$ is bounded. Similarly, Eq. (2.2.6) for $\omega$ is rewritten

$$
\begin{aligned}
\dot{\omega}= & \frac{\left\langle E_{2}[w, w]+2 E_{2}[w, f], j \phi\right\rangle}{\left\langle\phi, \phi_{\omega}\right\rangle} \\
& +\left\langle\phi, \phi_{\omega}\right\rangle^{-2}\left[\left(\left\langle E_{3}[w, w, w], j \phi\right\rangle+\left\langle E_{2}[w, w], P_{0} j \chi\right\rangle\right)\left\langle\phi, \phi_{\omega}\right\rangle\right. \\
& \left.+\left\langle E_{2}[w, w], j \phi\right\rangle\left(\left\langle P_{0 \omega} w, \phi\right\rangle-\left\langle\phi_{\omega}, w\right\rangle\right)\right]+\Omega_{R},
\end{aligned}
$$

where

$$
\Omega_{R}=\mathcal{R}\left(\omega,|z|+\|f\|_{\infty}\right)\left(|z|^{2}+\|f\|_{\rho}\right)^{2} .
$$

It is useful to notice that

$$
\left\langle E_{2}[w, w], j \phi\right\rangle=z^{2}\left\langle E_{2}[u, u], j \phi\right\rangle+\bar{z}^{2}\left\langle E_{2}\left[u^{*}, u^{*}\right], j \phi\right\rangle+2 z \bar{z}\left\langle E_{2}\left[u, u^{*}\right], j \phi\right\rangle .
$$

Using the definition of $E_{2}$, and that $u=\left(u_{1}, u_{2}\right)$ with $u_{1}$ real and $u_{2}$ pure imaginary, we have that

$$
\left\langle E_{2}\left[u, u^{*}\right], j \phi\right\rangle=0 .
$$

Thus,

$$
\left\langle E_{2}[w, w], j \phi\right\rangle=\left(z^{2}-\bar{z}^{2}\right)\left\langle E_{2}[u, u], j \phi\right\rangle
$$

and

$$
\left\langle E_{2}[u, u], j \phi\right\rangle=\frac{1}{\left\langle\phi, \phi_{\omega}\right\rangle} 2 \int F^{\prime}\left(|\phi|^{2}\right)(u, \phi)(u, j \phi) d x
$$

is purely imaginary.

Finally, we rewrite (2.2.8) in the form:

$$
\begin{aligned}
\dot{z}-i \mu z= & -\frac{\left\langle E_{2}[w, w]+2 E_{2}[w, f]+E_{3}[w, w, w], u\right\rangle}{\langle u, j u\rangle} \\
& -\frac{\left\langle w_{\omega}, j u\right\rangle\left\langle E_{2}[w, w], j \phi\right\rangle-\langle w, u\rangle\left\langle E_{2}[w, w], \phi_{\omega}\right\rangle}{\left\langle\phi, \phi_{\omega}\right\rangle\langle u, j u\rangle}+Z_{R},
\end{aligned}
$$


where

$$
Z_{R}=\mathcal{R}\left(\omega,|z|+\|f\|_{\infty}\right)\left(|z|^{2}+\|f\|_{\rho}\right)^{2} .
$$

Proposition 3.1. $-\langle u, j u\rangle=i \delta$, with $\delta>0$.

Proof. - Indeed,

$$
\langle u, j u\rangle=\int_{\mathbf{R}} u_{1}\left(u_{2}-u_{2}^{*}\right) d x=2 \int_{\mathbf{R}} u_{1} u_{2} d x .
$$

Using (2.1.5),

$$
\langle u, j u\rangle=\frac{2}{i \mu} \int u_{2} \mathcal{D}_{2} u_{2} d x=\frac{2}{i \mu} \int u_{2}\left(-\frac{d^{2}}{d x^{2}}+\omega+F\left(|\phi|^{2}\right)\right) u_{2} d x .
$$

Since $u_{2}$ is purely imaginary and $\phi$ is the only eigenfunction of the operator $-\frac{d^{2}}{d x^{2}}+\omega+$ $F\left(|\phi|^{2}\right)$ corresponding to the minimal spectral point 0 , the integral is strictly negative and the result follows.

Remark. - It is shown in [2] that under Assumption (SP), $\left\langle\phi, \phi_{\omega}\right\rangle>0$ for $\omega=\omega_{0}$ and consequently for $\omega$ close to $\omega_{0}$.

\subsection{Leading terms and remainders for $\dot{f}$}

We now turn to Eq. (2.2.9) for $f$ that we rewrite in the form

$$
\dot{f}=C f-P_{c} j E_{2}[w, w]+\dot{\gamma} i\left(P_{+}-P_{-}\right) f+F_{R}
$$

where the remainder $F_{R}$ is

$$
F_{R}=-P_{c} j\left(E[\chi]-E_{2}[w, w]\right)-\dot{\omega} P_{d \omega} \chi+\dot{\gamma} P_{c} j^{-1} w+\dot{\gamma}\left(P_{c} j^{-1}-i\left(P_{+}-P_{-}\right)\right) f .
$$

As we have seen before,

$$
P_{c} Q[\chi]=-P_{c} j E[\chi]
$$

and

$$
\begin{aligned}
E[\chi]= & E_{2}[w, w]+\mathcal{R}\left(x ; \omega,|z|+\|f\|_{\infty}\right)\left(|z|^{3}+|z||f|+|f|^{2}\right) \\
& +\mathcal{R}\left(\omega,|z|+\|f\|_{\infty}\right)|\chi|^{2 r+1} .
\end{aligned}
$$

Denoting $E_{R}=\mathcal{R}\left(\omega,|z|+\|f\|_{\infty}\right)|\chi|^{2 r+1}$ we have

$$
P_{c} j E_{R}=j E_{R}-P_{d} j E_{R}
$$

with

$$
P_{d} j E_{R}=\mathcal{R}\left(x ; \omega,|z|+\|f\|_{\infty}\right)\left(|z|^{2 r+1}+\|f\|_{\infty}^{2 r-1}\|f\|_{\rho}^{2}\right) .
$$

We rewrite the remainder $F_{R}$ as 


$$
\begin{aligned}
F_{R}= & -P_{c} j\left(E[\chi]-E_{2}[w, w]\right)-\dot{\omega} P_{d \omega} \chi+\dot{\gamma} P_{c} j^{-1} w \\
& +\dot{\gamma}\left(P_{c} j^{-1}-i\left(P_{+}-P_{-}\right)\right) f .
\end{aligned}
$$

To estimate $F_{R}$, we separate it in several parts as follows:

$$
F_{R}=F_{1}+F_{2}+F_{3}+F_{4}+F_{5}
$$

with

$$
\begin{aligned}
& F_{1}=-P_{c} j\left(E_{2}[\chi]+\cdots+E_{2 r}[\chi]-E_{2}[w, w]\right), \\
& F_{2}=-P_{c} j E_{R}, \\
& F_{3}=\dot{\omega} P_{c \omega} \chi=-\dot{\omega} P_{d \omega} \chi, \\
& F_{4}=\dot{\gamma} P_{c} j w=\dot{\gamma} j w-\dot{\gamma} P_{d} j w, \\
& F_{5}=\dot{\gamma}\left[P_{c} j^{-1}-i\left(P_{+}-P_{-}\right)\right] f .
\end{aligned}
$$

It is convenient to separate $F_{2}$ in two parts,

$$
F_{2}=F_{2}^{\prime}+F_{2}^{\prime \prime}
$$

with

$$
\begin{aligned}
& F_{2}^{\prime}=-j E_{R}, \\
& F_{2}^{\prime \prime}=P_{d} j E_{R} .
\end{aligned}
$$

PROPOSITION 3.2. - We have the following estimates:

$$
\begin{aligned}
\left\|\left(1+x^{2}\right) F_{1}\right\|_{2} & =\mathcal{R}\left(\omega,|z|+\|f\|_{\infty}\right)\left(|z|^{3}+|z|\|f\|_{\rho}+\|f\|_{\infty}\|f\|_{\rho}\right), \\
\left\|\left(1+x^{2}\right) F_{2}^{\prime \prime}\right\|_{2} & =\mathcal{R}\left(\omega,|z|+\|f\|_{\infty}\right)\left(|z|^{2 r+1}+\|f\|_{\infty}\left\|^{2 r}\right\| f \|_{\rho}\right), \\
\left\|\left(1+x^{2}\right) F_{3}\right\|_{2} & =|\mathcal{R}(\omega)||\dot{\omega}|\left(|z|+\|f\|_{\infty}\right), \\
\left\|\left(1+x^{2}\right) F_{4}\right\|_{2} & =\mathcal{R}(\omega)|\dot{\gamma} \| z|, \\
\left\|\left(1+x^{2}\right) F_{5}\right\|_{2} & =\mathcal{R}(\omega)|\dot{\gamma}|\|f\|_{\rho} .
\end{aligned}
$$

Proof. - Estimates for $F_{1}, F_{2}^{\prime \prime}, F_{3}, F_{4}$, are straightforward. Inequality (3.3.21) for $F_{5}$ is a consequence of Proposition 2.1.

PROPOSITION 3.3. - The term $F_{2}^{\prime}$ is estimated as follows:

$$
\begin{aligned}
& \left\|F_{2}^{\prime}\right\|_{2}=\mathcal{R}\left(\omega,|z|+\|f\|_{\infty}\right)\left(|z|^{2 r}+\|f\|_{L^{\infty}}^{2 r}\right), \\
& \left\|\left(1+x^{2}\right) F_{2}^{\prime}\right\|_{1}=\mathcal{R}\left(\omega,|z|+\|f\|_{\infty}\right)(1+t)\left(|z|+\|f\|_{\infty}\right)^{2 r-1} .
\end{aligned}
$$

Proof. - First consider $\left\|F_{2}^{\prime}\right\|_{2}$. We have

$$
\begin{aligned}
\left\|F_{2}^{\prime}\right\|_{2}^{2} & =\mathcal{R}\left(\omega,|z|+\|f\|_{\infty}\right) \int|\chi|^{2(2 r+1)} d x \\
& \leqslant \mathcal{R}\left(\omega,|z|+\|f\|_{\infty}\right)\left(|z|+\|f\|_{\infty}\right)^{4 r}\|\chi\|_{2}^{2} .
\end{aligned}
$$

Using the conservation of the $L_{2}$-norm of $\psi$, we have

$$
\|\chi\|_{L^{2}} \leqslant C+\mathcal{R}(\omega) .
$$


After substitution in the estimate for $\left\|F_{2}^{\prime}\right\|_{2}$, we get (3.3.22). To obtain (3.3.23), we use the classical estimate on the weighted $L^{2}$-norm of the solution [6]

$$
\left(\int\left(1+x^{2}\right)|\psi|^{2} d x\right)^{1 / 2} \leqslant C\left(\left\|\psi_{0}\right\|_{H^{1}}\right)(1+t)\left\|(1+|x|) \psi_{0}\right\|_{2}
$$

It follows that

$$
\left\|\left(1+x^{2}\right) F_{2}^{\prime}\right\|_{1}=\mathcal{R}\left(\omega,|z|+\|f\|_{\infty}\right)(1+t)\left(|z|+\|f\|_{\infty}\right)^{2 r-1} .
$$

These estimates are sufficient to bound $\|f\|_{\infty}$, but not $\|f\|_{\rho}, \omega, \gamma$ and $z$. For the latter, we need to consider the leading terms more carefully. This is done in the next section.

We conclude by summarizing the results of this section. Let us rewrite $F_{R}=F_{\mathrm{I}}+F_{\mathrm{II}}$, with

$$
F_{\mathrm{I}}=F_{1}+F_{2}^{\prime \prime}+F_{3}+F_{4}+F_{5}, \quad \text { and } \quad F_{\mathrm{II}}=F_{2}^{\prime} .
$$

PROPOSITION 3.4. - The evolution equation for $f$ is

$$
\dot{f}=C(\omega) f-P_{c} j E_{2}[w, w]+i \dot{\gamma}\left(P_{+}-P_{-}\right) f+F_{R}
$$

with a remainder $F_{R}=F_{\mathrm{I}}+F_{\mathrm{II}}$ satisfying

$$
\begin{aligned}
& \left\|\left(1+x^{2}\right) F_{\mathrm{I}}\right\|_{2}=\mathcal{R}\left(\omega,|z|+\|f\|_{\infty}\right)\left(|z|^{3}+\left(|z|+\|f\|_{\infty}\right)\|f\|_{\rho}\right), \\
& \left\|F_{\mathrm{II}}\right\|_{2}+\left\|\left(1+x^{2}\right) F_{\mathrm{II}}\right\|_{1}=\mathcal{R}\left(\omega,|z|+\|f\|_{\infty}\right)(1+t)\left(|z|+\|f\|_{\infty}\right)^{2 r-1} .
\end{aligned}
$$

\section{Transformation of the evolution equations}

Our goal is to transform the evolution equations for $\gamma, \omega, z$ and $f$ to a more simple, canonical form. We will use the idea of normal coordinates, trying to keep unchanged the estimates for the remainders.

\subsection{Equation for $\omega$}

Eq. (3.2.3) can be represented in the form

$$
\begin{aligned}
\dot{\omega}= & \Omega_{20}(\omega) z^{2}+\Omega_{11}(\omega) z \bar{z}+\Omega_{02}(\omega) \bar{z}^{2}+\Omega_{30}(\omega) z^{3}+\Omega_{21}(\omega) z^{2} \bar{z} \\
& +\Omega_{12}(\omega) z \bar{z}^{2}+\Omega_{03} \bar{z}^{3}+z\left\langle f, \Omega_{10}^{\prime}\right\rangle+\bar{z}\left\langle f, \Omega_{01}^{\prime}\right\rangle+\Omega_{R} .
\end{aligned}
$$

Notice that $\Omega_{i j}=\bar{\Omega}_{j i}$. Furthermore,

$$
\Omega_{20}=\bar{\Omega}_{02}=\frac{\left\langle E_{2}[u, u], j \phi\right\rangle}{\left\langle\phi, \phi_{\omega}\right\rangle}=\frac{2}{\left\langle\phi, \phi_{\omega}\right\rangle} \int d x F^{\prime}\left(|\phi|^{2}\right)(\phi, u)(u, j \phi)
$$


is purely imaginary and $\Omega_{11}=-\frac{\left\langle E_{2}\left[u, u^{*}\right], j \phi\right\rangle}{\left\langle\phi, \phi_{\omega}\right\rangle}=0$ (see (3.2.6)). Using the property (3.1.13), we find that the coefficients $\Omega_{10}^{\prime}$ and $\Omega_{01}^{\prime}$ are given by

$$
\Omega_{10}^{\prime}=\bar{\Omega}_{01}^{\prime}=2 \frac{E_{2}\left[u^{*}, j \phi\right]}{\left\langle\phi, \phi_{\omega}\right\rangle} .
$$

Let us now consider the equation for $z$. It has the form

$$
\begin{aligned}
\dot{z}= & i\left(\mu z+Z_{20} z^{2}+Z_{11} z \bar{z}+Z_{02} \bar{z}^{2}+Z_{30} z^{3}+Z_{21} z^{2} \bar{z}+Z_{12} z \bar{z}^{2}+Z_{03} \bar{z}^{3}\right) \\
& +z\left\langle f, Z_{10}^{\prime}\right\rangle+\bar{z}\left\langle f, Z_{01}^{\prime}\right\rangle+Z_{R} .
\end{aligned}
$$

We have

$$
\begin{array}{ll}
Z_{20}=\frac{\left\langle E_{2}[u, u], u\right\rangle}{\langle u, j u\rangle}, & Z_{11}=\frac{\left\langle E_{2}\left[u, u^{*}\right], u\right\rangle}{\langle u, j u\rangle}, \quad Z_{02}=\frac{\left\langle E_{2}\left[u^{*}, u^{*}\right], u^{*}\right\rangle}{\langle u, j u\rangle}, \\
Z_{10}^{\prime}=-2 \frac{E_{2}\left[u^{*}, u\right]}{\langle u, j u\rangle}, & Z_{01}^{\prime}=-2 \frac{E_{2}[u, u]}{\langle u, j u\rangle} .
\end{array}
$$

Proposition 4.1. - There exist coefficients $b_{i j}(\omega), 0 \leqslant i, j \leqslant 3$, and vector-functions $b_{i j}^{\prime}(x, \omega)$, such that the new function $\omega_{1}$ defined as

$$
\begin{aligned}
\omega_{1}= & \omega+b_{20}(\omega) z^{2}+b_{02}(\omega) \bar{z}^{2}+b_{30}(\omega) z^{3}+b_{21}(\omega) z^{2} \bar{z}+b_{12} z \bar{z}^{2} \\
& +b_{03} \bar{z}^{3}+z\left\langle f, b_{10}^{\prime}\right\rangle+\bar{z}\left\langle f, b_{01}^{\prime}\right\rangle
\end{aligned}
$$

obeys the differential equation

$$
\dot{\omega}_{1}=\widehat{\Omega_{R}}
$$

and $\widehat{\Omega_{R}}$ satisfies the same estimate (3.2.4) as $\Omega_{R}$.

Proof. - The calculation follows the classical method of normal coordinates. Substituting $\dot{\omega}, \dot{z}, \dot{\bar{z}}$, and $\dot{f}$ from (4.1.1), (4.1.4), (3.3.29) into the equation for $\dot{\omega}_{1}$, we get

$$
\begin{aligned}
\dot{\omega}_{1}= & \left(1+b_{20 \omega} z^{2}+b_{02 \omega} \bar{z}^{2}+b_{30 \omega} z^{3}+b_{21 \omega} z^{2} \bar{z}+b_{12 \omega} z \bar{z}^{2}+b_{03} \bar{z}^{3}\right. \\
& \left.+z\left\langle f, b_{10 \omega}^{\prime}\right\rangle+\bar{z}\left\langle f, b_{01 \omega}^{\prime}\right\rangle\right) \dot{\omega}+\left(2 b_{20} z+3 b_{30} z^{2}+2 b_{21} z \bar{z}+b_{12} \bar{z}^{2}+\left\langle f, b_{10}^{\prime}\right\rangle\right) \dot{z} \\
& +\left(2 b_{02} \bar{z}+b_{21} z^{2}+2 b_{12} z \bar{z}+3 b_{03} \bar{z}^{2}+\left\langle f, b_{01}^{\prime}\right\rangle\right) \dot{\bar{z}}+z\left\langle\dot{f}, b_{10}^{\prime}\right\rangle+\bar{z}\left\langle\dot{f}, b_{01}^{\prime}\right\rangle \\
= & \Omega_{20} z^{2}+\Omega_{02} \bar{z}^{2}+\Omega_{30} z^{3}+\Omega_{21} z^{2} \bar{z}+\Omega_{12} z \bar{z}^{2}+\Omega_{03} \bar{z}^{3}+z\left\langle f, \Omega_{10}^{\prime}\right\rangle \\
& +\bar{z}\left\langle f, \Omega_{01}^{\prime}\right\rangle+\Omega_{R}+2 b_{20} z\left(i \mu z+i Z_{20} z^{2}+i Z_{11} z \bar{z}+i Z_{02} \bar{z}^{2}\right) \\
& +\left(3 b_{30} z^{2}+2 b_{21} z \bar{z}+b_{12} \bar{z}^{2}+\left\langle f, b_{10}^{\prime}\right\rangle\right)(i \mu z) \\
& +2 b_{02} \bar{z}\left(-i \mu \bar{z}-i Z_{20} \bar{z}^{2}-i Z_{11} \bar{z} z-i Z_{02} z^{2}\right) \\
& +\left(b_{21} z^{2}+2 b_{12} z \bar{z}+3 b_{03} \bar{z}^{2}+\left\langle f, b_{01}^{\prime}\right\rangle\right)(-i \mu \bar{z}) \\
& +z\left\langle C f+i \dot{\gamma}\left(P_{+}-P_{-}\right) f+F_{20} z^{2}+F_{11} z \bar{z}+F_{02} \bar{z}^{2}+F_{R}, b_{10}^{\prime}\right\rangle \\
& +\bar{z}\left\langle C f+i \dot{\gamma}\left(P_{+}-P_{-}\right) f+F_{20} z^{2}+F_{11} z \bar{z}+F_{02} \bar{z}^{2}+F_{R}, b_{01}^{\prime}\right\rangle \\
& +\mathcal{R}\left(\omega,|z|+\|f\|_{\infty}\right)\left(|z|^{4}+|z|^{2}\|f\|_{\rho}\right) \\
= & \widehat{\Omega_{R}} .
\end{aligned}
$$


Comparison of the coefficients of $z^{2}, \bar{z}^{2}, z^{3}, z^{2} \bar{z}, z \bar{z}^{2}, \bar{z}^{3}, z f$ and $\bar{z} f$, leads to a system of equations for the coefficients $b_{i j}$ and $b_{i j}^{\prime}$. In particular,

$$
\begin{aligned}
& b_{20}=\frac{i}{2 \mu} \Omega_{20}, \quad b_{02}=-\frac{i}{2 \mu} \Omega_{02}, \\
& \Omega_{30}+2 i Z_{20} b_{20}+3 i \mu b_{30}+\left\langle F_{02}, b_{10}^{\prime}\right\rangle=0, \\
& \Omega_{21}+2 i Z_{11} b_{20}+i \mu b_{21}-2 i Z_{02} b_{02}+\left\langle F_{11}, b_{10}^{\prime}\right\rangle+\left\langle F_{20}, b_{10}^{\prime}\right\rangle=0, \\
& \Omega_{10}^{\prime}-i \mu b_{10}^{\prime}+C^{*} b_{10}^{\prime}=0
\end{aligned}
$$

and thus,

$$
b_{10}^{\prime}=-\left(C^{*}-i \mu\right)^{-1} \Omega_{10}^{\prime} .
$$

The coefficient $\Omega_{10}^{\prime} \in X_{c}$ and we can require that $b_{10}^{\prime}$ belongs to $X_{c}$.

Equations for $b_{30}$ and $b_{21}$ can be solved once $b_{02}$ and $b_{10}^{\prime}$ have been calculated. It follows that

$$
\begin{aligned}
\widehat{\Omega}_{R} & =\Omega_{R}+\mathcal{R}\left(\omega,|z|+\|f\|_{\infty}\right)\left(|z|^{4}+|z|^{2}\|f\|_{\rho}\right) \\
& =\mathcal{R}\left(\omega,|z|+\|f\|_{\infty}\right)\left(|z|^{2}+\|f\|_{\rho}\right)^{2} .
\end{aligned}
$$

Remark. - If we apply the same method to the equation for $z$ (see (4.1.4)), a change of variables similar to (4.1.9)-(4.1.12) leads to a system of equations for the coefficients $c_{i j}$ of $z$. The analogous equation of (4.1.13) for the coefficients $c_{01}^{\prime}$ has now the form

$$
\left(C^{*}+2 i \mu\right) c_{01}^{\prime}=-i Z_{10}^{\prime}
$$

Note that $-2 i \mu$ is a point in the continuous spectrum of $C^{*}$, thus the function $c_{01}^{\prime}$ does not vanish at infinity. This implies that in the expression for the new variable $z_{1}$, the term $\bar{z}\left\langle f, c_{01}^{\prime}\right\rangle$ can have a complicated structure. The function $f$ itself does not decrease well enough at infinity. To proceed with the equation for $z$, we have to analyze carefully the behavior of $f$.

\subsection{Transformation of the equation for $f$}

\subsubsection{Decomposition of $f$}

In Section 3.3, we transformed the evolution equation (2.2.9) for $f$ into (3.3.29) with estimates on the remainder. The difficulty however is that $\omega$ is not a constant (it is a function of $t$ ) and $C(\omega)$ and $P_{c} j$ are not in simple relation. For this reason, let us come back to (2.2.9) rewritten in the form

$$
\dot{f}=C(\omega) f+\dot{\gamma} P_{c} j^{-1} f-P_{c} j E_{2}[w, w]+\widehat{F_{R}},
$$

where $\widehat{F_{R}}=\widehat{F_{\mathrm{I}}}+F_{\mathrm{II}}$ and $\widehat{F_{\mathrm{I}}}=F_{1}+F_{2}^{\prime \prime}+F_{3}+F_{4}$. Let us fix a time interval $[0, T]$ and decompose $f$ into

$$
f=g+h
$$

with $g \in X_{T}^{d}$ and $h \in X_{T}^{c}$, where $X_{T}^{d}=P_{T}^{d} X$ is the spectral space associated to the discrete spectrum at time $T$ and $X_{T}^{c}=P_{T} X$ the spectral space associated to the 
continuous spectrum at time $T, P_{T}=P_{c}(\omega(T))$ and $P_{T}^{d}=I-P_{T}$. In the following, we denote $\omega_{T}=\omega(T)$ and $C_{T}=C\left(\omega_{T}\right)$. Notice that $g_{\mid t=T}=0$.

One has

$$
P_{d}(\omega) f=0
$$

which implies that

$$
g+\left(P_{d}-P_{T}^{d}\right) g+\left(P_{d}-P_{T}^{d}\right) h=0 .
$$

This will be used to estimate $g$ in terms of $h$, since $P_{d}-P_{T}^{d}$ is a 'small' operator in any reasonable norm.

Introducing the notation $\mathcal{R}_{1}(\omega)=\mathcal{R}\left(\left\|\omega-\omega_{0}\right\|_{C[0, T]}\right)$, we have

Proposition 4.2. - The function $g$ is estimated in terms of $h$ as follows:

$$
\begin{aligned}
& \|g\|_{\rho}=\mathcal{R}_{1}(\omega)\left|\omega_{T}-\omega\right|\|h\|_{\rho}, \\
& \|g\|_{\infty}=\mathcal{R}_{1}(\omega)\left|\omega_{T}-\omega\right|\|h\|_{\rho} .
\end{aligned}
$$

Notice that

$$
\|h\|_{\rho}=\left\|P_{T} f\right\|_{\rho}=\left\|\left(1-P_{T}^{d}\right) f\right\|_{\rho}=\mathcal{R}_{1}(\omega)\|f\|_{\rho} .
$$

Applying the projection $P_{T}$ to (4.2.1), we get

$$
\dot{h}=C_{T} h+\sigma(t) P_{T} j^{-1} h-P_{T} j E_{2}[w, w]+H_{R}^{\prime}
$$

with $\sigma(t)=\omega-\omega_{T}+\dot{\gamma}$, and

$$
H_{R}^{\prime}=P_{T}\left[\widehat{F_{R}}+\sigma(t) j^{-1} g+\left(V-V\left(\omega_{T}\right)\right) f+\left(P_{d}-P_{T}^{d}\right) j\left(E_{2}[w, w]+\dot{\gamma} f\right)\right] .
$$

Recall that $V$ is the potential defined in (2.1.3). The remainder $H_{R}^{\prime}$ can be rewritten as $H_{R}^{\prime}=H_{\mathrm{I}}^{\prime}+F_{\mathrm{II}}$, with

$$
\begin{aligned}
H_{\mathrm{I}}^{\prime}= & -P_{T}^{d} F_{\mathrm{II}}+H_{1}-P_{T}^{d} H_{1} \quad \text { and } \\
H_{1}= & \widehat{F}_{\mathrm{I}}+\sigma(t) j^{-1} g \\
& +\left(V-V\left(\omega_{T}\right)\right) f+\left(P_{d}-P_{T}^{d}\right) j\left(E_{2}[w, w]+\dot{\gamma} f\right) .
\end{aligned}
$$

LEMMA 4.1. - For any function $\alpha$ such that $\left\|\left(1+x^{2}\right) \alpha\right\|_{2}<\infty$, we have that

$$
\left\|\left(1+x^{2}\right) P_{d} \alpha\right\|_{2}=\mathcal{R}(\omega)\left\|\left(1+x^{2}\right) \alpha\right\|_{2} .
$$

PROPOSITION 4.3. - The remainder $H_{1}$ satisfies

$$
\begin{aligned}
\left\|\left(1+x^{2}\right) H_{1}\right\|_{2}= & \mathcal{R}_{1}\left(\omega,|z|+\|f\|_{\infty}\right)\left(\left|z^{3}\right|+\|f\|_{\rho}\left(|z|+\|f\|_{\infty}\right)\right) \\
& +\left(\left|\omega_{T}-\omega\right|+\left(|z|+\|f\|_{\rho}\right)^{2}\right)\left(|z|^{2}+\|f\|_{\rho}\right) .
\end{aligned}
$$

Proof. - Considering the different terms in $H_{1}$, we have

$$
\begin{aligned}
& \left\|\left(1+x^{2}\right) F_{\mathrm{I}}\right\|_{2}=\mathcal{R}\left(\omega,|z|+\|f\|_{\infty}\right)\left(|z|^{3}+\|f\|_{\rho}\left(|z|+\|f\|_{\infty}\right)\right), \\
& \left\|\left(1+x^{2}\right) \sigma(t) j g\right\|_{2}=\left|\sigma(t)\left\|\left(1+x^{2}\right) j P_{T}^{d} f\right\|_{2}=\mathcal{R}_{1}\left(\omega,|z|+\|f\|_{\infty}\right)\right| \sigma(t) \mid\|f\|_{\rho},
\end{aligned}
$$




$$
\begin{aligned}
& \left\|\left(1+x^{2}\right)\left(V-V\left(\omega_{T}\right)\right) f\right\|_{2}=\mathcal{R}_{1}\left(\omega,|z|+\|f\|_{\infty}\right)\left|\omega_{T}-\omega\right|\|f\|_{\rho}, \\
& \left\|\left(1+x^{2}\right)\left(P_{d}-P_{T}^{d}\right) j E_{2}[w, w]\right\|_{2}=\mathcal{R}_{1}\left(\omega,|z|+\|f\|_{\infty}\right)\left|\omega_{T}-\omega \| z\right|^{2}, \\
& \left\|\left(1+x^{2}\right)\left(P_{d}-P_{T}^{d}\right) j f\right\|_{2}=\mathcal{R}\left(\omega,|z|+\|f\|_{\infty}\right)\left|\omega_{T}-\omega\right|\|f\|_{\rho}, \\
& |\sigma(t)| \leqslant\left|\omega_{T}-\omega\right|+\left(|z|+\|f\|_{\rho}\right)^{2} .
\end{aligned}
$$

PROPOSITION 4.4. - The remainder $H_{\mathrm{I}}^{\prime}$ satisfies

$$
\begin{aligned}
\left\|\left(1+x^{2}\right) H_{\mathrm{I}}^{\prime}\right\|_{2}= & \mathcal{R}_{1}\left(\omega,|z|+\|f\|_{\infty}\right)\left(\left|z^{3}\right|+\|f\|_{\rho}\left(|z|+\|f\|_{\infty}\right)\right) \\
& +\left(\left|\omega_{T}-\omega\right|+\left(|z|+\|f\|_{\rho}\right)^{2}\right)\left(|z|^{2}+\|f\|_{\rho}\right) .
\end{aligned}
$$

Proof. - Notice first that the estimate (4.2.14) is also valid for $H_{1}$, and due to Lemma 4.1 for $P_{T}^{d} H_{1}$. From Section 3, we have

$$
\left\|\left(1+x^{2}\right) P_{T}^{d} F_{\mathrm{II}}\right\|_{2}=\mathcal{R}\left(\omega,|z|+\|f\|_{\infty}\right)\left(|z|^{2 r+1}+\|f\|_{\infty}^{2 r}\|f\|_{\rho}\right) .
$$

Combining these estimates, we get the proposition.

Coming back to Eq. (4.2.8) for $h$, we replace it by

$$
\dot{h}=C_{M} h-P_{T} j E_{2}[w, w]+H_{R}
$$

where

$$
C_{M}=C_{M}(t)=C_{T}+i \sigma(t)\left(P_{T}^{+}-P_{T}^{-}\right)
$$

$H_{R}=F_{\mathrm{II}}+H_{\mathrm{I}}$ and

$$
H_{\mathrm{I}}=H_{\mathrm{I}}^{\prime}+\sigma(t)\left[P_{T} j^{-1}-i\left(P_{T}^{+}-P_{T}^{-}\right)\right] h .
$$

PROPOSITION 4.5. - In the remainder $H_{R}=H_{\mathrm{I}}+F_{\mathrm{II}}$, the term $H_{\mathrm{I}}$ satisfies the same estimate (4.2.14) as $H_{\mathrm{I}}^{\prime}$, that is:

$$
\begin{aligned}
\left\|\left(1+x^{2}\right) H_{\mathrm{I}}\right\|_{2}= & \mathcal{R}_{1}\left(\omega,|z|+\|f\|_{\infty}\right)\left(|z|^{3}+\left(|z|+\|f\|_{\infty}\right)\|f\|_{\rho}\right. \\
& \left.+\left(|\omega(T)-\omega|+\left(|z|+\|f\|_{\rho}\right)^{2}\right)\left(|z|^{2}+\|f\|_{\rho}\right)\right) .
\end{aligned}
$$

For the proof, notice that

$$
\left\|\left(1+x^{2}\right)\left(P_{T} j^{-1}-i\left(P_{T}^{+}-P_{T}^{-}\right)\right) h\right\|_{2}=\mathcal{R}\left(\omega_{T}\right)\|h\|_{\rho}=\mathcal{R}_{1}(\omega)\|f\|_{\rho} .
$$

\subsubsection{Further decomposition}

We rewrite (4.2.16) in a more detailed form as

$$
\dot{h}=C_{M} h+H_{20} z^{2}+H_{11} z \bar{z}+H_{02} \bar{z}^{2}+H_{R} .
$$

Here, the coefficients $H_{i j}$ are defined by

$$
H_{20}=-P_{T} j E_{2}[u, u], \quad H_{11}=-2 P_{T} j E_{2}\left[u, u^{*}\right], \quad H_{02}=-P_{T} j E_{2}\left[u^{*}, u^{*}\right] .
$$


We now introduce a new function $h_{1}$ defined by

$$
h=h_{1}+k+k_{1}
$$

where

$$
\begin{aligned}
& k=a_{20} z^{2}+a_{11} z \bar{z}+a_{02} \bar{z}^{2}, \quad k_{0}=k_{\mid t=0}, \\
& k_{1}=-\exp \left(\int_{0}^{t} C_{M}(\tau) d \tau\right) k_{0}
\end{aligned}
$$

with some $a_{i j} \equiv a_{i j}(\omega, x)$ satisfying $a_{i j}=\bar{a}_{j i}$. The purpose here is to extract from $f$ the contribution which is quadratic in $z$. Note that $h_{10}=h_{1}(t=0)=h_{0}$. We look for coefficients $a_{i j}$ such that the resulting equation for $h_{1}$ has the form

$$
\dot{h}_{1}=C_{M}(t) h_{1}+\widehat{H_{R}}
$$

with an appropriate estimate for $\widehat{H_{R}}$.

Substituting (4.2.24) into (4.2.21), we get

$$
\begin{aligned}
\dot{h}_{1}= & \dot{h}-\left(a_{20 \omega} z^{2}+a_{11 \omega} z \bar{z}+a_{02 \omega} \bar{z}^{2}\right) \dot{\omega}-\left(2 a_{20} z+a_{11} \bar{z}\right) \dot{z}-\left(a_{11} z+2 a_{02} \bar{z}\right) \dot{\bar{z}}-\dot{k}_{1} \\
= & {\left[C_{T}+i \sigma\left(P_{T}^{+}-P_{T}^{-}\right)\right] h+H_{02} z^{2}+H_{11} z \bar{z}+H_{02} \bar{z}^{2}+H_{R} } \\
& -\left(a_{20 \omega} z^{2}+a_{11 \omega} z \bar{z}+a_{02 \omega} \bar{z}^{2}\right) \mathcal{R}\left(\omega,|z|+\|f\|_{\rho}\right)\left(|z|+\|f\|_{\rho}\right)^{2} \\
& -\left(2 a_{20} z+a_{11} \bar{z}\right)\left(i \mu z+\mathcal{R}\left(\omega,|z|+\|f\|_{\rho}\right)\left(|z|+\|f\|_{\rho}\right)^{2}\right) \\
& -\left(a_{11} z+2 a_{02} \bar{z}\right)\left(-i \mu \bar{z}+\mathcal{R}\left(\omega,|z|+\|f\|_{\rho}\right)\left(|z|+\|f\|_{\rho}\right)^{2}\right)-C_{M} k_{1} \\
= & {\left[C_{T}+i \sigma(t)\left(P_{T}^{+}-P_{T}^{-}\right)\right]\left(h-k-k_{1}\right)+\widehat{H_{R}}, }
\end{aligned}
$$

where we have used that $\dot{\omega}=\mathcal{R}\left(\omega,|z|+\|f\|_{\infty}\right)\left(|z|+\|f\|_{\rho}\right)^{2}$ and $\dot{z}-i \mu z=\mathcal{R}(\omega,|z|+$ $\left.\|f\|_{\infty}\right)\left(|z|+\|f\|_{\rho}\right)^{2}$. It is convenient to replace in (4.2.27) $\mu(\omega)$ by $\mu_{T}=\mu(\omega(T))$ and include the correction in the remainder. This will avoid later the differentiation of $\left(C_{T}-2 i \mu_{T}\right)^{-1}$ with respect to $t$.

Equating the coefficients of the quadratic powers of $z$, we get

$$
\begin{aligned}
H_{20}-2 i \mu_{T} a_{20} & =-C_{T} a_{20}, \\
H_{11} & =-C_{T} a_{11}, \\
H_{02}+2 i \mu_{T} a_{02} & =-C_{T} a_{02}
\end{aligned}
$$

and

$$
\widehat{H_{R}}=H_{R}+H_{\mathrm{II}}
$$

where $H_{\text {II }}$ is defined as

$$
\begin{aligned}
H_{\mathrm{II}}= & \sum a_{i j \omega} \mathcal{R}(\omega)|z|^{2}\left(|z|+\|f\|_{\rho}\right)^{2}+\sum a_{i j} \mathcal{R}(\omega)|z|\left(|z|+\|f\|_{\rho}\right)^{2} \\
& +\sum a_{i j} \mathcal{R}(\omega)|z|^{2}\left|\mu_{T}-\mu\right|-i \sigma\left(P_{T}^{+}-P_{T}^{-}\right) k .
\end{aligned}
$$


The dependency in $x$ appears here through the coefficients $a_{i j}=a_{i j}(\omega, x)$. Notice that $H_{i j} \in X_{T}^{c}$ are smooth, exponentially vanishing functions at infinity. Let us solve for $a_{11}$ as

$$
a_{11}=-C_{T}^{-1} H_{11} .
$$

The function $a_{11}$ is also smooth and exponentially decreasing at infinity. Furthermore, we have to invert $C_{T} \pm 2 i \mu$, with both points $\pm 2 i \mu$ in the continuous spectrum of $C_{T}$. It implies that, in general, the functions

$$
a_{20}=-\left(C_{T}-2 i \mu_{T}\right)^{-1} H_{20}, \quad \text { and } \quad a_{02}=\bar{a}_{20}=-\left(C_{T}+2 i \mu_{T}\right)^{-1} H_{02}
$$

do not decrease at infinity. They behave like solutions of the homogeneous equation $\left(C-2 i \mu_{T}\right) a=0$, and thus oscillate at infinity. Nevertheless, there exists a special choice for these inverse operators that leads to preferable properties of $h_{1}$. This choice is

$$
a_{20}=-\left(C_{T}-2 i \mu_{T}-0\right)^{-1} H_{20}, \quad a_{02}=\bar{a}_{20}=-\left(C_{T}+2 i \mu_{T}-0\right)^{-1} H_{02} .
$$

The property is reflected in Lemma 5.2 that claims that for $t \geqslant 0$,

$$
\begin{aligned}
& \left\|\mathrm{e}^{C_{T} t}\left(C_{T} \pm 2 i \mu_{T}-0\right)^{-1} P_{T}^{ \pm} h\right\|_{\rho} \\
& \quad \leqslant c(1+t)^{-3 / 2}\left(\|h\|_{2}+\left\|\left(1+x^{2}\right)^{3 / 2} h\right\|_{1}\right) .
\end{aligned}
$$

It corresponds to the classical fact that $(i \lambda-0)^{-1} \mathrm{e}^{i \lambda t} \rightarrow 0$ as $t \rightarrow \infty$, in the sense of distributions. The weight $\rho(x)=\left(1+x^{2}\right)^{-\alpha}$ here must satisfy the stronger condition $\alpha \geqslant 2$ (see Lemma 5.2).

The remainder $H_{\text {II }}$ can be written as

$$
\begin{aligned}
H_{\mathrm{II}}= & \sum a_{i j \omega} \mathcal{R}(\omega)|z|^{2}\left(|z|+\|f\|_{\rho}\right)^{2} \\
& +\sum a_{i j} \mathcal{R}(\omega)|z|\left[\left(|z|+\|f\|_{\rho}\right)^{2}+|z|\left|\omega_{T}-\omega\right|\right] .
\end{aligned}
$$

More explicitly, it takes the form

$$
H_{\mathrm{II}}=\sum_{m, n}\left(C_{T}-2 i \mu_{T} m-0\right)^{-1} P_{T}^{n} \mathcal{A}_{m n}
$$

with $m \in\{-1,0,1\}$ and $n \in\{+,-\}$.

Proposition 4.6. - The coefficients $\mathcal{A}_{m n}$ that appear in (4.2.36) satisfy the estimate:

$$
\begin{aligned}
\left\|\mathcal{A}_{m n}\right\|_{2}+\left\|\left(1+x^{2}\right)^{3 / 2} \mathcal{A}_{m n}\right\|_{1}= & \mathcal{R}\left(\omega,|z|+\|f\|_{\rho}\right)|z|\left(|z|\left|\omega_{T}-\omega\right|\right. \\
& \left.+\left(|z|+\|f\|_{\rho}\right)^{2}\right) .
\end{aligned}
$$

\subsection{Transformation of the equation for $z$}

Finally, let us consider Eq. (3.2.9) for $z$. We first replace the terms containing $f$ by their local leading order where $f$ is replaced by $k$. Indeed, $k$ is also the leading order compared to $k_{1}$. This follows from (4.2.34) that claims that, for example in $L_{2}(\rho)$,

$$
\left\|k_{1}\right\|_{\rho} \leqslant c\left|z_{0}\right|^{2} \frac{1}{(1+t)^{3 / 2}} .
$$


On the other hand, $k$ is of order $|z|^{2}$. We will see later that if we assume that $\left|z_{0}\right|=\varepsilon^{1 / 2}$, then $|z|^{2}=\frac{C \varepsilon}{1+\varepsilon t}$. We will see that a relatively weak smallness of $k_{1}$ compared to $k$ will be sufficient to neglect it in the leading terms of the equation for $z$.

$$
\left\|k_{1}\right\|_{\rho} \leqslant c \frac{\varepsilon}{(1+t)^{3 / 2}} \leqslant c \frac{\varepsilon}{1+\varepsilon t} \frac{1}{\sqrt{1+t}} .
$$

We write (3.2.9) in the form

$$
\begin{aligned}
\dot{z}= & i\left(\mu z+Z_{20} z^{2}+Z_{11} z \bar{z}+Z_{02} \bar{z}^{2}+Z_{30} z^{3}+Z_{21} z^{2} \bar{z}+Z_{12} z \bar{z}^{2}+Z_{03} \bar{z}^{3}\right) \\
& +i\left(Z_{30}^{\prime} z^{3}+Z_{21}^{\prime} z^{2} \bar{z}+Z_{12}^{\prime} z \bar{z}^{2}+Z_{03}^{\prime} \bar{z}^{3}\right)+\widetilde{Z_{R}}
\end{aligned}
$$

where the coefficients $Z_{i j}$ are defined given in (4.1.5) are real. We have

$$
\begin{array}{ll}
i Z_{30}^{\prime}=\left\langle a_{20}, Z_{10}^{\prime}\right\rangle, & i Z_{21}^{\prime}=\left\langle a_{11}, Z_{10}^{\prime}\right\rangle+\left\langle a_{20}, Z_{01}^{\prime}\right\rangle, \\
i Z_{03}^{\prime}=\left\langle a_{02}, Z_{01}^{\prime}\right\rangle, & i Z_{12}^{\prime}=\left\langle a_{02}, Z_{10}^{\prime}\right\rangle+\left\langle a_{11}, Z_{01}^{\prime}\right\rangle .
\end{array}
$$

We are specially interested in the coefficient $Z_{21}^{\prime}$, which is given by the formula

$$
\begin{aligned}
i Z_{21}^{\prime}= & -\left\langle C_{T}^{-1} 2 P_{T} j E_{2}\left[u, u^{*}\right], 2 \frac{E_{2}\left[u^{*}, u\right]}{\langle u, j u\rangle}\right\rangle \\
& -\left\langle\left(C_{T}-2 i \mu_{T}-0\right)^{-1} P_{T} j E_{2}[u, u], 2 \frac{E_{2}[u, u]}{\langle u, j u\rangle}\right\rangle .
\end{aligned}
$$

Proposition 4.7. - Suppose that the non-degeneracy condition $\mid\left\langle E_{2}[u, u]\right.$, $u(2 i \mu)\rangle\left.\right|^{2} \neq 0$ is satisfied, then

$$
\operatorname{Re} i Z_{21}^{\prime}<0 .
$$

Proof. - We first notice that the coefficient $\left\langle C_{T}^{-1} P_{T} j E_{2}\left[u, u^{*}\right], E_{2}\left[u, u^{*}\right]\right\rangle$ that appears in the expression (4.3.5) for $i Z_{21}^{\prime}$ is real. Indeed, it is easy to check that $E_{2}\left[u, u^{*}\right]$ is real, and all the operators in the above scalar product are also real. Recall also that from Proposition $4.1\langle u, j u\rangle=i \delta$, with $\delta>0$. It follows that $\operatorname{Re} i Z_{21}^{\prime}$ reduces to

$$
\begin{aligned}
\operatorname{Re} i Z_{21}^{\prime} & =-\operatorname{Re} 2 \frac{\left\langle\left(C_{T}-2 i \mu_{T}-0\right)^{-1} P_{T} j E_{2}[u, u], E_{2}[u, u]\right\rangle}{\langle u, j u\rangle} \\
& =-\frac{2}{\delta} \operatorname{Im}\left\langle\left(C_{T}-2 i \mu_{T}-0\right)^{-1} P_{T} j E_{2}[u, u], E_{2}[u, u]\right\rangle .
\end{aligned}
$$

Using that $P_{T}$ commutes with $C_{T}^{-1}$, we have $C_{T}^{-1} P_{T}=P_{T} C_{T}^{-1} P_{T}$. We have also that $P_{T}^{*}=j^{-1} P_{T} j$. It follows that

$$
\operatorname{Re} i Z_{21}^{\prime}=-\frac{2}{\delta} \operatorname{Im}\left\langle\left(C_{T}-2 i \mu_{T}-0\right)^{-1} \alpha, j \alpha\right\rangle
$$

with $\alpha=P_{T} j E_{2}[u, u]$.

From the spectral representation (2.1.9), we have for $\alpha \in X_{T}$,

$$
\left\langle\left(C_{T}-2 i \mu-0\right)^{-1} \alpha, j \alpha\right\rangle
$$




$$
\begin{aligned}
& =\frac{1}{i} \int_{\omega}^{\infty} \theta(\lambda) d \lambda\left(\frac{\langle u(i \lambda), j \alpha\rangle \overline{\langle u(i \lambda), j \alpha\rangle}}{i \lambda-2 i \mu-0}+\frac{\left\langle u^{*}(i \lambda), j \alpha\right\rangle \overline{\left\langle u^{*}(i \lambda), j \alpha\right\rangle}}{-i \lambda-2 i \mu-0}\right) \\
& =-\int_{\omega}^{\infty} \theta(\lambda) d \lambda\left(\frac{\langle u(i \lambda), j \alpha\rangle \overline{\langle u(i \lambda), j \alpha\rangle}}{\lambda-2 \mu+i 0}-\frac{\left(u^{*}(i \lambda), j \alpha\right)\left\langle\overline{\left.u^{*}(i \lambda), j \alpha\right\rangle}\right.}{\lambda+2 \mu-i 0}\right) .
\end{aligned}
$$

Using that

$$
\frac{1}{\lambda+i 0}=\operatorname{p} \cdot \mathrm{v} \cdot \frac{1}{\lambda}-i \pi \delta(\lambda)
$$

where p.v. is the Cauchy principal value and $\delta$ the Dirac distribution, we have

$$
\begin{aligned}
& \left\langle\left(C_{T}-2 i \mu-0\right)^{-1} \alpha, j \alpha\right\rangle \\
& =-\int_{\omega}^{\infty} \theta(\lambda) d \lambda\left(\frac{\langle u(i \lambda), j \alpha\rangle \overline{\langle u(i \lambda), j \alpha\rangle}}{\lambda-2 \mu}-\frac{\left\langle u^{*}(i \lambda), j \alpha\right\rangle \overline{\left\langle u^{*}(i \lambda), j \alpha\right\rangle}}{\lambda+2 \mu}\right) \\
& \quad+i \pi \theta(2 \mu)\langle u(i 2 \mu), j \alpha\rangle \overline{\langle u(i 2 \mu), j \alpha\rangle} .
\end{aligned}
$$

The integral term in (4.3.11) is real. Thus,

$$
\operatorname{Im}\left\langle\left(C_{T}-2 i \mu-0\right)^{-1} \alpha, j \alpha\right\rangle=\operatorname{Im}(i \pi \theta(2 \mu))|\langle u(2 i \mu), j \alpha\rangle|^{2},
$$

with

$$
\theta(2 \mu)=\frac{1}{2 \pi \sqrt{2 \mu-\omega} N^{2}(2 \mu)} .
$$

Assuming the non-degeneracy condition that $\left|\left\langle E_{2}[u, u], u(2 i \mu)\right\rangle\right|^{2} \neq 0$, we get

$$
\operatorname{Re} i Z_{21}^{\prime}=-\frac{2}{\delta} \pi \theta(2 \mu)\left|\left\langle E_{2}[u, u], u(2 i \mu)\right\rangle\right|^{2}<0 \text {. }
$$

We now need an estimate on the remainder $\widetilde{Z_{R}}$.

PROPOSITION 4.8. - The remainder $\widetilde{Z_{R}}$ has the form

$$
\widetilde{Z_{R}}=Z_{R}+|z| \mathcal{R}_{1}(\omega)\left(|\omega(T)-\omega|\|h\|_{\rho}+\left\|h_{1}\right\|_{\rho}\right)
$$

where $Z_{R}$ satisfies estimate (3.2.10).

Proof. - The remainder $\widetilde{Z_{R}}$ is given by

$$
\widetilde{Z_{R}}=Z_{R}+z\left\langle f-k, Z_{10}^{\prime}\right\rangle+\bar{z}\left\langle f-k, Z_{01}^{\prime}\right\rangle .
$$

Since $f-k=g+k_{1}+h_{1}$, we have

$$
\begin{aligned}
\left|\left\langle f-k, Z_{10}^{\prime}\right\rangle\right| & \leqslant \mathcal{R}(\omega)\left(\|g\|_{\rho}+\left\|k_{1}\right\|_{\rho}+\left\|h_{1}\right\|_{\rho}\right) \\
& \leqslant \mathcal{R}_{1}(\omega)\left(\left|\omega_{T}-\omega\right|\|h\|_{\rho}+\left\|k_{1}\right\|_{\rho}+\left\|h_{1}\right\|_{\rho}\right) .
\end{aligned}
$$

Therefore 


$$
\begin{aligned}
\widetilde{Z_{R}}= & Z_{R}+\mathcal{R}_{1}(\omega)|z|\left[\left|\omega_{T}-\omega\right|\|h\|_{\rho}+\left\|k_{1}\right\|_{\rho}+\left\|h_{1}\right\|_{\rho}\right] \\
= & \mathcal{R}_{1}\left(\omega,|z|+\|f\|_{\infty}\right)\left[\left(|z|^{2}+\|f\|_{\rho}\right)^{2}+\mid z \| \omega_{T}\right. \\
& \left.-\omega\left|\|h\|_{\rho}+\right| z\left|\left\|k_{1}\right\|_{\rho}+\right| z \mid\left\|h_{1}\right\|_{\rho}\right] .
\end{aligned}
$$

We can apply now the method of normal coordinates to (4.3.3).

PROPOSITION 4.9. - There exist coefficients $c_{i j}$ such that the new function $z_{1}$ defined by

$$
z_{1}=z+c_{20} z^{2}+c_{11} z \bar{z}+c_{02} \bar{z}^{2}+c_{30} z^{3}+c_{12} z \bar{z}^{2}+c_{03} \bar{z}^{3},
$$

satisfies an equation of the form

$$
\dot{z_{1}}=i \mu(\omega) z_{1}+i K(\omega)\left|z_{1}\right|^{2} z_{1}+\widehat{Z_{R}}
$$

where $\widehat{Z_{R}}$ satisfies estimates of the same type as $\widetilde{Z_{R}}$, and

$$
\operatorname{Re} i K=\operatorname{Re} i Z_{21}^{\prime}<0 .
$$

Proof. - Substituting $z_{1}$ in Eq. (3.2.9) for $z$, we get

$$
\begin{aligned}
\dot{z}_{1}= & \left(c_{20 \omega} z^{2}+c_{11 \omega} z \bar{z}+c_{02 \omega} \bar{z}^{2}+c_{30} \omega z^{3}+c_{12 \omega} z \bar{z}^{2}+c_{03 \omega} \bar{z}^{3}\right) \dot{\omega} \\
& +\left(1+2 c_{20} z+c_{11} \bar{z}+3 c_{30} z^{2}+c_{12} \bar{z}^{2}\right) \dot{z}+\left(c_{11} z+2 c_{02} \bar{z}+2 c_{12} z \bar{z}+c_{03} \bar{z}^{2}\right) \dot{\bar{z}} \\
= & \mathcal{R}\left(\omega,|z|+\|f\|_{\infty}\right)\left(|z|+\|f\|_{\rho}\right)^{2}+i\left(\mu z+Z_{20} z^{2}+Z_{11} z \bar{z}+Z_{02} \bar{z}^{2}+Z_{30} z^{3}\right. \\
& \left.+Z_{21} z^{2} \bar{z}+Z_{12} z \bar{z}^{2}+Z_{03} \bar{z}^{3}+Z_{21}^{\prime} z^{2} \bar{z}+Z_{12}^{\prime} z \bar{z}^{2}+Z_{30}^{\prime} z^{3}+Z_{03}^{\prime} \bar{z}^{3}+\widetilde{Z}_{R}\right) \\
& +\left(2 c_{20} z+c_{1} \bar{z}\right) i\left(\mu z+Z_{20} z^{2}+Z_{11} z \bar{z}+Z_{02} \bar{z}^{2}+\mathcal{R}(\omega)|z|^{3}+\widetilde{Z}_{R}\right) \\
& +\left(3 c_{30} z^{2}+c_{12} \bar{z}^{2}\right)\left(i \mu z+\mathcal{R}(\omega)|z|^{2}+\widetilde{Z}_{R}\right) \\
& +\left(c_{11} z+2 c_{02} \bar{z}\right)(-i)\left(\mu \bar{z}+\bar{Z}_{20} \bar{z}^{2}+\bar{Z}_{11} z \bar{z}+\bar{Z}_{02} z^{2}+\mathcal{R}(\omega)|z|^{3}+\widetilde{Z}_{R}\right) \\
& +\left(3 c_{03} \bar{z}^{2}+c_{21} z^{2}\right)(-i)\left(\mu z+\mathcal{R}(\omega)|z|^{2}+\widetilde{\bar{Z}}_{R}\right) \\
= & i \mu\left(z+c_{20} z^{2}+c_{11} z \overline{+} c_{02} \bar{z}^{2}+c_{30} z^{3}+c_{12} z \bar{z}^{2}+c_{03} \bar{z}^{3}\right) \\
& +i K \bar{z} z^{2}+\mathcal{R}(\omega)|z|^{4}+\widehat{Z}_{R} .
\end{aligned}
$$

Equating the coefficients, we get

$$
\begin{aligned}
& c_{20}=-\frac{1}{\mu} Z_{20}, \quad c_{11}=\frac{1}{\mu} Z_{11}, \quad c_{02}=\frac{1}{3 \mu} Z_{02}, \\
& i Z_{21}+i Z_{21}^{\prime}+i c_{11} Z_{20}+2 i c_{20} Z_{11}-i c_{11} \bar{Z}_{11}-2 i c_{02} \bar{Z}_{02}=i K .
\end{aligned}
$$

The remainder $\widehat{Z}_{R}$ is defined by

$$
\begin{aligned}
\widehat{Z}_{R}= & \mathcal{R}\left(\omega,|z|+\|f\|_{\infty}\right)|z|^{2}\left(|z|+\|f\|_{\rho}\right)^{2}+\widetilde{Z}_{R} \\
= & \mathcal{R}_{1}\left(\omega,|z|+\|f\|_{\infty}\right)\left[\left(|z|^{2}+\|f\|_{\rho}\right)^{2}+\mid z \| \omega_{T}\right. \\
& \left.-\omega\left|\|h\|_{\rho}+\right| z \mid\left(\left\|k_{1}\right\|_{\rho}+\left\|h_{1}\right\|_{\rho}\right)\right] .
\end{aligned}
$$


Denoting $K_{T}=K\left(\omega_{T}\right)$, the equation for $z_{1}$ is rewritten as

$$
\dot{z_{1}}=i \mu z_{1}+i K_{T}\left|z_{1}\right|^{2} z_{1}+\widehat{\widehat{Z_{R}}}
$$

where

$$
\begin{aligned}
\widehat{\widehat{Z_{R}}}= & \widehat{Z_{R}}+\mathcal{R}_{1}\left(\omega,|z|+\|f\|_{\infty}\right)|z|^{3}\left|\omega_{T}-\omega\right| \\
= & \mathcal{R}_{1}\left(\omega,|z|+\|f\|_{\infty}\right)\left[\left(|z|^{2}+\|f\|_{\rho}\right)^{2}+|z|\left(\left\|k_{1}\right\|_{\rho}+\left\|h_{1}\right\|_{\rho}\right)\right. \\
& \left.+|z|\left|\omega_{T}-\omega\right|\left(|z|^{2}+\|h\|_{\rho}\right)\right] .
\end{aligned}
$$

It is easier to deal with $y=\left|z_{1}\right|^{2}$ rather that $z_{1}$ because $y$ decreases at infinity while $z_{1}$ is oscillating. The equation satisfied by $y$ is simply obtained by multiplying (4.3.20) by $z_{1}$ and taking the real part:

$$
\dot{y}=\operatorname{Re}\left(i K_{T}\right) y^{2}+Y_{R},
$$

where

$$
\begin{aligned}
Y_{R}= & \mathcal{R}_{1}\left(\omega,|z|+\|f\|_{\infty}\right)|z|\left[\left(|z|^{2}+\|f\|_{\rho}\right)^{2}+|z|\left(\left\|k_{1}\right\|_{\rho}+\left\|h_{1}\right\|_{\rho}\right)\right. \\
& \left.+|z|\left|\omega_{T}-\omega\right|\left(|z|^{2}+\|h\|_{\rho}\right)\right] .
\end{aligned}
$$

\subsection{Summary of transformed equations}

We summarize the main formulas of Sections 4.1-4.3. The first equation is the evolution of $\omega_{1}$ given in Proposition 4.1:

$$
\dot{\omega}_{1}=\widehat{\Omega}_{R},
$$

where

$$
\widehat{\Omega}_{R}=\mathcal{R}\left(\omega,|z|+\|f\|_{\infty}\right)\left(|z|^{2}+\|f\|_{\rho}\right)^{2},
$$

and $\omega_{1}$ and $\omega$ are related by (see (4.1.6))

$$
\omega_{1}-\omega=\mathcal{R}(\omega)|z|\left(|z|+\|f\|_{\rho}\right) .
$$

The second equation describes the evolution of $z_{1}$ (see (4.3.20))

$$
\dot{z_{1}}=i \mu(\omega) z_{1}+i K_{T}\left|z_{1}\right|^{2} z_{1}+\widehat{\widehat{Z_{R}}}
$$

with estimate (4.3.26) for $\widehat{Z_{R}}$. From (4.3.19), $z$ and $z_{1}$ are related by that

$$
z_{1}-z=\mathcal{R}(\omega)|z|^{2}
$$

The third equation is the evolution of $y=\left|z_{1}\right|^{2}$

$$
\dot{y}=\operatorname{Re}\left(i K_{T}\right) y^{2}+Y_{R},
$$


where

$$
\left|Y_{R}\right| \leqslant\left|z_{1}\right|\left|\widehat{\widehat{Z_{R}}}\right| \text {. }
$$

The negativity of $\operatorname{Re}\left(i K_{T}\right)$ is a key point in the analysis and was proved in Proposition 4.7. As for the initial data, we will discuss them in Section 4.5.3.

We need also two additional equations for $h$ and $h_{1}$. We recall that

$$
f=g+h, \quad g=P_{T}^{d} f, \quad h=P_{T} f,
$$

and $h$ is decomposed into

$$
h=k+k_{1}+h_{1}
$$

where $k$ and $h_{1}$ are defined in (4.2.24)-(4.2.25). The equations satisfied by $h$ and $h_{1}$ are respectively (see (4.2.21) and (4.2.26))

$$
\begin{aligned}
& \dot{h}=C_{M}(t) h-P_{T} j E_{2}[w, w]+H_{R}, \\
& \dot{h}_{1}=C_{M}(t) h_{1}+\widehat{H_{R}} .
\end{aligned}
$$

The operator $C_{M}$ is defined in (4.2.17), $H_{R}=H_{\mathrm{I}}+F_{\mathrm{II}}$ and $\widehat{H_{R}}=H_{R}+H_{\mathrm{II}}$. The remainders $F_{\mathrm{II}}$ and $H_{\mathrm{I}}$ are estimated in (3.3.31) and (4.2.19) respectively, and, $H_{\mathrm{II}}$ is estimated in (4.2.36) and (4.2.37).

\subsection{Estimates of remainders and initial conditions}

In the next parts, we will study majorants for four functions (see Section 5.1) $\left|\omega_{T}-\omega\right|$, $|z|,\|f\|_{\infty}$, and $\left\|h_{1}\right\|_{\rho}$. For this purpose we need to estimate these quantities in terms of $\dot{\omega}_{1}, z_{1}, h$ and $h_{1}$. Also, we need to estimate the remainders in the transformed equations in terms of $\left|\omega_{T}-\omega\right|,|z|,\|f\|_{\infty}$, and $\left\|h_{1}\right\|_{\rho}$. This is the purpose of this section. This will allow us to obtain later a closed system of equations for the majorants. We will also state the hypothesis on initial conditions, and control the initial conditions for $z_{1}, h$ and $h_{1}$.

\subsubsection{Bounds for $\left|\omega_{T}-\omega\right|,\|h\|_{\rho},\|f\|_{\rho}$ and $|z|$}

We have

$$
\begin{aligned}
& \left|\omega_{T}-\omega\right| \leqslant\left|\omega_{1 T}-\omega_{1}\right|+\left|\omega_{1 T}-\omega_{T}\right|+\left|\omega_{1}-\omega\right| \\
& \leqslant \int_{t}^{T}\left|\dot{\omega}_{1}\right| d \tau+\mathcal{R}\left(\omega_{T},\left|z_{T}\right|+\left\|f_{T}\right\|_{\infty}\right)\left(\left|z_{T}\right|^{2}+\left|z_{T}\right|\left\|f_{T}\right\|_{\rho}\right) \\
& \quad+\mathcal{R}\left(\omega,|z|+\|f\|_{\infty}\right)\left(|z|^{2}+|z|\|f\|_{\rho}\right) \\
& \leqslant \max _{0 \leqslant t \leqslant T} \mathcal{R}\left(\omega,|z|+\|f\|_{\infty}\right) \\
& \quad \times\left[\int_{t}^{T}\left(|z|^{2}+\|f\|_{\rho}\right)^{2} d \tau+\left(\left|z_{T}\right|+\left\|f_{T}\right\|_{\rho}\right)^{2}+\left(|z|+\|f\|_{\rho}\right)^{2}\right] .
\end{aligned}
$$

Assuming that $\omega_{0}$ is fixed and using that

$$
|\omega| \leqslant\left|\omega_{0}\right|+\left|\omega_{0}-\omega_{T}\right|+\left|\omega-\omega_{T}\right|
$$


we have

$$
\max _{0 \leqslant t \leqslant T} \mathcal{R}\left(\omega,|z|+\|f\|_{\infty}\right)=\mathcal{R}\left(\max _{0 \leqslant t \leqslant T}\left|\omega-\omega_{T}\right|, \max _{0 \leqslant t \leqslant T}\left(|z|+\|f\|_{\infty}\right)\right) .
$$

Such quantities will be denoted by the symbol $\mathcal{R}_{2}\left(\omega,|z|+\|f\|_{\infty}\right)$. Using this notation, $\left|\omega_{T}-\omega\right|=\mathcal{R}_{2}\left(\omega,|z|+\|f\|_{\infty}\right)\left[\int_{t}^{T}\left(|z|^{2}+\|f\|_{\rho}\right)^{2} d \tau+\left(\left|z_{T}\right|+\left\|f_{T}\right\|_{\rho}\right)^{2}+\left(|z|+\|f\|_{\rho}\right)^{2}\right]$.

Turning to the function $f$, we have

$$
\|f\|_{\rho}=\mathcal{R}_{2}\left(\omega,|z|+\|f\|_{\infty}\right)\|h\|_{\rho} .
$$

Also, from (4.2.23), $h=k+k_{1}+h_{1}$, therefore

$$
\|h\|_{\rho}=\mathcal{R}(\omega)\left(\left\|k_{1}\right\|_{\rho}+|z|^{2}+\left\|h_{1}\right\|_{\rho}\right) .
$$

It implies that

$$
\|f\|_{\rho}=\mathcal{R}_{2}\left(\omega,|z|+\|f\|_{\infty}\right)\left(\left\|k_{1}\right\|_{\rho}+|z|^{2}+\left\|h_{1}\right\|_{\rho}\right)
$$

and also that

$$
|z|^{2}+\|f\|_{\rho}=\mathcal{R}_{2}\left(\omega,|z|+\|f\|_{\infty}\right)\left(\left\|k_{1}\right\|_{\rho}+|z|^{2}+\left\|h_{1}\right\|_{\rho}\right),
$$

and

$$
|z|+\|f\|_{\rho}=\mathcal{R}_{2}\left(\omega,|z|+\|f\|_{\infty}\right)\left(\left\|k_{1}\right\|_{\rho}+|z|+\left\|h_{1}\right\|_{\rho}\right) .
$$

It is obvious also that

$$
\|g\|_{\infty}=\mathcal{R}_{1}(\omega)\left|\omega_{T}-\omega\right|\|h\|_{\rho}
$$

and

$$
\begin{aligned}
\|f\|_{\infty} & \leqslant\|h\|_{\infty}+\mathcal{R}_{1}(\omega)\left|\omega_{T}-\omega\right|\|h\|_{\rho} \\
& \leqslant\|h\|_{\infty}+\mathcal{R}_{1}(\omega)\left|\omega_{T}-\omega\right|\left(\left\|k_{1}\right\|_{\rho}+|z|^{2}+\left\|h_{1}\right\|_{\rho}\right) .
\end{aligned}
$$

We will use these estimates to evaluate the right-hand side of (4.5.1). For $z$ we have

$$
\begin{aligned}
& |z| \leqslant\left|z_{1}\right|+\mathcal{R}(\omega,|z|)|z|^{2}, \quad|z|^{2} \leqslant y+\mathcal{R}(\omega,|z|)|z|^{3}, \\
& y \leqslant\left|z_{1}\right|^{2}+\mathcal{R}(\omega,|z|)|z|^{3} .
\end{aligned}
$$

\subsubsection{Remainders}

Consider the remainder $\widehat{\bar{Z}_{R}}$ in Eq. (4.3.25) for $z_{1}$. It satisfies

$$
\begin{aligned}
\left|\widehat{\widehat{Z_{R}}}\right|= & \mathcal{R}_{2}\left(\omega,|z|+\|f\|_{\infty}\right)\left[\left(|z|^{2}+\|f\|_{\rho}\right)^{2}\right. \\
& \left.+|z|\left(\left\|k_{1}\right\|_{\rho}+\left\|h_{1}\right\|_{\rho}+\left|\omega_{T}-\omega\right|\left(|z|^{2}+\|h\|_{\rho}\right)\right)\right]
\end{aligned}
$$




$$
\begin{aligned}
= & \mathcal{R}_{2}\left(\omega,|z|+\|f\|_{\infty}\right)\left[\left(\left\|k_{1}\right\|_{\rho}+|z|^{2}+\left\|h_{1}\right\|_{\rho}\right)^{2}\right. \\
& \left.+|z|\left(\left\|k_{1}\right\|_{\rho}+\left\|h_{1}\right\|_{\rho}+\left|\omega_{T}-\omega\right|\left(\left\|k_{1}\right\|_{\rho}+|z|^{2}+\left\|h_{1}\right\|_{\rho}\right)\right)\right] \\
= & \mathcal{R}_{2}\left(\omega,|z|+\|f\|_{\infty}\right)\left[\left(\left\|k_{1}\right\|_{\rho}+|z|^{2}+\left\|h_{1}\right\|_{\rho}\right)\right. \\
& \left.\times\left(\left\|k_{1}\right\|_{\rho}+|z|^{2}+\left\|h_{1}\right\|_{\rho}+|z|\left|\omega_{T}-\omega\right|\right)+|z|\left(\left\|k_{1}\right\|_{\rho}+\left\|h_{1}\right\|_{\rho}\right)\right] .
\end{aligned}
$$

In Eq. (4.4.6) for $y$, the remainder $Y_{R}$ satisfies

$$
\begin{aligned}
\left|Y_{R}\right|= & \mathcal{R}_{2}\left(\omega,|z|+\|f\|_{\infty}\right)|z|\left[\left(\left\|k_{1}\right\|_{\rho}+|z|^{2}+\left\|h_{1}\right\|_{\rho}\right)\right. \\
& \left.\times\left(\left\|k_{1}\right\|_{\rho}+|z|^{2}+\left\|h_{1}\right\|_{\rho}+z\left|\omega_{T}-\omega\right|\right)+|z|\left(\left\|k_{1}\right\|_{\rho}+\left\|h_{1}\right\|_{\rho}\right)\right] .
\end{aligned}
$$

Consider now the remainder $H_{R}$ in Eq. (4.4.10) for $h$. We recall that $H_{R}=H_{\mathrm{I}}+F_{\mathrm{II}}$ where $H_{\mathrm{I}}$ and $F_{\mathrm{II}}$ are given and estimated in (3.3.31) and (4.2.19). The estimate for $F_{\mathrm{II}}$ has already its final form

$$
\left\|F_{\mathrm{II}}\right\|_{2}+\left\|\left(1+x^{2}\right) F_{\mathrm{II}}\right\|_{1}=\mathcal{R}_{2}\left(\omega,|z|+\|f\|_{\infty}\right)(1+t)\left(|z|+\|f\|_{\infty}\right)^{2 r-1} .
$$

For $H_{\mathrm{I}}$, we have

$$
\begin{aligned}
\left\|\left(1+x^{2}\right) H_{\mathrm{I}}\right\|_{2}= & \mathcal{R}_{2}\left(\omega,|z|+\|f\|_{\infty}\right)\left[|z|^{3}+\left(|z|+\|f\|_{\infty}\right)\|f\|_{\rho}\right. \\
& \left.+\left(\left|\omega_{T}-\omega\right|+\left(|z|+\|f\|_{\rho}\right)^{2}\right)\left(|z|^{2}+\|f\|_{\rho}\right)\right] \\
= & \mathcal{R}_{2}\left(\omega,|z|+\|f\|_{\infty}\right)\left(|z|^{2}+\|f\|_{\rho}\right) \\
& \times\left[|z|+\|f\|_{\infty}+\left|\omega_{T}-\omega\right|+\left(|z|+\|f\|_{\rho}\right)^{2}\right] \\
= & \mathcal{R}_{2}\left(\omega,|z|+\|f\|_{\infty}\right)\left(|z|^{2}+\|f\|_{\rho}\right)\left(|z|+\|f\|_{\infty}+\left|\omega_{T}-\omega\right|\right) \\
= & \mathcal{R}_{2}\left(\omega,|z|+\|f\|_{\infty}\right)\left(\left\|k_{1}\right\|_{\rho}+|z|^{2}+\left\|h_{1}\right\|_{\rho}\right) \\
& \times\left(|z|+\|f\|_{\infty}+\left|\omega_{T}-\omega\right|\right) .
\end{aligned}
$$

The last remainder to estimate is $\widehat{H}_{R}=F_{\mathrm{II}}+H_{\mathrm{I}}+H_{\mathrm{II}}$. We have

$$
H_{\mathrm{II}}=\sum_{m, n}\left(C_{T}-2 i \mu_{T} m-0\right)^{-1} P_{T}^{n} \mathcal{A}_{m n}
$$

and

$$
\begin{aligned}
\left\|\mathcal{A}_{m n}\right\|_{2}+\left\|\left(1+x^{2}\right)^{3 / 2} \mathcal{A}_{m n}\right\|_{1}= & \mathcal{R}_{2}\left(\omega,|z|+\|f\|_{\infty}\right)|z|\left[|z|\left|\omega_{T}-\omega\right|+\left(|z|+\|f\|_{\rho}\right)^{2}\right] \\
= & \mathcal{R}_{2}\left(\omega,|z|+\|f\|_{\infty}\right)|z|\left[|z|\left|\omega_{T}-\omega\right|\right. \\
& \left.+\left(\left\|k_{1}\right\|_{\rho}+|z|+\left\|h_{1}\right\|_{\rho}\right)^{2}\right] .
\end{aligned}
$$

\subsubsection{Initial conditions}

We suppose that at $t=0$,

$$
\begin{aligned}
& z(0)=z_{0}, \quad\left|z_{0}\right|=\varepsilon^{1 / 2}, \\
& f(x, 0)=f_{0}(x), \quad N \equiv\left\|f_{0}\right\|_{H^{1}}+\left\|\left(1+x^{2}\right) f_{0}\right\|_{2} \leqslant c \varepsilon^{3 / 2} .
\end{aligned}
$$

Since $\left|z_{1}\right|^{2} \leqslant|z|^{2}+\mathcal{R}(\omega, z)|z|^{3}$, we have also, denoting $z_{10}=z_{1}(0)$,

$$
y_{0}=\left|z_{10}\right|^{2} \leqslant \varepsilon+\mathcal{R}\left(\omega,\left|z_{0}\right|\right) \varepsilon^{3 / 2} .
$$


From the formula $h=P_{T} f=f+\left(P_{d}-P_{T}^{d}\right) f$, we see that

$$
\begin{aligned}
& \left\|h_{0}\right\|_{H^{1}} \leqslant\left\|f_{0}\right\|_{H^{1}}+\mathcal{R}_{1}(\omega)\left|\omega_{T}-\omega\right|\left\|f_{0}\right\|_{\rho}, \\
& \left\|\left(1+x^{2}\right) h_{0}\right\|_{2} \leqslant\left\|\left(1+x^{2}\right) f_{0}\right\|_{2}+\mathcal{R}_{1}(\omega)\left|\omega_{T}-\omega\right|\left\|f_{0}\right\|_{\rho} .
\end{aligned}
$$

Therefore,

$$
\left\|h_{0}\right\|_{H^{1}}+\left\|\left(1+x^{2}\right) h_{0}\right\|_{2} \leqslant c \varepsilon^{3 / 2}+\mathcal{R}_{1}(\omega)\left|\omega_{T}-\omega\right|\left\|f_{0}\right\|_{\rho} .
$$

Finally, at $t=0, h_{1}(x, 0)=h_{10}(x)=h_{0}(x)$.

\section{Majorants}

\subsection{Majorants and remainders}

\subsubsection{Definition}

We define the quantities

$$
\begin{aligned}
& \mathbb{M}_{0}(T)=\sup _{0 \leqslant t \leqslant T}\left|\omega_{T}-\omega(t)\right|\left(\frac{\varepsilon}{1+\varepsilon t}\right)^{-1}, \\
& \mathbb{M}_{1}(T)=\sup _{0 \leqslant t \leqslant T}|z(t)|\left(\frac{\varepsilon}{1+\varepsilon t}\right)^{-1 / 2}, \\
& \mathbb{M}_{2}(T)=\sup _{0 \leqslant t \leqslant T}\|f(t)\|_{\infty}\left(\frac{\varepsilon}{1+\varepsilon t}\right)^{-1 / 2} \log ^{-1}(2+\varepsilon t), \\
& \mathbb{M}_{3}(T)=\sup _{0 \leqslant t \leqslant T}\left\|h_{1}(t)\right\|_{\rho}\left(\frac{\varepsilon}{1+\varepsilon t}\right)^{-3 / 2} \log ^{-1}(2+\varepsilon t),
\end{aligned}
$$

referred to as 'majorants', and denote $\mathbb{M}$ the 4-dimensional vector $\left(\mathbb{M}_{0}, \ldots, \mathbb{M}_{3}\right)$. The goal of this section is to prove that if $\varepsilon$ is sufficiently small, all these quantities are bounded uniformly in $T$.

\subsubsection{Estimates of remainders in terms of majorants}

PROPOSITION 5.1. - The majorant $\mathbb{M}_{0}(T)$ satisfies

$$
\mathbb{M}_{0}(T)=\mathcal{R}\left(\varepsilon^{1 / 2} \mathbb{M}\right)\left[\left(1+\mathbb{M}_{1}^{2}\right)^{2}+\varepsilon^{1 / 2}\left(1+\mathbb{M}^{2}\right)^{3 / 2}\right] .
$$

Proof. - To estimate $\left|\omega_{T}-\omega(t)\right|$, we need first to evaluate $|z|+\|f\|_{\rho}$ and $|z|^{2}+\|f\|_{\rho}$ (see (4.5.1). Starting with estimates (4.5.7)-(4.5.8), we obtain:

$$
\begin{aligned}
|z|+\|f\|_{\rho} & =\mathcal{R}_{2}\left(\omega,|z|+\|f\|_{\infty}\right)\left(\left\|k_{1}\right\|_{\rho}+|z|+\left\|h_{1}\right\|_{\rho}\right) \\
& =\mathcal{R}\left(\varepsilon^{1 / 2} \mathbb{M}\right)\left(\frac{\varepsilon}{(1+t)^{3 / 2}}+\left(\frac{\varepsilon}{1+\varepsilon t}\right)^{1 / 2} \mathbb{M}_{1}+\left(\frac{\varepsilon}{1+\varepsilon t}\right)^{3 / 2} \log (2+\varepsilon t) \mathbb{M}_{3}\right) \\
& =\mathcal{R}\left(\varepsilon^{1 / 2} \mathbb{M}\right)\left(\frac{\varepsilon}{1+\varepsilon t}\right)^{1 / 2}\left(\mathbb{M}_{1}+\varepsilon^{1 / 2}\left(1+\mathbb{M}_{3}\right)\right),
\end{aligned}
$$


and similarly

$$
|z|^{2}+\|f\|_{\rho}=\mathcal{R}\left(\varepsilon^{1 / 2} \mathbb{M}\right) \frac{\varepsilon}{1+\varepsilon t}\left(1+\mathbb{M}_{1}^{2}+\varepsilon^{1 / 2} \mathbb{M}_{3}\right) .
$$

Therefore

$$
\begin{aligned}
\left|\omega_{T}-\omega(t)\right| & =\mathcal{R}\left(\varepsilon^{1 / 2} \mathbb{M}\right) \frac{\varepsilon}{1+\varepsilon t}\left[\left(1+\mathbb{M}_{1}^{2}+\varepsilon^{1 / 2} \mathbb{M}_{3}\right)^{2}+\left(\mathbb{M}_{1}+\varepsilon^{1 / 2}\left(1+\mathbb{M}_{3}\right)\right)^{2}\right] \\
& =\mathcal{R}\left(\varepsilon^{1 / 2} \mathbb{M}\right) \frac{\varepsilon}{1+\varepsilon t}\left[\left(1+\mathbb{M}_{1}^{2}\right)^{2}+\varepsilon^{1 / 2}\left(1+\mathbb{M}^{2}\right)^{3 / 2}\right]
\end{aligned}
$$

PROPOSITION 5.2. - The remainder $Y_{R}$ defined in (4.3.28) satisfies the estimate

$$
\left|Y_{R}\right|=\mathcal{R}\left(\varepsilon^{1 / 2} \mathbb{M}\right) \frac{\varepsilon^{5 / 2}}{(1+\varepsilon t)^{2} \sqrt{\varepsilon t}} \log (2+\varepsilon t)\left(1+\mathbb{M}^{2}\right)^{5 / 2} .
$$

Proof. - The remainder $Y_{R}$ is bounded as follows:

$$
\begin{aligned}
\left|Y_{R}\right|= & \mathcal{R}\left(\varepsilon^{1 / 2} \mathbb{M}\right)\left(\frac{\varepsilon}{1+\varepsilon t}\right)^{1 / 2} \mathbb{M}_{1}\left[\left(\frac{\varepsilon}{(1+t)^{3 / 2}}+\frac{\varepsilon}{1+\varepsilon t} \mathbb{M}_{1}^{2}\right.\right. \\
& \left.+\left(\frac{\varepsilon}{1+\varepsilon t}\right)^{3 / 2} \log (2+\varepsilon t) \mathbb{M}_{3}\right)\left(\frac{\varepsilon}{(1+t)^{3 / 2}}+\frac{\varepsilon}{1+\varepsilon t} \mathbb{M}_{1}^{2}\right. \\
& \left.+\left(\frac{\varepsilon}{1+\varepsilon t}\right)^{3 / 2} \log (2+\varepsilon t) \mathbb{M}_{3}+\left(\frac{\varepsilon}{1+\varepsilon t}\right)^{3 / 2} \mathbb{M}_{0} \mathbb{M}_{1}\right) \\
& \left.+\left(\frac{\varepsilon}{1+\varepsilon t}\right)^{1 / 2} \mathbb{M}_{1}\left(\frac{\varepsilon}{(1+t)^{3 / 2}}+\left(\frac{\varepsilon}{1+\varepsilon t}\right)^{3 / 2} \log (2+\varepsilon t) \mathbb{M}_{3}\right)\right] \\
= & \mathcal{R}\left(\varepsilon^{1 / 2} \mathbb{M}\right)\left(\frac{\varepsilon}{1+\varepsilon t}\right)^{2} \mathbb{M}_{1}\left[\left(\frac{\varepsilon}{1+\varepsilon t}\right)^{1 / 2}\left(1+\mathbb{M}_{1}^{2}+\varepsilon^{1 / 2} \mathbb{M}_{3}\right)\right. \\
& \times\left(1+\mathbb{M}_{1}^{2}+\varepsilon^{1 / 2}\left(\mathbb{M}_{3}+\mathbb{M}_{0} \mathbb{M}_{1}\right)\right) \\
& \left.+\left(\frac{1}{\sqrt{1+t}}+\left(\frac{\varepsilon}{1+\varepsilon t}\right)^{1 / 2} \log (2+\varepsilon t) \mathbb{M}_{3}\right) \mathbb{M}_{1}\right] \\
= & \mathcal{R}\left(\varepsilon ^ { 1 / 2 } \mathbb { M } ^ { 2 } \mathbb { M } _ { 1 } \frac { \varepsilon ^ { 5 / 2 } \operatorname { l o g } ( 2 + \varepsilon t ) } { ( 1 + \varepsilon t ) ^ { 2 } \sqrt { \varepsilon t } } \left[\mathbb{M}_{1}\left(1+\mathbb{M}_{3}\right)\right.\right. \\
& \left.+\left(1+\mathbb{M}_{1}^{2}+\varepsilon^{1 / 2} \mathbb{M}_{3}\right)\left(1+\mathbb{M}_{1}^{2}+\varepsilon^{1 / 2}\left(\mathbb{M}_{3}+\mathbb{M}_{0} \mathbb{M}_{1}\right)\right)\right] \\
= & \mathcal{R}\left(\varepsilon^{1 / 2} \mathbb{M}\right) \frac{\varepsilon^{5 / 2}}{(1+\varepsilon t)^{2} \sqrt{\varepsilon t}} \log (2+\varepsilon t)\left(1+\mathbb{M}^{2}\right)^{5 / 2} .
\end{aligned}
$$

Let us turn now to remainders $H_{R}=F_{\mathrm{II}}+H_{\mathrm{I}}$ and $\widehat{H_{R}}=F_{\mathrm{II}}+H_{\mathrm{I}}+H_{\mathrm{II}}$ in Eqs. (4.4.10) and (4.4.11) for $h$ and $h_{1}$ respectively. We combine these results in

PROPOSITION 5.3. - The remainders $F_{\mathrm{II}}$ and $H_{\mathrm{I}}$ satisfy the upper bounds

$$
\left\|F_{\mathrm{II}}\right\|_{2}+\left\|\left(1+x^{2}\right) F_{\mathrm{II}}\right\|_{1}=\mathcal{R}\left(\varepsilon^{1 / 2} \mathbb{M}\right)\left(\frac{\varepsilon}{1+\varepsilon t}\right)^{3 / 2}\left(\mathbb{M}_{1}^{2}+\mathbb{M}_{2}^{2}\right)^{3}
$$


and

$$
\begin{aligned}
\left\|\left(1+x^{2}\right) H_{\mathrm{I}}\right\|_{2}= & \mathcal{R}\left(\varepsilon^{1 / 2} \mathbb{M}\right)\left(\frac{\varepsilon}{1+\varepsilon t}\right)^{3 / 2} \log (2+\varepsilon t) \\
& \times\left(\left(1+\mathbb{M}_{1}^{2}\right)\left(\mathbb{M}_{1}+\mathbb{M}_{2}\right)+\varepsilon^{1 / 2}\left(1+\mathbb{M}^{2}\right)^{3 / 2}\right) .
\end{aligned}
$$

Proof. - It follows from (3.3.23) that

$$
\begin{aligned}
\left\|F_{\mathrm{II}}\right\|_{2}+\left\|\left(1+x^{2}\right) F_{\mathrm{II}}\right\|_{1} & =\mathcal{R}_{2}\left(\omega,|z|+\|f\|_{\infty}\right)(1+t)\left(|z|+\|f\|_{\infty}\right)^{2 r-1} \\
& =\mathcal{R}\left(\varepsilon^{1 / 2} \mathbb{M}\right)\left(\frac{\varepsilon}{1+\varepsilon t}\right)^{3 / 2}\left(\mathbb{M}_{1}^{2}+\mathbb{M}_{2}^{2}\right)^{3} .
\end{aligned}
$$

For $H_{\mathrm{I}}$, see (4.2.19), we have

$$
\begin{aligned}
\left\|\left(1+x^{2}\right) H_{\mathrm{I}}\right\|_{2}= & \mathcal{R}_{2}\left(\left\|k_{1}\right\|_{\rho}+|z|^{2}+\left\|h_{1}\right\|_{\rho}\right)\left(|z|+\|f\|_{\infty}+\left|\omega_{T}-\omega\right|\right) \\
= & \mathcal{R}\left(\varepsilon^{1 / 2} \mathbb{M}\right)\left(\frac{\varepsilon}{(1+t)^{3 / 2}}+\frac{\varepsilon}{1+\varepsilon t} \mathbb{M}_{1}^{2}+\left(\frac{\varepsilon}{1+\varepsilon t}\right)^{3 / 2} \log (2+\varepsilon t) \mathbb{M}_{3}\right) \\
& \times\left(\left(\frac{\varepsilon}{1+\varepsilon t}\right)^{1 / 2} \mathbb{M}_{1}+\left(\frac{\varepsilon}{1+\varepsilon t}\right)^{1 / 2} \log (2+\varepsilon t) \mathbb{M}_{2}+\frac{\varepsilon}{1+\varepsilon} \mathbb{M}_{0}\right) \\
= & \mathcal{R}\left(\varepsilon^{1 / 2} \mathbb{M}\right)\left(\frac{\varepsilon}{1+\varepsilon t}\right)^{3 / 2} \log (2+\varepsilon t) \\
& \times\left(\left(1+\mathbb{M}_{1}^{2}\right)\left(\mathbb{M}_{1}+\mathbb{M}_{2}\right)+\varepsilon^{1 / 2}\left(1+\mathbb{M}^{2}\right)^{3 / 2}\right) .
\end{aligned}
$$

The right-hand side of Eq. (4.4.10) for $h$ contains also $-P_{T} j E_{2}[w, w]$ whose bound can be given as follows

PROPOSITION 5.4.-

$$
\left\|\left(1+x^{2}\right) P_{T} j E_{2}[w, w]\right\|_{2}=\mathcal{R}\left(\varepsilon^{1 / 2} \mathbb{M}\right) \frac{\varepsilon}{1+\varepsilon t} \mathbb{M}_{1}^{2} .
$$

The last remainder to examine is $\widehat{H}_{R}=F_{\mathrm{II}}+H_{\mathrm{I}}+H_{\mathrm{II}}$. We have already estimated $F_{\text {II }}$ and $H_{\mathrm{I}}$ and need only to consider $H_{\mathrm{II}}$ which is given represented in (4.5.16).

PROPOSITION 5.5.-

$$
\left\|\mathcal{A}_{m n}\right\|_{2}+\left\|\left(1+x^{2}\right)^{3 / 2} \mathcal{A}_{m n}\right\|_{1}=\mathcal{R}\left(\varepsilon^{1 / 2} \mathbb{M}\right)\left(\frac{\varepsilon}{1+\varepsilon t}\right)^{3 / 2} \mathbb{M}_{1}\left(\mathbb{M}_{1}^{2}+\varepsilon^{1 / 2}\left(1+\mathbb{M}^{2}\right)\right) .
$$

Proof. - Estimate (4.5.17) shows that

$$
\begin{aligned}
&\left\|\mathcal{A}_{m n}\right\|_{2}+\left\|\left(1+x^{2}\right)^{3 / 2} \mathcal{A}_{m n}\right\|_{1} \\
&= \mathcal{R}_{2}|z|\left[|z|\left|\omega_{T}-\omega\right|+\left(\left\|k_{1}\right\|_{\rho}+|z|+\left\|h_{1}\right\|_{\rho}\right)^{2}\right] \\
&= \mathcal{R}\left(\varepsilon^{1 / 2} \mathbb{M}\right)\left(\frac{\varepsilon}{1+\varepsilon t}\right)^{1 / 2} \mathbb{M}_{1}\left[\left(\frac{\varepsilon}{1+\varepsilon t}\right)^{3 / 2} \mathbb{M}_{0} \mathbb{M}_{1}\right. \\
&\left.+\left(\frac{\varepsilon}{(1+t)^{3 / 2}}+\left(\frac{\varepsilon}{1+\varepsilon t}\right)^{1 / 2} \mathbb{M}_{1}+\left(\frac{\varepsilon}{1+\varepsilon t}\right)^{3 / 2} \log (2+\varepsilon t) \mathbb{M}_{3}\right)^{2}\right]
\end{aligned}
$$




$$
=\mathcal{R}\left(\varepsilon^{1 / 2} \mathbb{M}\right)\left(\frac{\varepsilon}{1+\varepsilon t}\right)^{3 / 2} \mathbb{M}_{1}\left(\mathbb{M}_{1}^{2}+\varepsilon^{1 / 2}\left(1+\mathbb{M}^{2}\right)\right) .
$$

We have completed the estimations of the remainders in terms of majorants. We now write similar estimates for initial data. Coming back to formulas of Section 4.5.3, we have

$$
\begin{aligned}
& y_{0}=y_{\mid t=0}=\varepsilon+\mathcal{R}\left(\varepsilon^{1 / 2} \mathbb{M}\right) \varepsilon^{3 / 2}=\varepsilon\left(1+\mathcal{R}\left(\varepsilon^{1 / 2} \mathbb{M}\right) \varepsilon^{1 / 2}\right) \\
& \left\|h_{0}\right\|_{H^{1}}+\left\|\left(1+x^{2}\right) h_{0}\right\|_{2} \leqslant N+\mathcal{R}_{1}(\omega)\left|\omega_{T}-\omega\right|\left\|f_{0}\right\|_{\rho} \leqslant c_{0} \varepsilon^{3 / 2}+\mathcal{R}\left(\varepsilon^{1 / 2} \mathbb{M}\right) \varepsilon^{2} \mathbb{M}_{0} \\
& \times\left(1+\mathbb{M}_{1}^{2}+\varepsilon^{1 / 2} \mathbb{M}_{3}\right) .
\end{aligned}
$$

\subsection{Estimates for solutions of the canonical system}

\subsubsection{Canonical equations}

This section is devoted to the study the system

$$
\begin{aligned}
& \dot{\ell}=2 \operatorname{Re}\left(i K_{T}\right) \ell^{2}+\mathcal{L}(t), \\
& \dot{\alpha}=C_{M} \alpha+A(x, t), \\
& \dot{\beta}=C_{M} \beta+B(x, t),
\end{aligned}
$$

where

$$
C_{M}=C_{T}+i \sigma(t)\left(P_{T}^{+}-P_{T}^{-}\right),
$$

and under some assumptions on initial data and the source terms. We assume that $\operatorname{Re}\left(i K_{T}\right)<0$ is a given constant. The operator $C_{T}=C\left(\omega_{T}\right)$ does not depend on $t$ and the structure of its spectrum is known. The function $\sigma$ is a smooth, real-valued function of $t$.

We suppose that the initial conditions satisfy

$$
\begin{aligned}
& \ell(0)=\varepsilon\left|\ell_{0}\right|>0, \\
& \|\alpha(0)\|_{H^{1}}+\left\|\left(1+x^{2}\right) \alpha(0)\right\|_{2} \leqslant \varepsilon^{1 / 2}\left|\alpha_{0}\right|, \\
& \|\beta(0)\|_{H^{1}}+\left\|\left(1+x^{2}\right) \beta(0)\right\|_{2} \leqslant \varepsilon^{3 / 2}\left|\beta_{0}\right| .
\end{aligned}
$$

As for the source terms, we assume that

$$
|\mathcal{L}(t)| \leqslant|\mathcal{L}| \frac{\varepsilon^{5 / 2}}{(1+\varepsilon t)^{2} \sqrt{\varepsilon t}} \log (2+\varepsilon t)
$$

and that $A=A_{1}+A_{2}$ with the following hypothesis:

$$
\begin{aligned}
& \left\|\left(1+x^{2}\right) A_{1}\right\|_{2} \leqslant\left|A_{1}^{\prime}\right| \frac{\varepsilon}{1+\varepsilon t}+\left|A_{1}^{\prime \prime}\right|\left(\frac{\varepsilon}{1+\varepsilon t}\right)^{3 / 2} \log (2+\varepsilon t), \\
& \left\|A_{2}\right\|_{2}+\left\|\left(1+x^{2}\right) A_{2}\right\|_{1} \leqslant\left|A_{2}\right|\left(\frac{\varepsilon}{1+\varepsilon t}\right)^{3 / 2}
\end{aligned}
$$

and $B=B_{1}+B_{2}+B_{3}$ satisfies 


$$
\begin{aligned}
& \left\|\left(1+x^{2}\right) B_{1}\right\|_{2} \leqslant\left|B_{1}\right|\left(\frac{\varepsilon}{1+\varepsilon t}\right)^{3 / 2} \log (2+\varepsilon t), \\
& \left\|B_{2}\right\|_{2}+\left\|\left(1+x^{2}\right) B_{2}\right\|_{1} \leqslant\left|B_{2}\right|\left(\frac{\varepsilon}{1+\varepsilon t}\right)^{3 / 2}, \\
& B_{3}=\sum_{k l}\left(C_{T}-2 i \mu_{T} k-0\right)^{-1} P_{T}^{l} B_{k l} \quad \text { with } \\
& \left\|B_{k l}\right\|_{2}+\left\|\left(1+x^{2}\right) B_{k l}\right\|_{1} \leqslant\left|B_{k l}\right|\left(\frac{\varepsilon}{1+\varepsilon t}\right)^{3 / 2},
\end{aligned}
$$

where all the quantities |.| are supposed to be given constants. All these assumptions are motivated by the estimates of the remainders (see Section 5.1) and by the final estimates we intend to prove on $\omega, z, f$ and $h_{1}$. The evolution equation (5.2.1) for $\ell$ corresponds to Eq. (4.4.6) for $y$ and the assumption (5.2.8) on the source term has the form of estimate (5.1.9) for the remainder $Y_{R}$. Similarly, (5.2.2) corresponds to Eq. (4.4.10) for $h$ and assumptions (5.2.9)-(5.2.10) on the source term $A$ have the form of estimates (5.1.11)-(5.1.14) for the remainder $H_{R}=F_{\mathrm{II}}+H_{\mathrm{I}}-P_{T} j E_{2}[w, w]$. Finally, Eq. (5.2.3) corresponds to Eq. (4.4.11) for $h_{1}$ and assumptions (5.2.11)-(5.2.14) to inequality (5.1.15).

\subsubsection{A Ricatti equation}

Eq. (5.2.1) for $\ell$ is of Ricatti type. Introducing the new variable $s=2 \operatorname{Im} K_{T} t$, and denoting $q(s)=\frac{1}{2 \operatorname{Im} K_{T}} \mathcal{L}(t)$, it becomes

$$
\frac{d \ell}{d s}=-\ell^{2}+q(s)
$$

with the assumption on $q$ that

$$
|q(s)| \leqslant|\mathcal{L}| \frac{1}{2 \operatorname{Im} K_{T}} \frac{\varepsilon^{5 / 2}}{(1+\varepsilon t)^{2}} \frac{1}{\sqrt{\varepsilon t}} \log (2+\varepsilon t) .
$$

PROPOSITION 5.6. - The solution of (5.2.1) with an initial condition and a source term satisfying (5.2.5) and (5.2.8) respectively is bounded as follows:

$$
\left|\ell-\frac{\varepsilon\left|\ell_{0}\right|}{1+\varepsilon\left|\ell_{0}\right| s}\right| \leqslant \frac{\beta_{3}\left|q_{1}\right|}{1-\varepsilon^{3 / 2}\|\Lambda\|}\left(\frac{\varepsilon}{1+\varepsilon\left|\ell_{0}\right| s}\right)^{3 / 2} \log \left(2+\varepsilon\left|\ell_{0}\right| s\right) .
$$

Proof. - Define a new function $u$ by $\ell=u^{\prime} / u$, with $u^{\prime}=\frac{d u}{d s}$. The equation for $u$ is

$$
u^{\prime \prime}=q u \text {. }
$$

Assuming that $\left.u\right|_{s=0}=1$, the initial condition on $\ell$ implies that $\left.u^{\prime}\right|_{s=0}=\varepsilon\left|\ell_{0}\right|$. The function $u$ satisfies the integral equation

$$
u(s)=1+\varepsilon\left|\ell_{0}\right| s+\int_{0}^{s}\left(s-s_{1}\right) q\left(s_{1}\right) u\left(s_{1}\right) d s_{1} .
$$


It is convenient to introduce $\sigma=1+\varepsilon\left|\ell_{0}\right| s$. Then,

$$
u(\sigma)=\sigma+\varepsilon^{1 / 2} \int_{1}^{\sigma}\left(\sigma-\sigma_{1}\right) \tilde{q}\left(\sigma_{1}\right) u\left(\sigma_{1}\right) d \sigma_{1}
$$

where

$$
|\tilde{q}(\sigma)| \leqslant\left|q_{0}\right| \frac{1}{\sigma^{2} \sqrt{\sigma-1}} \log \left(2+\frac{\sigma-1}{m}\right),
$$

where $m=\min \left\{1,\left(2\left|\ell_{0}\right| \operatorname{Im} K_{T}\right)^{2}\right\} \leqslant 1$ and

$$
\left|q_{0}\right| \leqslant|\mathcal{L}| \frac{\left|\ell_{0}\right|^{1 / 2}\left(2 \operatorname{Im} K_{T}\right)^{3 / 2}}{m} .
$$

We now rewrite the integral equation (5.2.20) in the form

$$
u=u_{0}+\varepsilon^{1 / 2} \Lambda u, \quad u_{0}=\sigma .
$$

Consider the space of continuous functions on $\sigma \in[1, \infty)$ equipped with the norm

$$
\|u\|=\max \frac{|u(\sigma)|}{\sigma} .
$$

In this space, $\Lambda$ is a bounded operator, whose norm is bounded by

$$
\|\Lambda\| \leqslant \beta_{0}\left|q_{1}\right|
$$

where $\left|q_{1}\right|=\left|q_{0}\right| \log \left(1+\frac{1}{m}\right)$, and $\beta_{0}$ is a constant. Therefore, for sufficiently small $\varepsilon$, there exists a solution $u$ such that

$$
|u| \leqslant \frac{\sigma}{1-\varepsilon^{1 / 2}\|\Lambda\|} .
$$

To get an estimate on $\ell$, we need both an estimate on $u$ and $u^{\prime}$. For this purpose, we write (5.2.20) in the form

$$
u=D \sigma+\varepsilon^{1 / 2} v
$$

where

$$
\begin{aligned}
& D=1+\varepsilon^{1 / 2} \int_{1}^{\infty} d \sigma_{1} \tilde{q}\left(\sigma_{1}\right) u\left(\sigma_{1}\right)=1+\varepsilon^{1 / 2} D_{1}, \\
& v=-\sigma \int_{\sigma}^{\infty} d \sigma_{1} \tilde{q}\left(\sigma_{1}\right) u\left(\sigma_{1}\right)-\int_{1}^{\sigma} d \sigma_{1} \sigma_{1} \tilde{q}\left(\sigma_{1}\right) u\left(\sigma_{1}\right) .
\end{aligned}
$$

It is clear that

$$
v^{\prime}=-\int_{\sigma}^{\infty} d \sigma_{1} \tilde{q}\left(\sigma_{1}\right) u\left(\sigma_{1}\right)
$$


Notice that

$$
\left|D_{1}\right| \leqslant \frac{\beta_{2}\left|q_{1}\right|}{1-\varepsilon^{1 / 2}\|\Lambda\|}
$$

We have

$$
\begin{aligned}
& \left|v^{\prime}\right| \leqslant \frac{\beta_{2}\left|q_{1}\right|}{1-\varepsilon^{1 / 2}\|\Lambda\|} \sigma^{-1 / 2} \log (1+\sigma), \\
& |v| \leqslant \frac{\beta_{2}\left|q_{1}\right|}{1-\varepsilon^{1 / 2}\|\Lambda\|} \sigma^{1 / 2} \log (1+\sigma),
\end{aligned}
$$

where $\beta_{2}$ is a constant. Coming back to $\ell$, we have

$$
\ell=\frac{u_{s}}{u}=\varepsilon \ell_{0} \frac{u_{\sigma}}{u}=\frac{\varepsilon\left|\ell_{0}\right|}{\sigma}\left(1+\frac{\varepsilon^{1 / 2}\left(v_{\sigma}-v / \sigma\right)}{D+\varepsilon^{1 / 2} v / \sigma}\right) .
$$

It follows that $\ell$ satisfies the estimate

$$
\left|\ell-\frac{\varepsilon\left|\ell_{0}\right|}{\sigma}\right| \leqslant \frac{\varepsilon^{3 / 2}}{\sigma^{3 / 2}} \frac{\beta_{3}\left|q_{1}\right|}{1-\varepsilon^{3 / 2}\|\Lambda\|} \log (1+\sigma) .
$$

\subsubsection{Eqs. (5.2.6)-(5.2.7) for $\alpha$ and $\beta$}

The projections $P_{ \pm}(\omega)$ are bounded operators in $L^{2}$, uniformly in $\omega$ for $\omega$ in a compact interval where Assumptions (SP) and (SL) hold.

Define the weighted norms

$$
\|h\|_{W_{1}}=\left\|\left(1+x^{2}\right) h\right\|_{1}, \quad \text { and } \quad\|h\|_{W_{2}}=\left\|\left(1+x^{2}\right) h\right\|_{2} .
$$

LEMMA 5.1.-

$$
\begin{gathered}
\left\|\mathrm{e}^{C_{T} t} P_{T}^{ \pm} \alpha\right\|_{2} \leqslant c\left(\omega_{T}\right)\|\alpha\|_{2} \\
\left\|\mathrm{e}^{C_{T} t} P_{T}^{ \pm} \alpha\right\|_{\infty} \leqslant c\left(\omega_{T}\right)\left\{\begin{array}{l}
t^{-1 / 2}\left(\|\alpha\|_{2}+\|\alpha\|_{W}\right) \\
(1+t)^{-1 / 2}\left(\|\alpha\|_{H^{1}}+\|\alpha\|_{W}\right)
\end{array}\right.
\end{gathered}
$$

where $\|h\|_{W}$ stands for either $\|h\|_{W_{1}}$ or $\|h\|_{W_{2}}$, and the constant $c\left(\omega_{T}\right)$ depends on $C_{T}$ and thus on $\omega_{T}$.

We have also

$$
\left\|\mathrm{e}^{C_{T} t} P_{T}^{ \pm} h\right\|_{\rho} \leqslant c\left(\omega_{T}\right)(1+t)^{-3 / 2}\left[\|h\|_{2}+\|h\|_{W}\right],
$$

where $\rho(x)=\left(1+x^{2}\right)^{-q}, q \geqslant 2$.

LEMMA 5.2.-

$$
\begin{gathered}
\left\|\mathrm{e}^{C_{T} t} C_{T}^{-1} P_{T}^{ \pm} h\right\|_{\rho} \leqslant c(1+t)^{-3 / 2}\left\|\left(1+x^{2}\right) h\right\|_{2}, \\
\left\|\mathrm{e}^{C_{T} t}\left(C_{T} \pm 2 i \mu-0\right)^{-1} P_{T}^{ \pm} h\right\|_{\rho} \leqslant c(1+t)^{-3 / 2}\left[\|h\|_{2}+\left\|\left(1+x^{2}\right)^{3 / 2} h\right\|_{1}\right],
\end{gathered}
$$

where $\rho(x)=\left(1+x^{2}\right)^{-q}, q \geqslant 2$. 
Proofs of Lemmas 5.1 and 5.2 can be found in [2]. Some additional comments are given in Appendix A.

LEMMA 5.3. - We have the upper bounds for the following integrals:

$$
\begin{aligned}
& \int_{0}^{t} \frac{d s}{(t-s)^{1 / 2}}\left(\frac{\varepsilon}{1+\varepsilon s}\right)^{\gamma} \leqslant c\left(\frac{\varepsilon}{1+\varepsilon t}\right)^{1 / 2} \varepsilon^{\gamma-1} \text { for } \gamma>1, \\
& \int_{0}^{t} \frac{d s}{(1+t-s)^{3 / 2}}\left(\frac{\varepsilon}{1+\varepsilon s}\right)^{\gamma} \leqslant c\left(\frac{\varepsilon}{1+\varepsilon t}\right)^{3 / 2} \varepsilon^{\gamma-3 / 2} \text { for } \gamma \geqslant 3 / 2, \\
& \int_{0}^{t} \frac{d s}{(t-s)^{1 / 2}} \frac{\varepsilon}{1+\varepsilon s} \leqslant c\left(\frac{\varepsilon}{1+\varepsilon t}\right)^{1 / 2} \log (2+\varepsilon t), \\
& \int_{0}^{t} \frac{d s}{(1+t-s)^{3 / 2}}\left(\frac{\varepsilon}{1+\varepsilon t}\right)^{3 / 2} \log (2+\varepsilon s) \leqslant c\left(\frac{\varepsilon}{1+\varepsilon t}\right)^{3 / 2} \log (2+\varepsilon t) .
\end{aligned}
$$

We give the details of the proof only for inequalities (5.2.44) and (5.2.43). The other upper bounds are obtained in a similar way.

Let us denote

$$
I=\int_{0}^{t} \frac{1}{(t-s)^{1 / 2}} \frac{\varepsilon}{1+\varepsilon s} d s .
$$

If $\varepsilon t \leqslant 1$, we immediately get

$$
I \leqslant C \varepsilon^{1 / 2},
$$

When $\varepsilon t \gg 1$, we separate $I$ into $I=I_{1}+J$, into $I_{1}=\int_{0}^{1 / \varepsilon}$ and $J=\int_{1 / \varepsilon}^{t}$. Then

$$
I_{1} \leqslant \varepsilon\left[t^{1 / 2}-\left(t-\frac{1}{\varepsilon}\right)^{1 / 2}\right] \leqslant C\left(\frac{\varepsilon}{1+\varepsilon t}\right)^{1 / 2}
$$

and

$$
J \leqslant t^{-1 / 2} \int_{1 / \varepsilon t}^{1} \frac{d \sigma}{(1-\sigma)^{1 / 2} \sigma}
$$

leading to

$$
I \leqslant C\left(\frac{\varepsilon}{1+\varepsilon t}\right)^{1 / 2}(1+\log (\varepsilon t)) \leqslant C\left(\frac{\varepsilon}{1+\varepsilon t}\right)^{1 / 2} \log (2+\varepsilon t) .
$$

Denote

$$
I=\int_{0}^{t} \frac{d s}{(1+t-s)^{3 / 2}}\left(\frac{\varepsilon}{1+\varepsilon s}\right)^{\gamma}
$$


If $\varepsilon t \leqslant 1$,

$$
I \leqslant \varepsilon^{\gamma} \int_{0}^{t} \frac{d s}{(1+t-s)^{3 / 2}}=C \varepsilon^{\gamma}\left[1-\frac{1}{\sqrt{1+t}}\right] \leqslant C \varepsilon^{\gamma} .
$$

When $\varepsilon t \gg 1$, we separate again $I$ into $I=I_{1}+J$, with $I_{1}=\int_{0}^{1 / \varepsilon}$ and $J=\int_{1 / \varepsilon}^{t}$

$$
I_{1}=\varepsilon^{\gamma}\left[\frac{1}{\sqrt{1+t}}-\frac{1}{\sqrt{1+t-1 / \varepsilon}}\right] \leqslant C \varepsilon^{\gamma-1} \frac{1}{(1+t)^{3 / 2}} .
$$

On the other hand, we rewrite $J$ in the form $(s=t \sigma)$

$$
\begin{aligned}
J & =\int_{1 / \varepsilon t}^{1} \frac{t^{1-\gamma} d \sigma}{(1+t(1-\sigma))^{3 / 2} \sigma^{\gamma}} \\
& \leqslant C \int_{1 / \varepsilon t}^{1 / 2} \frac{t^{1-\gamma} d \sigma}{(1+t)^{3 / 2} \sigma^{\gamma}}+\int_{1 / 2}^{1} \frac{t^{1-\gamma} d \sigma}{(1+t(1-\sigma))^{3 / 2}} \leqslant C \frac{\varepsilon^{\gamma-1}}{t^{3 / 2}}+C \frac{1}{t^{\gamma}} .
\end{aligned}
$$

Thus,

$$
I \leqslant C \frac{\varepsilon^{\gamma-1}}{t^{3 / 2}}+\frac{1}{t^{\gamma}} \leqslant C \frac{\varepsilon^{\gamma}}{(\varepsilon t)^{3 / 2}} .
$$

Estimate (5.2.43) follows.

Proposition 5.7. - The function $\alpha(x, t)$ solution of (5.2.2) is expressed as

$$
\alpha=\mathrm{e}^{C_{T} t+i \int_{0}^{t} \sigma\left(t_{1}\right) d t_{1}\left(P_{T}^{+}-P_{T}^{-}\right)} \alpha_{0}+\int_{0}^{t} \mathrm{e}^{C_{T}(t-s)+i \int_{s}^{t} \sigma\left(t_{1}\right) d t_{1}\left(P_{T}^{+}-P_{T}^{-}\right)} A(s) d s
$$

and satisfies

$$
\|\alpha\|_{\infty} \leqslant c\left(\omega_{T}\right)\left(\frac{\varepsilon}{1+\varepsilon t}\right)^{1 / 2} \log (2+\varepsilon t)\left(\left|\alpha_{0}\right|+\left|A_{1}^{\prime}\right|+\varepsilon^{1 / 2}\left(\left|A_{1}^{\prime \prime}\right|+\left|A_{2}\right|\right)\right) .
$$

Proof. - In the integral representation (5.2.56), we have first to estimate $\mathrm{e}^{i \int_{s}^{t} \sigma\left(t_{1}\right) d t_{1}\left(P_{T}^{+}-P_{T}^{-}\right)}$. Notice first that, denoting $\nu=\int_{s}^{t} \sigma\left(t_{1}\right) d t_{1}$, we have

$$
\begin{gathered}
\mathrm{e}^{i v P_{+}}=P_{+} \mathrm{e}^{i v}+P_{-}, \\
\mathrm{e}^{i v\left(P_{+}-P_{-}\right)}=\left(P_{+} \mathrm{e}^{i v}+P_{-}\right)\left(P_{-} \mathrm{e}^{-i \nu}+P_{+}\right)=P_{+} \mathrm{e}^{i v}+P_{-} \mathrm{e}^{-i v},
\end{gathered}
$$

and

$$
\mathrm{e}^{\int_{0}^{t} C_{M}\left(t_{1}\right) d t_{1}}=\left(\mathrm{e}^{i v} P_{T}^{+}+\mathrm{e}^{-i v} P_{T}^{-}\right) \mathrm{e}^{C_{T} t} .
$$

Since $\sigma$ is a real function, both exponentials are bounded. Using Lemmas 5.1 and 5.2 on the linear evolution, we get 


$$
\begin{aligned}
\|\alpha\|_{\infty} \leqslant & c\left(\omega_{T}\right)\left[\left|\alpha_{0}\right|\left(\frac{\varepsilon}{1+t}\right)^{1 / 2}+\int_{0}^{t} \frac{1}{(t-s)^{1 / 2}}\left(\left|A_{1}^{\prime}\right| \frac{\varepsilon}{1+\varepsilon s}\right.\right. \\
& \left.\left.+\left|A_{1}^{\prime \prime}\right|\left(\frac{\varepsilon}{1+\varepsilon s}\right)^{3 / 2} \log (2+\varepsilon s)+\left|A_{2}\right|\left(\frac{\varepsilon}{1+\varepsilon s}\right)^{3 / 2}\right) d s\right] .
\end{aligned}
$$

Computing the integrals with the help of Lemma 5.3, we get

$$
\begin{aligned}
\|\alpha\|_{\infty} \leqslant & c\left(\omega_{T}\right)\left[\left|\alpha_{0}\right|\left(\frac{\varepsilon}{1+\varepsilon t}\right)^{1 / 2}+\left|A_{1}^{\prime}\right|\left(\frac{\varepsilon}{1+\varepsilon t}\right)^{1 / 2} \log (2+\varepsilon t)\right. \\
& \left.+\left|A_{1}^{\prime \prime}\right|\left(\frac{\varepsilon}{1+\varepsilon t}\right)^{1 / 2} \varepsilon^{1 / 2}+\left|A_{2}\right|\left(\frac{\varepsilon}{1+\varepsilon t}\right)^{1 / 2} \varepsilon^{1 / 2}\right] .
\end{aligned}
$$

We finally obtain that

$$
\|\alpha\|_{\infty} \leqslant c\left(\omega_{T}\right)\left(\frac{\varepsilon}{1+\varepsilon t}\right)^{1 / 2} \log (2+\varepsilon t)\left[\left|\alpha_{0}\right|+\left|A_{1}^{\prime}\right|+\varepsilon^{1 / 2}\left(\left|A_{1}^{\prime \prime}\right|+\left|A_{2}\right|\right)\right] .
$$

PROPOSITION 5.8. - The function $\beta$ solution of (5.2.3) has the integral representation

$$
\beta=\mathrm{e}^{C_{T} t+i \int_{0}^{t} \sigma\left(t_{1}\right) d t_{1}\left(P_{T}^{+}-P_{T}^{-}\right)} \beta_{0}+\int_{0}^{t} \mathrm{e}^{C_{T}(t-s)+i \int_{s}^{t} \sigma\left(t_{1}\right) d t_{1}\left(P_{T}^{+}-P_{T}^{-}\right)} B(s) d s
$$

and satisfies

$$
\|\beta\|_{\rho} \leqslant c\left(\omega_{T}\right)\left(\frac{\varepsilon}{1+\varepsilon t}\right)^{3 / 2} \log (2+\varepsilon t)\left[\left|\beta_{0}\right|+\left|B_{1}\right|+\left|B_{2}\right|+\sum\left|B_{m n}\right|\right] .
$$

Proof. - From the integral representation, we have

$$
\begin{aligned}
\|\beta\|_{\rho} \leqslant & c\left(\omega_{T}\right)\left[\left|\beta_{0}\right|\left(\frac{\varepsilon}{1+t}\right)^{3 / 2}+\int_{0}^{t} \frac{d s}{(1+s-t)^{3 / 2}}\left(\left|B_{1}\right|\left(\frac{\varepsilon}{1+\varepsilon s}\right)^{3 / 2} \log (2+\varepsilon s)\right.\right. \\
& \left.\left.+\left|B_{2}\right|\left(\frac{\varepsilon}{1+\varepsilon s}\right)^{3 / 2}+\sum\left|B_{m n}\right|\left(\frac{\varepsilon}{1+\varepsilon s}\right)^{3 / 2}\right)\right]
\end{aligned}
$$

As above, we get

$$
\begin{aligned}
\|\beta\|_{\rho} \leqslant & c\left(\omega_{T}\right)\left[\left|\beta_{0}\right|\left(\frac{\varepsilon}{1+t}\right)^{3 / 2}+\left|B_{1}\right|\left(\frac{\varepsilon}{1+t}\right)^{3 / 2} \log (2+\varepsilon t)\right. \\
& \left.+\left(\left|B_{2}\right|+\sum\left|B_{m n}\right|\right)\left(\frac{\varepsilon}{1+\varepsilon t}\right)^{3 / 2}\right]
\end{aligned}
$$

and thus (5.2.65). 


\subsection{Estimates for majorants}

In this section, we establish inequalities for the $\mathbb{M}_{i}$. The equation satisfied by $\omega_{1}$ is

$$
\dot{\omega}_{1}=\widehat{\Omega_{R}}
$$

and the estimate for $\mathbb{M}_{0}$ was obtained in Proposition 5.1

$$
\mathbb{M}_{0}(T) \leqslant \mathcal{R}\left(\varepsilon^{1 / 2} \mathbb{M}\right)\left[\left(1+\mathbb{M}_{1}^{2}\right)^{2}+\varepsilon^{1 / 2}\left(1+\mathbb{M}^{2}\right)^{3 / 2}\right]
$$

The equation satisfied by $y$ is

$$
\dot{y}=\operatorname{Re}\left(i K_{T}\right) y^{2}+Y_{R}
$$

and the remainder $Y_{R}$ satisfies the inequality (5.1.9). which is exactly the condition (5.2.8) assumed on the source term of the model Ricatti equation (5.2.1) with $|\mathcal{L}|=$ $\mathcal{R}\left(\varepsilon^{1 / 2} \mathbb{M}\right)\left(1+\mathbb{M}^{2}\right)^{5 / 2}$. Using (5.2.35) as well as (5.1.17) to bound the initial condition $y_{0}$, it follows that

$$
y \leqslant \mathcal{R}\left(\varepsilon^{1 / 2} \mathbb{M}\right)\left\{\frac{\varepsilon+\varepsilon^{3 / 2}}{1+\varepsilon t}+\left(\frac{\varepsilon}{1+\varepsilon t}\right)^{3 / 2} \log (2+\varepsilon t)\left(1+\mathbb{M}^{2}\right)^{5 / 2}\right\} .
$$

Using that $|z|^{2} \leqslant y+\mathcal{R}(\omega,|z|)|z|^{3}$, we get

$$
\begin{aligned}
|z|^{2} \leqslant & \mathcal{R}\left(\varepsilon^{1 / 2} \mathbb{M}\right)\left\{\frac{\varepsilon+\varepsilon^{3 / 2}}{1+\varepsilon t}+\left(\frac{\varepsilon}{1+\varepsilon t}\right)^{3 / 2} \log (2+\varepsilon t)\left(1+\mathbb{M}^{2}\right)^{5 / 2}\right\} \\
& +\mathcal{R}\left(\varepsilon^{1 / 2} \mathbb{M}\right) \mathbb{M}_{1}^{3} \frac{\varepsilon^{3 / 2}}{(1+\varepsilon t)^{3 / 2}}
\end{aligned}
$$

which yields

Proposition 5.9. - We have

$$
\mathbb{M}_{1}^{2} \leqslant \mathcal{R}\left(\varepsilon^{1 / 2} \mathbb{M}\right)\left(1+\varepsilon^{1 / 2} F_{5}(\mathbb{M})\right)
$$

where $F_{\alpha}(r)=\left(1+r^{2}\right)^{\alpha / 2}$.

We now turn to $\|f\|_{\infty}$. From (4.5.10), we have

$$
\begin{aligned}
\|f\|_{\infty} \leqslant & \|h\|_{\infty}+\mathcal{R}_{2}\left|\omega_{T}-\omega\right|\left(\left\|k_{1}\right\|_{\rho}+|z|^{2}+\left\|h_{1}\right\|_{\rho}\right) \\
\leqslant & \|h\|_{\infty}+\mathcal{R}\left(\varepsilon^{1 / 2} \mathbb{M}\right) \mathbb{M}_{0} \frac{\varepsilon}{1+\varepsilon t}\left[\frac{\varepsilon}{1+t}+\mathbb{M}_{1}^{2} \frac{\varepsilon}{1+\varepsilon t}\right. \\
& \left.\quad+\mathbb{M}_{3}\left(\frac{\varepsilon}{1+\varepsilon t}\right)^{3 / 2} \log (2+\varepsilon t)\right] \\
\leqslant & \|h\|_{\infty}+\varepsilon^{1 / 2} \mathcal{R}\left(\varepsilon^{1 / 2} \mathbb{M}\right)\left(\frac{\varepsilon}{1+\varepsilon t}\right)^{1 / 2} \mathbb{M}_{0}\left(1+\mathbb{M}_{1}^{2}+\varepsilon^{1 / 2} \mathbb{M}_{3}\right)
\end{aligned}
$$

To bound $\|h\|_{\infty}$, we apply the result of Proposition 5.8. Indeed, Eq. (4.4.10) for $h$ has the form (5.2.2) with $A=-P_{T} j E_{2}[w, w]+H_{\mathrm{I}}+F_{\mathrm{II}}$. More precisely, $P_{T} E_{2}[w, w], H_{\mathrm{I}}$, 
and $F_{\text {II }}$ satisfy the hypothesis (5.2.9)-(5.2.10) on $A_{1}^{\prime}, A_{1}^{\prime \prime}$ and $A_{2}$ respectively. Indeed, using (5.1.11)-(5.1.14), we have

$$
\begin{aligned}
& \left|A_{1}^{\prime}\right|=\mathcal{R}\left(\varepsilon^{1 / 2} \mathbb{M}\right) \mathbb{M}_{1}^{2}, \\
& \left|A_{1}^{\prime \prime}\right|=\mathcal{R}\left(\varepsilon^{1 / 2} \mathbb{M}\right)\left(\left(1+\mathbb{M}_{1}^{2}\right)\left(\mathbb{M}_{1}+\mathbb{M}_{2}\right)+\varepsilon^{1 / 2}\left(1+\mathbb{M}^{2}\right)^{3 / 2}\right), \\
& \left|A_{2}\right|=\mathcal{R}\left(\varepsilon^{1 / 2} \mathbb{M}\right)\left(\mathbb{M}_{1}^{2}+\mathbb{M}_{2}^{2}\right)^{3} .
\end{aligned}
$$

As for the initial condition, we have from (5.1.18)

$$
\left|\alpha_{0}\right| \leqslant c_{0} \varepsilon+\mathcal{R}\left(\varepsilon^{1 / 2} \mathbb{M}\right) \varepsilon^{3 / 2} \mathbb{M}_{0}\left(1+\mathbb{M}_{1}^{2}+\varepsilon^{1 / 2}\left(1+\mathbb{M}^{2}\right)^{1 / 2}\right) .
$$

It follows that

$$
\|h\|_{\infty}=\mathcal{R}\left(\varepsilon^{1 / 2} \mathbb{M}\right)\left(\frac{\varepsilon}{1+\varepsilon t}\right)^{1 / 2} \log (2+\varepsilon t)\left[\left(\mathbb{M}_{1}^{2}+\varepsilon^{1 / 2}\right) F_{3}(\mathbb{M})\right]
$$

Proposition 5.10. - The quantity $\mathbb{M}_{2}$ satisfies

$$
\mathbb{M}_{2}=\mathcal{R}\left(\varepsilon^{1 / 2} \mathbb{M}\right)\left(\mathbb{M}_{1}^{2}+\varepsilon^{1 / 2} F_{3}(\mathbb{M})\right) .
$$

Let us consider at last $h_{1}$ solution of (4.4.11). It has the form of (5.2.3) with $B=\widehat{H_{R}}=H_{\mathrm{I}}+F_{\mathrm{II}}+H_{\mathrm{II}}$, where $H_{\mathrm{I}}, F_{\mathrm{II}}$ and $H_{\mathrm{II}}$ identify respectively to $B_{1}, B_{2}, B_{3}$. More precisely, using (5.1.11), (5.1.12) and (5.1.15), we have

$$
\begin{aligned}
& \left|B_{1}\right|=\mathcal{R}\left(\varepsilon^{1 / 2} \mathbb{M}\right)\left(\left(1+\mathbb{M}_{1}^{2}\right)\left(\mathbb{M}_{1}+\mathbb{M}_{2}\right)+\varepsilon^{1 / 2}\left(1+\mathbb{M}^{2}\right)^{3 / 2}\right), \\
& \left|B_{2}\right|=\mathcal{R}\left(\varepsilon^{1 / 2} \mathbb{M}\right)\left(\mathbb{M}_{1}+\mathbb{M}_{2}\right)^{6}, \\
& \left|B_{m n}\right|=\mathcal{R}\left(\varepsilon^{1 / 2} \mathbb{M}\right) \mathbb{M}_{1}\left(\mathbb{M}_{1}^{2}+\varepsilon^{1 / 2}\left(1+\mathbb{M}^{2}\right)\right) .
\end{aligned}
$$

Concerning the initial conditions, we know that $h_{10}=h_{0}$. Thus

$$
\left|\beta_{0}\right|=c_{0}+\mathcal{R}\left(\varepsilon^{1 / 2} \mathbb{M}\right) \varepsilon^{1 / 2} \mathbb{M}_{0}\left(1+\mathbb{M}_{1}^{2}+\varepsilon^{1 / 2}\left(1+\mathbb{M}^{2}\right)^{1 / 2}\right) .
$$

Applying Proposition 5.8, we have

$$
\begin{aligned}
\|h\|_{\rho}= & \mathcal{R}\left(\varepsilon^{1 / 2} \mathbb{M}\right)\left(\frac{\varepsilon}{1+\varepsilon t}\right)^{3 / 2} \log (2+\varepsilon t) \\
& \times\left[\left(1+\mathbb{M}_{1}^{2}\right)\left(\mathbb{M}_{1}+\mathbb{M}_{2}\right)+\left(\mathbb{M}_{1}^{2}+\mathbb{M}_{2}^{2}\right)^{3}+\varepsilon^{1 / 2}\left(1+\mathbb{M}^{2}\right)^{3 / 2}\right] .
\end{aligned}
$$

PROPOSITION 5.11. - The function $h_{1}$ satisfies the estimate

$$
\mathbb{M}_{3}=\mathcal{R}\left(\varepsilon^{1 / 2} \mathbb{M}\right)\left[1+\left(\mathbb{M}_{1}^{2}+\mathbb{M}_{2}^{2}\right)^{3}+\varepsilon^{1 / 2} F_{3}(\mathbb{M})\right]
$$

\subsection{Uniform bounds for $\mathbb{M}$}

The aim of this section is to prove that if $\varepsilon$ is sufficiently small, all the $\mathbb{M}_{i}$ are bounded uniformly in $T$ and $\varepsilon$.

We now have the system (5.1.5),(5.3.6), (5.3.13) and (5.3.19) for the $\mathbb{M}_{i}, i=0,1,2,3$, rewritten in the form 


$$
\begin{aligned}
& \mathbb{M}_{0}=\mathcal{R}\left(\varepsilon^{1 / 2} \mathbb{M}\right)\left[\left(1+\mathbb{M}_{1}^{2}\right)^{2}+\varepsilon^{1 / 2} F_{3}(\mathbb{M})\right] \\
& \mathbb{M}_{1}^{2}=\mathcal{R}\left(\varepsilon^{1 / 2} \mathbb{M}\right)\left[1+\varepsilon^{1 / 2} F_{5}(\mathbb{M})\right] \\
& \mathbb{M}_{2}=\mathcal{R}\left(\varepsilon^{1 / 2} \mathbb{M}\right)\left[\mathbb{M}_{1}^{2}+\varepsilon^{1 / 2} F_{3}(\mathbb{M})\right] \\
& \mathbb{M}_{3}=\mathcal{R}\left(\varepsilon^{1 / 2} \mathbb{M}\right)\left[1+\left(\mathbb{M}_{1}^{2}+\mathbb{M}_{2}^{2}\right)^{3}+\varepsilon^{1 / 2} F_{3}(\mathbb{M})\right] .
\end{aligned}
$$

PROPOSITION 5.12. - For $\varepsilon$ sufficiently small, there exists a constant $M$ independent of $T$ and $\varepsilon$, such that,

$$
|\mathbb{M}(T)| \leqslant M .
$$

Proof. - Combining the inequalities for the $\mathbb{M}_{i}$, one get a estimate of the form

$$
\mathbb{M}^{2} \leqslant \mathcal{R}\left(\varepsilon^{1 / 2} \mathbb{M}\right)\left[\left(1+\mathbb{M}_{1}^{2}+\mathbb{M}_{2}^{2}\right)^{6}+\varepsilon^{1 / 2} F_{3}(\mathbb{M})\right]
$$

Replacing $\mathbb{M}_{2}^{2}$ in the right-hand side of (5.4.6), by its bound (5.4.3) in terms of $\mathbb{M}_{1}^{2}$, and again $\mathbb{M}_{1}$ by its upper bound (5.4.2), we get an inequality in the form

$$
\mathbb{M}^{2} \leqslant \mathcal{R}\left(\varepsilon^{1 / 2} \mathbb{M}\right)\left(1+\varepsilon^{1 / 2} F(\mathbb{M})\right),
$$

where $F(r)$ is a function with finite power growth. From this inequality, it follows, that $\mathbb{M}$ is either bounded independently of $\varepsilon$ or $\mathbb{M}$ belongs to a set separated from the origin $|\mathbb{M}|>k(\varepsilon)$, with $k(\varepsilon) \rightarrow \infty$ as $\varepsilon \rightarrow 0$. Indeed, suppose that $\mathbb{M}(T)$ is such that $\varepsilon^{1 / 2} \mathbb{M}(T) \rightarrow 0$ as $\varepsilon \rightarrow 0$. Then, from inequality (5.4.7), we see that $\mathbb{M}(T) \leqslant K$, independently of $\varepsilon$ and $T$. On the other hand, if $\varepsilon^{1 / 2} \mathbb{M}(T)$ does not tend to 0 as $\varepsilon \rightarrow 0$, then $\mathbb{M}(T)$ is outside a large ball centered at the origin. However, at $t=0, \mathbb{M}(0)$ is bounded independently of $\varepsilon$. This would lead to a discontinuity of $\mathbb{M}$ as a function of time. We conclude that estimate (5.4.5) is true for all $t, t \leqslant T$.

PROPOSITION 5.13. - The function $\omega(t)$ has a limit $\omega_{+}$as $t \rightarrow \infty$. Furthermore, we have the estimates for all $t>0$,

$$
\begin{aligned}
& \left|\omega_{+}-\omega(t)\right| \leqslant \mathbb{M}_{0} \frac{\varepsilon}{1+\varepsilon t}, \\
& |z(t)| \leqslant \mathbb{M}_{1}\left(\frac{\varepsilon}{1+\varepsilon t}\right)^{1 / 2}, \\
& \|f(t)\|_{\infty} \leqslant \mathbb{M}_{2}\left(\frac{\varepsilon}{1+\varepsilon t}\right)^{1 / 2} \log (2+\varepsilon t), \\
& \left\|h_{1}(t)\right\|_{\rho} \leqslant \mathbb{M}_{3}\left(\frac{\varepsilon}{1+\varepsilon t}\right)^{3 / 2} \log (2+\varepsilon t) .
\end{aligned}
$$

In particular, $\left|\omega_{T}-\omega(t)\right| \leqslant \frac{\varepsilon}{1+\varepsilon t}$ and thus $\left|\omega_{T}-\omega(t)\right|$ is a decreasing function of $t$. Applying this result to $\left|\omega\left(t_{1}\right)-\omega\left(t_{2}\right)\right|$, we see that $\omega(t)$ is a Cauchy sequence. It thus has a limit, denoted $\omega_{+}$. We denote by $\mathcal{M}_{i}$ the limiting value $\mathbb{M}_{i}(T)$ as $T \rightarrow \infty$.

Notice that in decomposition (4.4.9) $f=g+h=g+h_{1}+k+k_{1}$, a fixed time $T$ has been chosen, and all the components depend on $\omega(T)$. From the above proposition, we know that $\omega(t)$ has a limit $\omega_{+}$as $t \rightarrow \infty$. So we can reformulate the decomposition by choosing $T=\infty$ and have the dependency of the various components of $f$ on $\omega_{+}$. Let 
us denote $P_{\infty}=P_{c}\left(\omega_{+}\right)$and $P_{\infty}^{d}=I-P_{\infty}$. We define $f=g+h$ where $g=P_{\infty}^{d} f$ and $h=P_{\infty} f$. We also decompose $h=k+k_{1}+h_{1}$ as in (4.4.9)

$$
\begin{aligned}
& k=a_{20} z^{2}+a_{11} z \bar{z}+a_{02} \bar{z}^{2}, \quad k_{0}=k_{\mid t=0}, \\
& k_{1}=-\exp \left(\int_{0}^{t} C_{+}(\tau) d \tau\right) k_{0},
\end{aligned}
$$

where $a_{i j}=a_{i j}\left(\omega_{+}, x\right)$ and $C_{+}=C\left(\omega_{+}\right)+i\left(\omega(t)-\omega_{+}+\dot{\gamma}\right)\left(P_{\infty}^{+}-P_{\infty}^{-}\right)$. All the estimates previously obtained in Sections 4 and 5 under the hypothesis that $T$ is a fixed finite time can be carried out without modification to $T=\infty$ and $\omega_{T}=\omega_{+}$.

We now state the main result of this paper:

THEOREM 1. - Consider the nonlinear Schrödinger equation (1.0.1).

(i) Assume that the nonlinearity satisfies Assumptions (NL) and (SL) and that there exist solutions in the form of solitary waves $\mathrm{e}^{j \omega_{0} t} \phi\left(\omega_{0}\right)$.

(ii) Denoting $B$ the linearized operator near the solitary wave, assume that $C=$ $j^{-1} B$ satisfies the condition (SP) describing the structure of its spectrum.

(iii) Assume the non-degeneracy condition $\left\langle E_{2}[u, u], u\left(2 i \mu_{0}\right)\right\rangle \neq 0$.

(iv) Take an initial condition $\psi_{0}$ in the form of a perturbation of the solitary wave:

$$
\psi_{0}=\phi\left(x, \omega_{0}\right)+\left(z_{0} u\left(x, \omega_{0}\right)+\bar{z}_{0} u^{*}\left(x, \omega_{0}\right)\right)+f_{0}(x)
$$

$$
\text { satisfying }\left|z_{0}\right|=\varepsilon^{1 / 2} \text { and } N \equiv\left\|f_{0}\right\|_{H^{1}}+\left\|\left(1+x^{2}\right) f_{0}\right\|_{2} \leqslant c \varepsilon^{3 / 2} .
$$

For $\varepsilon$ small enough, one can write the solution in the form

$$
\psi(x, t)=\mathrm{e}^{j\left(\int^{t} \omega(s) d s+\gamma(t)\right)}\left(\phi(x, \omega)+z(t) u(x, \omega)+\bar{z}(t) u^{*}(x, \omega)+f(x, t)\right)
$$

with the following properties. There exists a constant $\omega_{+}$such that $\omega_{+}=\lim _{t \rightarrow \infty} \omega(t)$. In addition, for all $t>0$, there exit constants $\mathcal{M}_{0}, \ldots, \mathcal{M}_{3}$ such that

$$
\begin{aligned}
& \left|\omega_{+}-\omega(t)\right| \leqslant \mathcal{M}_{0} \frac{\varepsilon}{1+\varepsilon t}, \\
& |z(t)| \leqslant \mathcal{M}_{1}\left(\frac{\varepsilon}{1+\varepsilon t}\right)^{1 / 2}, \\
& \|f(t)\|_{\infty} \leqslant \mathcal{M}_{2}\left(\frac{\varepsilon}{1+\varepsilon t}\right)^{1 / 2} \log (2+\varepsilon t) .
\end{aligned}
$$

Furthermore, we decompose $f=g+h$, where $g=P_{\infty}^{d} f, h=P_{\infty} f=k+k_{1}+h_{1}$, $k=a_{20} z^{2}+a_{11} z \bar{z}+a_{02} \bar{z}^{2}, k_{0}=k_{\mid t=0}$, and $k_{1}=-\exp \left(\int_{0}^{t} C_{+}(\tau) d \tau\right) k_{0}$. In the above equations, $a_{i j}=a_{i j}\left(\omega_{+}, x\right)$ are defined in (4.2.31) and (4.2.33), and $C_{+}=C\left(\omega_{+}\right)+$ $i\left(\omega_{+}-\omega(t)+\dot{\gamma}\right)\left(P_{\infty}^{+}-P_{\infty}^{-}\right)$.

The function $h_{1}$ satisfies the estimate

$$
\left\|h_{1}(t)\right\|_{\rho} \leqslant \mathcal{M}_{3}\left(\frac{\varepsilon}{1+\varepsilon t}\right)^{3 / 2} \log (2+\varepsilon t) .
$$




\section{Asymptotic behavior of the solution for large time}

\subsection{Long time behavior of $z(t)$ and $\omega(t)$}

We start with Eq. (4.3.20) for $z_{1}$ that we rewrite

$$
\dot{z}_{1}=i \mu z_{1}+i K_{+}\left|z_{1}\right|^{2} z_{1}+\widehat{Z_{R}}
$$

with $K_{+}=K\left(\omega_{+}\right)$, and $\widehat{Z_{R}}$ satisfies the estimate

$$
\begin{aligned}
\widehat{\widehat{\mid Z_{R}} \mid=} & \mathcal{R}_{2}\left(\omega,|z|+\|f\|_{\infty}\right) \\
& \times\left[\left(\left\|k_{1}\right\|_{\rho}+|z|^{2}+\left\|h_{1}\right\|_{\rho}\right)\left(\left\|k_{1}\right\|_{\rho}+|z|^{2}+\left\|h_{1}\right\|_{\rho}+|z|\left|\omega_{T}-\omega\right|\right)\right. \\
& \left.+|z|\left(\left\|k_{1}\right\|_{\rho}+\left\|h_{1}\right\|_{\rho}\right)\right] \\
= & \mathcal{R}\left(\varepsilon^{1 / 2} \mathbb{M}\right) \frac{\varepsilon^{2} \log (2+\varepsilon t)}{(1+\varepsilon t)^{3 / 2} \sqrt{\varepsilon t}}\left(1+\mathbb{M}^{4}\right) \leqslant \frac{c \varepsilon^{2} \log (2+\varepsilon t)}{(1+\varepsilon t)^{3 / 2} \sqrt{\varepsilon t}} .
\end{aligned}
$$

On the other hand, we have, from (5.2.17) and (5.1.17),

$$
\left|y-\frac{y_{0}}{1+2 \operatorname{Im} K_{+} y_{0} t}\right| \leqslant c\left(\frac{\varepsilon}{1+\varepsilon t}\right)^{3 / 2} \log (2+\varepsilon t)
$$

with $\left|y_{0}-\varepsilon\right| \leqslant c \varepsilon^{3 / 2}$.

With estimate (5.4.17) for $|z|$ and obviously the same one for $\left|z_{1}\right|$, we have

$$
\dot{z}_{1}=i \mu z_{1}+i K_{+} \frac{y_{0}}{1+2 \operatorname{Im} K_{+} y_{0} t} z_{1}+Z_{1}
$$

with

$$
\left|Z_{1}\right| \leqslant c \frac{\varepsilon^{2} \log (2+\varepsilon t)}{(1+\varepsilon t)^{3 / 2} \sqrt{\varepsilon t}} .
$$

Since $y_{0}=\varepsilon+\mathrm{O}\left(\varepsilon^{3 / 2}\right)$, we have that the coefficient $2 \operatorname{Im} K_{+} y_{0}=k_{+} \varepsilon$. We also denote $\delta=\frac{\operatorname{Re} K_{+}}{\operatorname{Im} K_{+}}$. The solution $z_{1}$ of (6.1.4) is written in the form

$$
\begin{aligned}
z_{1} & =\frac{\mathrm{e}^{i \int_{0}^{t} \mu\left(t_{1}\right) d t_{1}}}{\left(1+k_{+} \varepsilon t\right)^{1 / 2-i \delta}}\left[z_{1}(0)+\int_{0}^{t} \mathrm{e}^{-i \int_{0}^{s} \mu\left(t_{1}\right) d t_{1}}\left(1+k_{+} \varepsilon s\right)^{1 / 2-i \delta} Z_{1}(s) d s\right] \\
& =z_{\infty} \frac{\mathrm{e}^{i \int_{0}^{t} \mu\left(t_{1}\right) d t_{1}}}{\left(1+k_{+} \varepsilon t\right)^{1 / 2-i \delta}}+z_{R}
\end{aligned}
$$

where

$$
z_{\infty}=z_{1}(0)+\int_{0}^{\infty} \mathrm{e}^{-i \int_{0}^{s} \mu\left(t_{1}\right) d t_{1}}\left(1+k_{+} \varepsilon s\right)^{1 / 2-i \delta} Z_{1}(s) d s
$$


and

$$
z_{R}=-\int_{t}^{\infty} \mathrm{e}^{i \int_{s}^{t} \mu\left(t_{1}\right) d t_{1}}\left(\frac{1+k_{+} \varepsilon s}{1+k_{+} \varepsilon t}\right)^{1 / 2-i \delta} Z_{1}(s) d s .
$$

For simplicity, we have denoted $\mu\left(t_{1}\right)=\mu\left(\omega\left(t_{1}\right)\right)$ in the above formulas.

It follows from the bound (6.1.5) on $Z_{1}$ that

$$
\left|z_{R}\right| \leqslant c \frac{\varepsilon \log (2+\varepsilon t)}{(1+\varepsilon t)}
$$

Therefore $z_{1}(t)$ satisfies the estimate

$$
z_{1}(t)=z_{\infty} \frac{\exp \left(i \int_{0}^{t} \mu\left(t_{1}\right) d t_{1}\right)}{\left(1+k_{+} \varepsilon t\right)^{\frac{1}{2}-i \delta}}+\mathrm{O}\left(\frac{\varepsilon}{1+\varepsilon t} \log (2+\varepsilon t)\right) .
$$

Here $z_{\infty}=z_{1}(0)+\mathrm{O}(\varepsilon), z=z_{1}+\mathrm{O}\left(\frac{\varepsilon}{1+\varepsilon t}\right)$, and $|z(0)|=\varepsilon^{1 / 2}$. Thus $\left|z_{\infty}\right|=\varepsilon^{1 / 2}+\mathrm{O}(\varepsilon)$. Consequently, the function $z(t)$ satisfies the estimate

$$
z(t)=z_{\infty} \frac{\mathrm{e} i \int_{0}^{t} \mu\left(t_{1}\right) d t_{1}}{\left(1+k_{+} \varepsilon t\right)^{\frac{1}{2}-i \delta}}+\mathrm{O}\left(\frac{\varepsilon}{1+\varepsilon t} \log (2+\varepsilon t)\right) .
$$

From the formulas for $z$, we can easily deduce the asymptotic behavior of $\omega$. Indeed, from (4.1.14) and (5.1.6),

$$
\left|\widehat{\Omega_{R}}\right| \leqslant c\left(\frac{\varepsilon}{1+\varepsilon t}\right)^{2}
$$

Eq. (5.4.16) shows that

$$
\omega_{1}(t)=\omega_{1}(\infty)+\mathrm{O}\left(\frac{\varepsilon}{1+\varepsilon t}\right) .
$$

It follows from (4.1.6) that

$$
\omega(t)=\omega_{+}+\mathrm{O}\left(\frac{\varepsilon}{1+\varepsilon t}\right)
$$

In fact, one could develop a change of variables more precise than (4.1.6) and show that

$$
\omega=\omega_{+}-b_{20}\left(\omega_{+}\right) z^{2}-b_{02}\left(\omega_{+}\right) \bar{z}^{2}+\mathrm{O}\left(\left(\frac{\varepsilon}{1+\varepsilon t}\right)^{3 / 2}\right)
$$

which leads to

$$
\omega=\omega_{+}-\frac{i}{2 \mu_{+}} a\left(\omega_{+}\right)\left(z^{2}-\bar{z}^{2}\right)+\mathrm{O}\left(\left(\frac{\varepsilon}{1+\varepsilon t}\right)^{3 / 2}\right),
$$

where $a(\omega)=\Omega_{20}(\omega)$ is given in (3.2.3) and is purely imaginary. More precisely, 


$$
\begin{aligned}
\omega= & \omega_{+}-\frac{i}{\mu_{+}} \frac{a\left(\omega_{+}\right)}{1+k_{+} \varepsilon t} \\
& \times \operatorname{Re}\left[z_{+}^{2} \mathrm{e}^{2 i \int_{0}^{t} \mu\left(t_{1}\right) d t_{1}} \mathrm{e}^{i \delta} \log \left(1+k_{+} \varepsilon t\right)\right]+\mathrm{O}\left(\left(\frac{\varepsilon}{1+\varepsilon t}\right)^{3 / 2}\right) .
\end{aligned}
$$

In the integral $\int_{0}^{t} \mu\left(t_{1}\right) d t_{1}$, we replace $\mu\left(t_{1}\right)=\mu\left(\omega\left(t_{1}\right)\right)$ by

$$
\mu\left(\omega\left(t_{1}\right)\right)=\mu_{+}+\mu^{\prime}\left(\omega_{+}\right)\left(\omega\left(t_{1}\right)-\omega_{+}\right)+\cdots
$$

where $\mu_{+}=\mu\left(\omega_{+}\right)$. We then see that the integral of the correction converges, although not absolutely. Therefore the dependency of $\omega$ upon $t$ becomes more explicit.

PROPOSITION 6.1. - Under the hypothesis of Theorem 1, the function $\omega(t)$ has the following asymptotic behavior as $t \rightarrow \infty$

$$
\omega \sim \omega_{+}+\frac{b_{+} \varepsilon}{1+k_{+} \varepsilon t} \cos \left(2 \mu_{+} t+b_{1} \log \left(1+k_{+} \varepsilon t\right)+b_{2}\right),
$$

where $b_{+}, b_{1}$ and $b_{2}$ are some constants.

PROPOSITION 6.2. - Under the hypothesis of Theorem 1, the function $z(t)$ has the asymptotic behavior as $t \rightarrow \infty$

$$
z(t) \sim \zeta_{+} \varepsilon^{1 / 2} \frac{\mathrm{e}^{i \mu_{+} t}}{\left(1+k_{+} \varepsilon t\right)^{\frac{1}{2}-i \delta}}, \quad\left|\zeta_{+}\right|=1+O\left(\varepsilon^{1 / 2}\right) .
$$

\subsection{Asymptotic behavior of $\gamma$}

Until now, the function $\gamma$ did not play any essential role in our computations. However, it enters in the main formula for $\psi$ (see (5.4.15)) and therefore, we have to find its asymptotic behavior. In fact, we can repeat the calculation performed for $\omega$ in Section 4.1. The starting point for the representation of $\gamma$ is equation (3.2.1) that we can rewrite

$$
\begin{aligned}
\dot{\gamma}= & \Gamma_{20}(\omega) z^{2}+\Gamma_{11}(\omega) z \bar{z}+\Gamma_{02}(\omega) \bar{z}^{2}+\Gamma_{30}(\omega) z^{3}+\Gamma_{21}(\omega) z^{2} \bar{z}+\Gamma_{12}(\omega) z \bar{z}^{2}+\Gamma_{03} \bar{z}^{3} \\
& +z\left\langle f, \Gamma_{10}^{\prime}\right\rangle+\bar{z}\left\langle f, \Gamma_{01}^{\prime}\right\rangle+\Gamma_{R}
\end{aligned}
$$

and $\Gamma_{R}$ satisfies estimate (3.2.2), which is the same as the one for $\Omega_{R}$. The only difference between the equations for $\omega$ and $\gamma$ is that, in general the coefficient $\Gamma_{11}(\omega) \neq 0$. We can nevertheless perform the same change of variables as for $\omega$ and get

PROPOSITION 6.3. - There exist coefficients $d_{i j}(\omega), 0 \leqslant i, j \leqslant 3$, and vector functions $d_{i j}^{\prime}(x, \omega)$ such that the new function $\gamma_{1}$ defined as

$$
\begin{aligned}
\gamma_{1}= & \gamma+d_{20} z^{2}+d_{02} \bar{z}^{2}+d_{30} z^{3}+d_{21} z^{2} \bar{z}+d_{12} z \bar{z}^{2}+d_{03} \bar{z}^{3} \\
& +z\left\langle f, d_{10}^{\prime}\right\rangle+\bar{z}\left\langle f, d_{01}^{\prime}\right\rangle
\end{aligned}
$$


with $d_{i j}=\bar{d}_{j i}$ is solution of the differential equation

$$
\dot{\gamma}_{1}=\Gamma_{11}(\omega) z \bar{z}+\widehat{\Gamma_{R}}
$$

and $\widehat{\Gamma_{R}}$ satisfies the same estimate (3.2.2) as $\Gamma_{R}$.

Notice that

$$
\Gamma_{11}(\omega)=-\frac{2 E_{2}\left[u, u^{*}\right]}{\left\langle\phi, \phi_{\omega}\right\rangle}
$$

is real. From (6.2.3), it follows that

$$
\dot{\gamma}_{1}=\Gamma_{11}\left(\omega_{+}\right) \frac{\left|z_{+}\right|^{2}}{1+k_{+} \varepsilon t}+\mathrm{O}\left(\left(\frac{\varepsilon}{1+\varepsilon t}\right)^{2}\right)
$$

and

$$
\gamma_{1}(t) \sim \gamma_{+}+\frac{\Gamma_{11}\left(\omega_{+}\right)}{k_{+}} \log \left(1+k_{+} \varepsilon t\right) .
$$

Coming back to the original variable, we get the asymptotic behavior of $\gamma(t)$.

PROPOSITION 6.4. - The long time behavior of the phase factor $\gamma(t)$ is given by

$$
\gamma(t)=\gamma_{+}+c_{+} \log \left(1+k_{+} \varepsilon t\right)+\mathrm{O}\left(\frac{\varepsilon}{1+\varepsilon t}\right) .
$$

\subsection{Asymptotic behavior of $f$}

We finally turn to the function $f$. We know from (5.4.18) and (5.4.19) that in the norms $\|\cdot\|_{\rho}$ and $\|\cdot\|_{\infty}, f$ tends to 0 as $t \rightarrow \infty$. However, this radiative part carries non-zero energy and other integrals of motion, and from this point of view it is not negligible. To control the contribution of $f$ to the integrals of motion, we have to study its asymptotic behavior in the usual $L^{2}$ norm. We recall the representation

$$
f=g+h, \quad g=P_{d}\left(\omega_{+}\right) f, \quad h=P_{c}\left(\omega_{+}\right) f .
$$

Proceeding in the same way as in (4.2.5), we have

$$
\|g\|_{2} \leqslant \mathcal{R}_{1}(\omega)\left|\omega_{+}-\omega\right|\|h\|_{\rho} \leqslant c\left(\frac{\varepsilon}{1+\varepsilon t}\right)^{2},
$$

and therefore,

$$
\|f\|_{2}=\|h\|_{2}+\mathrm{O}\left(\left(\frac{\varepsilon}{1+\varepsilon t}\right)^{2}\right) .
$$

The function $h$ is solution of

$$
\dot{h}=C_{+} h-P_{c}\left(\omega_{+}\right) j E_{2}[w, w]+H_{R} .
$$


Therefore, from

$$
h=\mathrm{e}^{\int_{0}^{t} C_{+}\left(t_{1}\right) d t_{1}}\left(h_{0}+h_{2}+h_{\infty}\right)+\Delta_{2}+\Delta_{\infty}
$$

where

$$
\begin{aligned}
& h_{2}=-\int_{0}^{\infty} d s \mathrm{e}^{-\int_{0}^{s} C_{+}\left(t_{1}\right) d t_{1}} P_{c}\left(\omega_{+}\right) j E_{2}[w, w], \\
& h_{\infty}=\int_{0}^{\infty} d s \mathrm{e}^{-\int_{0}^{s} C_{+}\left(t_{1}\right) d t_{1}} H_{R}(s), \\
& \Delta_{2}=\int_{t}^{\infty} d s \mathrm{e}^{-\int_{t}^{s} C_{+}\left(t_{1}\right) d t_{1}} P_{c}\left(\omega_{+}\right) j E_{2}[w, w], \\
& \Delta_{\infty}=-\int_{t}^{\infty} d s \mathrm{e}^{-\int_{t}^{s} C_{+}\left(t_{1}\right) d t_{1}} H_{R}(s) .
\end{aligned}
$$

From (5.1.11) and (5.1.12), we know that

$$
\left\|H_{R}\right\|_{2} \leqslant c\left(\frac{\varepsilon}{1+\varepsilon t}\right)^{3 / 2} \log (2+\varepsilon t) .
$$

This implies that the integrals (6.3.7) and (6.3.9) converge and, in addition

$$
\left\|\Delta_{\infty}\right\|_{2}=\mathrm{O}\left(\left(\frac{\varepsilon}{1+\varepsilon t}\right)^{1 / 2} \log (2+\varepsilon t)\right) .
$$

The integrals in (6.3.6) and (6.3.8) also converge and define elements of $L^{2}$, although they do not converge absolutely. Similarly to Lemma 5.2, one can prove

Proposition 6.5. - We have $h_{2} \in L^{2}$ and

$$
\Delta_{2}=\mathrm{O}\left(\frac{\varepsilon}{1+\varepsilon t}\right)
$$

Comments on this proposition are given in Appendix A.

Finally, we turn to

$$
f(t)=\mathrm{e}^{\int_{0}^{t} C_{+}\left(t_{1}\right) d t_{1}}\left[h_{0}+h_{2}+h_{\infty}+\mathrm{O}\left(\left(\frac{\varepsilon}{1+\varepsilon t}\right)^{1 / 2} \log (2+\varepsilon t)\right)\right] .
$$

The leading term here can be treated by the methods of scattering theory. Recall that from (5.2.60)

$$
\mathrm{e}^{\int_{0}^{t} C_{+}\left(t_{1}\right) d t_{1}}=\left(\mathrm{e}^{i v} P_{\infty}^{+}+\mathrm{e}^{-i v} P_{\infty}^{-}\right) \mathrm{e}^{C\left(\omega_{+}\right) t}
$$


From (6.1.19), we see that the integral $\int_{0}^{\infty}\left(\omega-\omega_{+}\right) d t_{1}$ converges (not absolutely). Therefore, as $t \rightarrow \infty$,

$$
v(t) \sim \int_{0}^{\infty}\left(\omega-\omega_{+}\right) d t_{1}+\gamma(t)-\gamma(0) .
$$

Without loss of generality, we can suppose that $\gamma(0)=0$. The operator $C\left(\omega_{+}\right)$is not anti-selfadjoint, since $C\left(\omega_{+}\right)=i \mathcal{D}$ and $\mathcal{D} \neq \mathcal{D}^{*}$, because the potential $V$ is complex. Therefore, $\mathrm{e}^{C\left(\omega_{+}\right) t}$ is not a unitary group. Nevertheless,

$$
C\left(\omega_{+}\right)=j^{-1}\left(-\frac{\partial^{2}}{\partial x^{2}}+\omega_{+}\right)+V\left(\omega_{+}, x\right)
$$

and $V$ is exponentially decreasing at infinity. Thus we can apply standard results of spectral theory to $\mathrm{e}^{C\left(\omega_{+}\right) t} \tilde{h}$, where $\tilde{h}=h_{0}+h_{2}+h_{\infty}$.

PROPOSITION 6.6. - We have the asymptotic formula

$$
\mathrm{e}^{C\left(\omega_{+}\right) t} \tilde{h}=\mathrm{e}^{C_{0} t} h_{+}+\mathrm{o}(1)
$$

as $t \rightarrow \infty$, where $h_{+}=W \tilde{h}$ and $W$ is a bounded operator in $L^{2}(\mathbb{R})$, that can be seen as a wave operator: $W$ is the strong limit in $L^{2}$ of $\mathrm{e}^{-C_{0} t} \mathrm{e}^{C\left(\omega_{+}\right) t}$, and

$$
C_{0}=j^{-1}\left(-\frac{\partial^{2}}{\partial x^{2}}+\omega_{+}\right) \text {. }
$$

The proof of this result is standard. We write that

$$
\begin{aligned}
\mathrm{e}^{C\left(\omega_{+}\right) t} f(x)= & \frac{1}{i} \int_{\omega_{+}}^{\infty}\left(\langle f, u(i \lambda)\rangle u(x, i \lambda) \mathrm{e}^{i \lambda t}\right. \\
& \left.+\left\langle f, u^{*}(i \lambda)\right\rangle u^{*}(x, i \lambda)\right) \mathrm{e}^{-i \lambda t} \theta(\lambda) d \lambda .
\end{aligned}
$$

As $t \rightarrow \infty$, the asymptotic behavior of the above integral in $L^{2}$ is determined by the asymptotic behavior of $u(x, i \lambda)$ as $x \rightarrow \infty$ (see (A.4) of Appendix A). The long time behavior is the same as for the group $\mathrm{e}^{j^{-1}\left(L+\omega_{+}\right) t}$.

Proposition 6.7. - We have

$$
f=\mathrm{e}^{j^{-1} v} \mathrm{e}^{C_{0} t} h_{+}+\mathrm{o}(1) .
$$

Proof. - Since $W$ satisfies the standard property

$$
W \varphi\left(C\left(\omega_{+}\right)\right)=\varphi\left(C_{0}\right) W,
$$

we have that

$$
\left(\mathrm{e}^{i v} P_{\infty}^{+}+\mathrm{e}^{-i v} P_{\infty}^{-}\right) \mathrm{e}^{C\left(\omega_{+}\right) t} \tilde{h}=\left(\mathrm{e}^{i v} P_{0}^{+}+\mathrm{e}^{-i v} P_{0}^{-}\right) \mathrm{e}^{C_{0} t} h_{+}+\mathrm{o}(1) .
$$


In the above equation, $P_{0}^{ \pm}$are the projection operators onto the positive/negative imaginary parts of the continuous spectrum of $C_{0}$. It is obvious that

$$
\mathrm{e}^{j^{-1} v}=\mathrm{e}^{i v} P_{0}^{+}+\mathrm{e}^{-i v} P_{0}^{-},
$$

therefore,

$$
\left(\mathrm{e}^{i v} P_{\infty}^{+}+\mathrm{e}^{-i v} P_{\infty}^{-}\right) \mathrm{e}^{C\left(\omega_{+}\right) t} \tilde{h}=\mathrm{e}^{j^{-1} v} \mathrm{e}^{C_{0} t} h_{+}+\mathrm{o}(1) .
$$

Combining the asymptotic formulas for $\omega, \gamma, z$, and $f$, we get

THEOREM 2.-Suppose that the hypothesis of Theorem 1 are satisfied. Then for $\varepsilon$ small enough the solution of the nonlinear Schrödinger equation (1.0.5) have the following behavior as $t \rightarrow \infty$

$$
\begin{aligned}
\psi(x, t)= & \mathrm{e}^{j\left(\omega_{+} t+\gamma_{+}(t)+\chi\right)}\left[\phi\left(x, \omega_{+}\right)+z_{+}(t) u\left(x, \omega_{+}\right)+\bar{z}_{+}(t) u^{*}\left(x, \omega_{+}\right)\right] \\
& +\mathrm{e}^{j^{-1} L t} h_{+}+\mathrm{o}(1)
\end{aligned}
$$

in $L_{2}$, where

$$
\varkappa=\int_{0}^{\infty}\left(\omega\left(t_{1}\right)-\omega_{+}\right) d t_{1}+\gamma_{\infty}, \quad L=-\frac{\partial^{2}}{\partial x^{2}} .
$$

The operator $L$ is self-adjoint in $L^{2}$ with its usual domain.

This result can be rewritten in terms of the original complex notation. As $t \rightarrow \infty$, the solution of the nonlinear Schrödinger equation behaves as follows

$$
\begin{aligned}
\psi= & \mathrm{e}^{i\left(\omega_{+} t+\gamma_{+}(t)+\varkappa\right)}\left[\varphi\left(x, \omega_{+}\right)+z_{+}(t) v_{+}\left(x, \omega_{+}\right)+\bar{z}_{+}(t) v_{-}\left(x, \omega_{+}\right)\right] \\
& +\mathrm{e}^{-i L t} h_{+}+\mathrm{o}(1)
\end{aligned}
$$

with $v_{ \pm}(x, \omega)=u_{1}(x, \omega) \pm i u_{2}(x, \omega)$ and $h_{+}=\left(h_{+}\right)_{1}+i\left(h_{+}\right)_{2}$.

In conclusion, let us compute the classical integrals of motion for the nonlinear Schrödinger equation in terms of the limiting parameters $\omega_{+}$and $h_{+}$. We will restrict the calculation to the conservation of the $L^{2}$-norm. The conservation of the energy is treated similarly. We have

$$
\left\|\psi_{0}\right\|_{2}^{2}=\|\psi(\cdot, t)\|_{2}^{2}=\|\phi(\cdot, \omega)+w(\cdot, t, \omega)+f(\cdot, t)\|_{2}^{2}
$$

with $w(x, t, \omega)=z(t) u(x, \omega)+\bar{z}(t) u^{*}(x, \omega)$. We compute the limit of the right-hand side as $t \rightarrow \infty$. We can replace $\phi(\cdot, \omega)$ by $\phi\left(\cdot, \omega_{+}\right), w(\cdot, t, \omega)$ by $w\left(\cdot, t, \omega_{+}\right)$and $f$ by $\mathrm{e}^{-j\left(\omega_{+} t+\gamma_{+}+\delta_{+}\right)} \mathrm{e}^{j^{-1} L\left(\omega_{+}\right) t} h_{+}$. It is clear that

$$
\begin{aligned}
& \left\|\phi\left(\cdot, \omega_{+}\right)+w\left(\cdot, t, \omega_{+}\right)+f\right\|_{2}^{2} \\
& \quad=\|\phi\|_{2}^{2}+\|w\|_{2}^{2}+\|f\|_{2}^{2}+2\langle\phi, f\rangle+2\langle\phi, w\rangle+2\langle w, f\rangle .
\end{aligned}
$$

When $t \rightarrow \infty,\|w\|_{2} \rightarrow 0$, and $\|f\|_{2}^{2}=\left\|h_{+}\right\|_{2}^{2}$. Also,

$$
\begin{aligned}
& |\langle\phi, w\rangle| \leqslant\|\phi\|_{2}\|w\|_{2}, \quad|\langle\phi, f\rangle| \leqslant\left\|\rho^{-1} \phi\right\|_{2}\|f\|_{\rho} \rightarrow 0, \\
& |\langle w, f\rangle| \leqslant\left\|\rho^{-1} w\right\|_{2}\|f\|_{\rho} \leqslant c|z|\left\|\rho^{-1} u\left(\cdot, \omega_{+}\right)\right\|_{2}\|f\|_{\rho} \rightarrow 0 .
\end{aligned}
$$


Therefore, $\|\phi+w+f\|_{2}^{2} \rightarrow\left\|\phi\left(\cdot, \omega_{+}\right)\right\|_{2}+\left\|h_{+}\right\|_{2}^{2}$, and finally,

$$
\|\psi(\cdot, t)\|_{2}^{2}=\|\psi(\cdot, 0)\|_{2}^{2}=\left\|\phi\left(\cdot, \omega_{+}\right)\right\|_{2}^{2}+\left\|h_{+}\right\|_{2}^{2} .
$$

\section{Acknowledgements}

V.B. acknowledges support from INTAS grant 97-10812. V.B. thanks the Department of Mathematics at the University of Toronto for its support and hospitality during the spring semesters of 2000 and 2001. C.S. acknowledges support from NSERC operating grant OGP0046179.

\section{Appendix A. Properties of eigenfunctions $u(x, \lambda)$}

In this appendix, we recall some analytical properties of the spectral resolution (2.1.13). Some of these properties were described in detail in [2] where this decomposition was justified, some of them were discussed in [3]. Here, we give some additional comments, and for this purpose, it is necessary to state more precisely the properties of $u(x, \lambda)$. The results in [2] were obtained in terms of an operator $H$ related to $C$ by the similarity $C=i U H U^{-1}$ where $U$ is a constant $2 \times 2$ matrix. They were developed in a more general context where the authors did not restrict $C$ to even functions. In that case, the dimension of the invariant subspace corresponding to $\lambda=0$ is, at least, 4 , and the multiplicity of the continuous spectrum is equal to 2 .

Due to the exponential decay of the potential $V$ at infinity, the properties of the eigenfunctions of $C$ are very close to the properties of simple exponentials and the resolution is similar to the Fourier transform.

First, the solutions of the unperturbed equation

$$
C_{0} u=i \lambda u, \quad C_{0}=j^{-1}\left(-\partial_{x x}+\omega\right),
$$

are

$$
\begin{array}{ll}
f_{1}=\mathrm{e}^{i k_{1} x} v_{1}, & f_{2}=\mathrm{e}^{-i k_{1} x} v_{1}, \\
g_{1}=\mathrm{e}^{i k_{2} x} v_{2}, & g_{2}=\mathrm{e}^{-i k_{2} x} v_{2},
\end{array}
$$

where $k_{1}=\sqrt{\lambda-\omega}, k_{2}=i \sqrt{\lambda+\omega}, v_{1}=\left(\begin{array}{l}1 \\ i\end{array}\right), v_{2}=\left(\begin{array}{c}1 \\ -i\end{array}\right)$. In this case, (2.1.13) is nothing else but the classical cos-Fourier transform for even functions $f: \mathbb{R} \rightarrow \mathbb{C}^{2}$.

The basis in $\mathbb{C}^{2}$ is chosen from the eigenvectors of $j$ :

$$
\begin{array}{ll}
u(x, \lambda)=\cos \left(k_{1} x\right) v_{1} & \text { and } j v_{1}=-i v_{1}, \\
u(x, \lambda)=\cos \left(k_{2} x\right) v_{2} & \text { and } j v_{2}=i v_{2} .
\end{array}
$$

In [2], it was shown (it is a general fact) that the perturbed equation

$$
C u=i \lambda u, \quad C=C_{0}+V, \quad\|V(x, \omega)\| \leqslant k(\omega) \mathrm{e}^{-\gamma|x|}, \quad \gamma=2 \sqrt{\omega} r,
$$


has a solution $g_{1}^{+}\left(x, k_{1}\right)$ that is uniquely defined on the set $\mathbb{R} \times D$ where $D=$ $\left\{k_{1}:\left|\operatorname{Im}\left(k_{1}\right)\right| \leqslant \gamma_{1}\right\}, \gamma_{1}<\gamma$ by the asymptotic condition

$$
g_{1}^{+} \sim g_{1}, \quad x \rightarrow+\infty .
$$

This solution is also an analytic function of $\lambda$, including the point $\lambda=\omega$. More precisely, $g_{1}^{+}$is analytic on a suitable parabolic vicinity of the semi-axis $\lambda \geqslant \omega$.

The second solution $f_{1}^{+}$is rapidly decreasing as $x \rightarrow+\infty$ (modulo the solution $g_{1}^{+}$) if $\operatorname{Im} k_{1}>0$. It is also defined on $D$ as an analytic function of $\lambda$ and can be written

$$
f_{1}^{+}\left(x, k_{1}\right)=\mathrm{e}^{i k_{1} x}\left[v_{1}+R_{1}\right]+R_{2},
$$

with uniform estimates on any set $[a, \infty) \times D, a$ is fixed, in the form:

$$
\left|R_{1}\right| \leqslant \operatorname{const}\left(1+\left|k_{1}\right|\right)^{-1} \mathrm{e}^{-\gamma x}, \quad\left|R_{2}\right| \leqslant \operatorname{const}\left(1+\left|k_{1}\right|\right)^{-1}\left|\mathrm{e}^{i k_{2} x}\right| .
$$

In general, this solution is not analytic at $\lambda=\omega$, and is analytic on a double-sheet Riemann surface of the function $k_{1}=\sqrt{\lambda-\omega}$. It is convenient to see the semi-axis as the cut on this surface.

Finally, there exists an analogous solution $f_{2}^{+}\left(x, k_{1}\right)=f_{1}^{+}\left(x,-k_{1}\right)$ that satisfies a similar estimate:

$$
f_{2}^{+}\left(x, k_{1}\right)=\mathrm{e}^{-i k_{1} x}\left[v_{1}+R_{3}\right]+R_{4},
$$

again on $[a, \infty) \times D$, with

$$
\left|R_{3}\right| \leqslant \operatorname{const}\left(1+\left|k_{1}\right|\right)^{-1} \mathrm{e}^{-\gamma x}, \quad\left|R_{4}\right| \leqslant \operatorname{const}\left(1+\left|k_{1}\right|\right)^{-1}\left|\mathrm{e}^{i k_{2} x}\right| .
$$

As already mentioned, $f_{1}^{+}$is defined modulo $g_{1}^{+}$. In general, the solution $f_{1}^{+}$grows when $x \rightarrow-\infty$ like $g_{1}$. But there exists a choice of $f_{1}^{+}$such that, for $\lambda>\omega$, it remains bounded as $x \rightarrow-\infty$. Such a solution is defined uniquely and was referred to in [2] as the solution of the scattering problem. Let us denote it by $\mathfrak{F}\left(x, k_{1}\right)$. It is easy to see that

$$
\mathfrak{F}^{*}\left(x,-k_{1}^{*}\right)=\sigma_{3} \mathfrak{F}\left(x, k_{1}\right), \quad \sigma_{3}=\left(\begin{array}{cc}
1 & 0 \\
0 & -1
\end{array}\right),
$$

where $*$ denotes the complex conjugation.

For $x \geqslant a$, this solution has the same properties as the general function $f_{1}^{+}$defined in (A.5):

$$
\mathfrak{F}(x, \lambda)=\mathrm{e}^{i k_{1} x}\left[v_{1}+R_{1}\right]+R_{2} .
$$

As for $x \leqslant a$, its behavior is a little more complicated:

$$
\mathfrak{F}(x, \lambda)=\mathrm{e}^{i k_{1} x}\left[b\left(k_{1}\right) v_{1}+R_{5}\right]+\mathrm{e}^{-i k_{1} x}\left[c\left(k_{1}\right) v_{1}+R_{6}\right]+R_{7},
$$

with uniform estimates on any set $(-\infty, a] \times D$ :

$$
\begin{gathered}
\left|R_{5}\right| \leqslant \operatorname{const}\left(1+\left|k_{1}\right|\right)^{-1} \mathrm{e}^{-\gamma x}, \quad\left|R_{6}\right| \leqslant \operatorname{const}\left(1+\left|k_{1}\right|\right)^{-1} \mathrm{e}^{-\gamma x}, \\
\left|R_{7}\right| \leqslant \operatorname{const}\left(1+\left|k_{1}\right|\right)^{-1}\left|\mathrm{e}^{-i k_{2} x}\right| .
\end{gathered}
$$


The coefficients $b$ and $c$ satisfies the equalities $b(k)=b^{*}\left(-k_{1}^{*}\right), c(k)=c^{*}\left(-k_{1}^{*}\right)$, and for large $k_{1}$, have the asymptotic expansions:

$$
b \sim 1+\frac{b_{1}}{k_{1}}+\cdots, \quad c \sim \frac{c_{1}}{k_{1}}+\cdots .
$$

We connect now $\mathfrak{F}$ with $u$ from (2.1.12). The potential $V$ is even and we can consider an even solution with respect to $x$ and $k_{1}$

$$
u_{0}=\mathfrak{F}\left(x, k_{1}\right)+\mathfrak{F}\left(-x, k_{1}\right)
$$

of (A.3). It is bounded for all $x \in \mathbb{R}$ if $\lambda>\omega$. As $x \rightarrow+\infty$, up to exponentially decreasing terms

$$
u_{0} \sim\left[\mathrm{e}^{i k_{1} x}\left(1+c\left(k_{1}\right)\right)+\mathrm{e}^{-i k_{1} x} b\left(k_{1}\right)\right] v_{1} .
$$

From this asymptotic behavior it follows, in particular, that

$$
u_{0}=\left(1+c\left(k_{1}\right)\right) f_{1}^{+}\left(x, k_{1}\right)+b\left(k_{1}\right) f_{1}^{+}\left(x,-k_{1}\right) .
$$

The first vector component of this solution is asymptotically

$$
\left(u_{0}\right)_{1} \sim \mathrm{e}^{i k_{1} x}(1+c)+\mathrm{e}^{-i k_{1} x} b .
$$

Up to a constant factor, $\left(u_{0}\right)_{1}$ must be real, therefore $|1+c|=|b|$. If the semi-axis $\lambda>\omega$ does not contain embedded points of the point spectrum of $C$, then $b\left(k_{1}\right) \neq 0$ for such $\lambda$ [2]. This function can have roots in $D$ with $\operatorname{Im}\left(k_{1}\right)<0$, but their number if finite. Therefore, there exists such a strip $D$ that is free of roots of $b$.

Now for $u(\cdot, \lambda)$, one can take the function:

$$
\begin{aligned}
& u\left(x, k_{1}\right)=[b(1+c)]^{-1 / 2} u_{0}=M^{-1}\left(k_{1}\right) f_{1}^{+}\left(x, k_{1}\right)+M\left(k_{1}\right) f_{1}^{+}\left(x,-k_{1}\right), \\
& M=\left(\frac{b}{1+c}\right)^{1 / 2}, \quad M\left(-k_{1}\right)=M^{*}\left(k_{1}^{*}\right) .
\end{aligned}
$$

Notice that $u(x, \lambda)$ and $u\left(x, k_{1}\right)$ are the same objects seen as functions of different but related variables.

It is clear that $|M|=1$, therefore $M\left(k_{1}\right) M\left(-k_{1}\right)=1$, and

$$
u\left(x, k_{1}\right)=M\left(-k_{1}\right) f_{1}^{+}\left(x, k_{1}\right)+M\left(k_{1}\right) f_{1}^{+}\left(x,-k_{1}\right) .
$$

Its first component has the asymptotic behavior

$$
u_{1} \sim \mathrm{e}^{i k_{1} x} M\left(-k_{1}\right)+\mathrm{e}^{-i k_{1} x} M\left(k_{1}\right)=2 \cos \left(k_{1} x-\vartheta\right),
$$

where for real $k_{1}, M=\mathrm{e}^{i \vartheta}$. When $k_{1} \rightarrow \infty, M\left(k_{1}\right) \rightarrow 1$.

Up to exponentially decreasing terms, we have that, as $x \rightarrow \infty, j u \sim-i u$. Similarly, $j u^{*} \sim i u^{*}$.

Let us emphasize three important properties of the solution $u$ : (1) the representations (A.9) and (A.10) are satisfied for complex $k_{1}$ in the domain $D$; (2) these representations 
can be differentiated with respect to both variables $x$ and $k_{1}$ any number of times; (3) since $\lambda=\omega$ is not a virtual level (i.e., there are no solutions bounded with respect to $x$ at this point), $u(x, 0)=0$. In particular, $\vartheta(0)=\pi(\bmod 2 \pi)$.

Coming back to the expansion formula (2.1.13), consider the first term of the expansion

$$
P_{+} f(x)=\frac{1}{4 \pi i} \int_{\omega}^{\infty} u\left(x, k_{1}\right)\left\langle f, j u\left(\cdot, k_{1}\right)\right\rangle \frac{d \lambda}{2 \sqrt{\lambda-\omega}} .
$$

Due to (A.19), this obviously can be transformed into

$$
P_{+} f(x)=\frac{1}{4 \pi i} \int_{-\infty}^{+\infty} M\left(-k_{1}\right) f_{1}^{+}\left(x, k_{1}\right)\left\langle f, j u\left(\cdot, k_{1}\right)\right\rangle d k_{1} .
$$

Analogous formulas can be obtained for $P_{-} f$. Notice that they are quite convenient for the representation of $P_{+} f$ and $P_{-} f$ for positive $x$. It is sufficient for our purposes.

We conclude the appendix with some additional remarks on Proposition 2.1 and Lemmas 5.1 and 5.2.

\section{A.1. Comments on Proposition 2.1}

From (2.1.13), we have

$$
\begin{aligned}
P_{c} j^{-1} f-i\left(P_{+}-P_{-}\right) f= & \frac{1}{i} \int_{\omega}^{\infty}[u(\cdot, i \lambda)\langle f, j(j u+i u)(\cdot, i \lambda)\rangle \\
& \left.+u^{*}(\cdot, i \lambda)\left\langle f, j\left(j u^{*}-i u^{*}\right)(\cdot, i \lambda)\right\rangle\right] \theta(\lambda) d \lambda .
\end{aligned}
$$

Let us recall that on some strip around the real axis $k_{1}$

$$
|j u+i u| \leqslant \operatorname{const}\left(1+\left|k_{1}\right|\right)^{-1} \mathrm{e}^{-\gamma^{\prime \prime} x}, \quad \gamma^{\prime \prime}>0,
$$

and a similar bound for $j u^{*}-i u^{*}$. Therefore, the second entries in the Fourier coefficients $\langle f, \cdot\rangle$ are exponentially decreasing and the coefficients can be analytically continued, their analytic continuation remaining bounded on this strip by const $(1+$ $\left.\left|k_{1}\right|\right)^{-1}\|f\|_{\rho}$ with any weight function $\rho=\left(1+x^{2}\right)^{-\alpha}, \alpha>0$.

Consider, for example, the first term in the right-hand-side of (A.23). We transform it to a form similar to (A.22) and shift the contour of integration to the straight line $\operatorname{Im}\left(k_{1}\right)=\kappa$, taking $\kappa$ positive small. The function $f_{2}^{+}$for $x>0$ contains, on the contour of integration, a main term and a remainder uniformly exponentially decreasing for large $x$ and decreasing as $k_{1}^{-1}$ for large $k_{1}$, see (A.5)-(A.6). The contribution of the remainder as a function of $x$ can be estimated in $L^{2}$-norm with any growing power weight. The contribution of the main term is completely similar to the standard Fourier integral along the same straight line. In particular, it contains the exponentially decreasing at $+\infty$ in $x$ factor, and up to this factor is the standard Fourier-transform on the real axis. Using that the Fourier coefficients are $L^{2}$-functions of $k_{1}$, we immediately 
estimate the $L^{2}$-norm of this contribution with any growing power weight. It is enough to have the estimates for positive $x$. The second term in the right-hand-side of (A.23) is treated similarly.

\section{A.2. Comments on Lemma 5.2}

The proof of (5.2.40) is a simple modification of that of Lemma 5.1. Indeed, when using spectral resolutions, the operator $C_{T}^{-1}$ contributes to integral (2.1.12) as a factor $\lambda^{-1}$ which is not singular on the contour of integration.

Notice that we will use this estimate for functions $\alpha$ that are exponentially decreasing at infinity. Therefore, when expressing the left side of (5.2.41), we can use the spectral representation and the analyticity of Fourier coefficients. Due to the analyticity, we can shift the part of the contour of integration close to the singular point from the real axis to the corresponding semi-plane as a semi-circle where $\mathrm{e}^{i \lambda t}, t \geqslant 0$, remains bounded, and avoid singularities at $\lambda= \pm i \mu$. We can then easily repeat the proof of Lemma 5.1.

In the case of a general function $\alpha$, we have to complement the previous analysis with some additional technique. The condition $\left\|\left(1+x^{2}\right)^{3 / 2} \alpha\right\|_{1}<\infty$ implies, that the Fourier coefficients of $\alpha$, for example, $\widehat{\alpha}(i \lambda)=\langle\alpha, j u(\cdot, i \lambda)\rangle$ have, as in the case of Lemma 5.1 additional continuous derivatives with respect to $\lambda$. Consider the function

$$
\mathrm{e}^{C_{T} t}\left(C_{T} \pm 2 i \mu-0\right)^{-1} P_{T}^{+} \alpha=\frac{1}{i} \int_{\omega_{T}}^{\infty} \mathrm{e}^{i \lambda t} \frac{1}{i \lambda-2 i \mu-0} \widehat{\alpha}(i \lambda) u(x, i \lambda) \theta(\lambda) d \lambda .
$$

We restrict to functions $\alpha$ satisfying the condition $P_{T}^{+} \alpha=\alpha$. The case $P_{T}^{-} \alpha=\alpha$ can be treated similarly. Let $\chi(\lambda)$ be a cut-off function with support in a neighborhood of 0 , which is analytic near 0 . We write $\widehat{\alpha}(i \lambda)=\widehat{\alpha}_{1}(i \lambda)+\widehat{\alpha}_{2}(i \lambda)$ with

$$
\widehat{\alpha}_{1}(i \lambda)=(\widehat{\alpha}(i \lambda)-\widehat{\alpha}(2 i \mu)) \chi(\lambda-2 \mu), \quad \widehat{\alpha}_{2}(i \lambda)=\widehat{\alpha}(2 i \mu) \chi(\lambda-2 \mu) .
$$

The contribution of $\widehat{\alpha}_{1}(i \lambda)$ to the integral (A.25) is not singular and can be estimated with the help of (5.2.39). To avoid the singularity in the contribution of $\widehat{\alpha}_{2}(i \lambda)$, we again deform the contour of integration in a small vicinity of $\lambda=2 \mu$, keeping $\mathrm{e}^{i \lambda t}$ bounded for $t \geqslant 0$. This completes the comments on Lemma 5.2.

\section{A.3. Comments on Proposition 6.5}

In Proposition 6.5, we deal with two integrals

$$
h_{2}=-\int_{0}^{\infty} d s \mathrm{e}^{-\int_{0}^{s} C_{+}\left(t_{1}\right) d t_{1}} P_{c}\left(\omega_{+}\right) j E_{2}[w, w]
$$

and

$$
\Delta_{2}=-\int_{t}^{\infty} d s \mathrm{e}^{-\int_{t}^{s} C_{+}\left(t_{1}\right) d t_{1}} P_{c}\left(\omega_{+}\right) j E_{2}[w, w] .
$$


The proof of this proposition is close to that of Lemma 5.2. Recall first that

$$
E_{2}[w, w]=z^{2} E_{2}[u, u]+2 z \bar{z} E_{2}\left[u, u^{*}\right]+\bar{z}^{2} E_{2}\left[u^{*}, u^{*}\right] .
$$

To find the leading order of $h_{2}$ and $\Delta_{2}$, it is enough to replace $z$ by its leading asymptotic term (6.1.20). The correction terms to (6.1.20) lead to corrections terms for $h_{2}$ and $\Delta_{2}$.

After substitution of (A.29) into (A.27), the contribution of $|z|^{2}$ has the form

$$
\begin{aligned}
h_{2}^{(1,1)} & \sim-\int_{0}^{\infty} d s \mathrm{e}^{-\int_{0}^{s} C_{+}\left(t_{1}\right) d t_{1}} \frac{\varepsilon\left|\zeta_{+}\right|^{2}}{1+\varepsilon s} P_{c}\left(\omega_{+}\right) j E_{2}\left[u, u^{*}\right] \\
& \sim-\int_{0}^{\infty} d s\left(\mathrm{e}^{-i \nu(s)} P_{\infty}^{+}+\mathrm{e}^{i \nu(s)} P_{\infty}^{-}\right) \mathrm{e}^{-C\left(\omega_{+}\right) s} \frac{\varepsilon\left|\zeta_{+}\right|^{2}}{1+\varepsilon s} P_{c}\left(\omega_{+}\right) j E_{2}\left[u, u^{*}\right] .
\end{aligned}
$$

Integration by parts gives

$$
\begin{aligned}
h_{2}^{(1,1)} \sim & -\left(\mathrm{e}^{-i v(0)} P_{\infty}^{+}+\mathrm{e}^{i v(0)} P_{\infty}^{-}\right) \varepsilon\left|\zeta_{+}\right|^{2} C^{-1}\left(\omega_{+}\right) P_{c}\left(\omega_{+}\right) j E_{2}\left[u, u^{*}\right] \\
& -\int_{0}^{\infty} d s \varepsilon\left|\zeta_{+}\right|^{2} C^{-1}\left(\omega_{+}\right) \mathrm{e}^{-C\left(\omega_{+}\right) s} \\
& \times \frac{\partial}{\partial s}\left[\left(\mathrm{e}^{-i \nu(0)} P_{\infty}^{+}+\mathrm{e}^{i v(0)} P_{\infty}^{-}\right) \frac{1}{1+\varepsilon s}\right] P_{c}\left(\omega_{+}\right) j E_{2}\left[u, u^{*}\right] .
\end{aligned}
$$

The projections $P_{\infty}^{ \pm}$are bounded in $L^{2}$, and $C^{-1}\left(\omega_{+}\right)$is bounded in $P_{c}\left(\omega_{+}\right) L^{2}$. Therefore the first term of $h_{2}^{(1,1)}$ belongs to $L^{2}$. The second term in $h_{2}^{(1,1)}$ is an absolutely converging integral in $L^{2}$-norm since $\mathrm{e}^{-C\left(\omega_{+}\right) s}$ is uniformly bounded in $s \in \mathbb{R}$ (see Lemma 5.1). Thus $h_{2}^{(1,1)} \in L^{2}$.

The contributions $h_{2}^{(2,0)}$ and $h_{2}^{(0,2)}$ coming from the terms $z^{2}$ and $\bar{z}^{2}$ of $E_{2}[w, w$,$] can$ be treated similarly with some additions used in Lemma 5.2. Consider, for example,

$$
\begin{aligned}
h_{2}^{(2,0)} \sim & -\int_{0}^{\infty} d s \mathrm{e}^{-\int_{0}^{s} C_{+}\left(t_{1}\right) d t_{1}} \frac{\varepsilon\left|\zeta_{+}\right|^{2} \mathrm{e}^{2 i \mu_{+} s}}{\left(1+k_{+} \varepsilon s\right)^{1-2 i}} P_{c}\left(\omega_{+}\right) j E_{2}[u, u] \\
\sim & -\left(\mathrm{e}^{-i v(0)} P_{\infty}^{+}+\mathrm{e}^{i v(0)} P_{\infty}^{-}\right) \varepsilon \zeta_{+}^{2}\left(C\left(\omega_{+}\right)-2 i \mu_{+}-0\right)^{-1} P_{c}\left(\omega_{+}\right) j E_{2}[u, u] \\
& -\int_{0}^{\infty} d s \varepsilon \zeta_{+}^{2}\left(C\left(\omega_{+}\right)-2 i \mu_{+}-0\right)^{-1} \mathrm{e}^{-\left(C\left(\omega_{+}\right)-2 i \mu_{T}\right) s} \\
& \times \frac{\partial}{\partial s}\left[\left(\mathrm{e}^{-i v(0)} P_{\infty}^{+}+\mathrm{e}^{i v(0)} P_{\infty}^{-}\right) \frac{1}{\left(1+k_{+} \varepsilon s\right)^{1-2 i}}\right] P_{c}\left(\omega_{+}\right) j E_{2}[u, u] .
\end{aligned}
$$

The necessary estimate for $h_{2}^{(2,0)}$ follows from the fact that

$$
\left(C\left(\omega_{+}\right)-2 i \mu_{+}-0\right)^{-1} \mathrm{e}^{-\left(C\left(\omega_{+}\right)-2 i \mu_{T}\right) s} P_{c}\left(\omega_{+}\right) j E_{2}[u, u]
$$

belongs to $L^{2}$ and is estimated uniformly for $s \geqslant 0$. For the latter result, we apply the spectral resolution, and using the properties of $P_{c}\left(\omega_{+}\right) j E_{2}[u, u]$, deform the contour of integration as it is done for the proof of Lemma 5.2. 
As for the integral appearing in $\Delta_{2}$, it is treated similarly. After integration by parts, we get representations similar to (A.31) and (A.32). More precisely, the first term in formulas for $\Delta_{2}$ analogous to (A.31) and (A.32) will contain the decreasing weight $\left(1+k_{+} t\right)^{-1}$ and the second term (which is an integral) will have the weight $\left(1+k_{+} s\right)^{-2}$, leading to absolutely convergent integrals. This ends the comments on Proposition 6.5.

\section{REFERENCES}

[1] T. Cazenave, P.L. Lions, Orbital stability of standing waves for some nonlinear Schrödinger equations, Comm. Math. Phys. 85 (1982) 549-561.

[2] V. Buslaev, G. Perelman, Scattering for the nonlinear Schrödinger equation: states close to a soliton, St. Petersburg Math. J. 4 (1993) 1111-1142.

[3] V. Buslaev, G. Perelman, On the stability of solitary waves for nonlinear Schrödinger equations, Amer. Math. Soc. Transl. 164 (1995) 75-98.

[4] S. Cuccagna, Stabilization of solutions to nonlinear Schrödinger equations, Comm. Pure Appl. Math. LIV (2001) 1110-1145.

[5] P. Deift, X. Zhou, Perturbation theory for infinite dimensional integrable systems on the line, Preprint.

[6] J. Ginibre, G. Velo, On a class of Schrödinger equations. I: The Cauchy problem, General case; II: Scattering theory, General case, J. Funct. Anal. 32 (1979) 1-32, 33-71.

[7] V.E. Grikurov, Perturbation of unstable solitons for generalized NLS with saturating nonlinearity, in: Intern. Seminar 'Day on Diffraction-97', 1997, pp. 170-179.

[8] M. Grillakis, J. Shatah, W. Strauss, Stability theory of solitary waves in the presence of symmetry, Part I, J. Funct. Anal. 74 (1987) 160-197;

M. Grillakis, J. Shatah, W. Strauss, Stability theory of solitary waves in the presence of symmetry, Part II, J. Funct. Anal. 94 (1990) 308-348.

[9] H. McKean, J. Shatah, The nonlinear Schrödinger equation and the nonlinear heat equation reduction to linear form, Comm. Pure Appl. Math. 44 (1991) 1067-1083.

[10] G. Perelman, On the formation of singularities in solutions of the critical nonlinear Schrödinger equation, Ann. Inst. Henri Poincaré 2 (2001) 605-673.

[11] D. Pelinovsky, Yu. Kivshar, V.V. Afanasjev, Internal modes of envelope solitons, Phys. D 116 (1998) 121-142.

[12] A. Soffer, M. Weinstein, Multichannel nonlinear scattering for non-integrable equations, Comm. Math. Phys. 133 (1990) 119-146;

A. Soffer, M. Weinstein, The case of anisotropic potentials and data, J. Differential Equations 98 (1992) 376-390.

[13] A. Soffer, M. Weinstein, Resonances, radiation damping and instability in Hamiltonian nonlinear wave equations, Invent. Math. 136 (1999) 9-74.

[14] W. Strauss, Nonlinear scattering theory at low energy, J. Funct. Anal. 41 (1981) 110-133; J. Funct. Anal. 43 (1981) 281-293.

[15] C. Sulem, P.-L. Sulem, The Nonlinear Schrödinger Equation: Self-focusing and Wave Collapse, in: Applied Mathematical Sciences, Vol. 139, Springer.

[16] M. Weinstein, Lyapunov stability of ground states of nonlinear dispersive evolution equations, Comm. Pure Appl. Math. 39 (1986) 51-68.

[17] H.-T. Yau, T.-P. Tsai, Asymptotic dynamics of nonlinear Schrödinger equations: resonance dominated and radiation dominated solutions, Comm. Pure Appl. Math. LV (2002) 1-64. 